\title{
Experimental studies on new therapeutic approaches in atrial fibrillation
}

Citation for published version (APA):

Linz, D. K. (2013). Experimental studies on new therapeutic approaches in atrial fibrillation. [Doctoral Thesis, Maastricht University]. Maastricht University. https://doi.org/10.26481/dis.20131114dl

Document status and date:

Published: 01/01/2013

DOI:

10.26481/dis.20131114dl

Document Version:

Publisher's PDF, also known as Version of record

\section{Please check the document version of this publication:}

- A submitted manuscript is the version of the article upon submission and before peer-review. There can be important differences between the submitted version and the official published version of record.

People interested in the research are advised to contact the author for the final version of the publication, or visit the DOI to the publisher's website.

- The final author version and the galley proof are versions of the publication after peer review.

- The final published version features the final layout of the paper including the volume, issue and page numbers.

Link to publication

\footnotetext{
General rights rights.

- You may freely distribute the URL identifying the publication in the public portal. please follow below link for the End User Agreement:

www.umlib.nl/taverne-license

Take down policy

If you believe that this document breaches copyright please contact us at:

repository@maastrichtuniversity.nl

providing details and we will investigate your claim.
}

Copyright and moral rights for the publications made accessible in the public portal are retained by the authors and/or other copyright owners and it is a condition of accessing publications that users recognise and abide by the legal requirements associated with these

- Users may download and print one copy of any publication from the public portal for the purpose of private study or research.

- You may not further distribute the material or use it for any profit-making activity or commercial gain

If the publication is distributed under the terms of Article $25 \mathrm{fa}$ of the Dutch Copyright Act, indicated by the "Taverne" license above, 


\title{
Experimental Studies on New Therapeutic
}

\section{Approaches in Atrial Fibrillation}

\author{
Proefschrift
}

ter verkrijging van de graad van doctor aan de Universiteit Maastricht, op gezag van de Rector Magnificus,

Prof. dr. L. L. G Soete

volgens het besluit van de College van Decanen, in het openbaar te verdedingen

op donderdag 14 November 2013 om 16.00 uur

door

\section{Dominik Linz}


Supervisors: Prof. dr. Ulrich Schotten

Prof. dr. Michael Böhm

Co-supervisor: Dr. Sander Verheule

Assessment Committee:

Prof. dr. Frits Prinzen (Chairman)

Prof. dr. Isabelle van Gelder, UMC Groningen

Prof. dr. Harry Crijns

Prof. dr. Thomas Unger

Dr. Klaus Wirth, Frankfurt am Main, D 


\section{Contents}

Chapter 1: General Introduction

Chapter 2: CathepsinA-Inhibitor Reduces Susceptibility to Atrial Tachyarrhythmia and Attenuates Changes in Atrial Emptying Function in Old Zucker Diabetic Rats.

Chapter 3: Negative Tracheal Pressure During Obstructive Respiratory Events Promotes Atrial Fibrillation by Vagal Activation.

Chapter 4: Combined Blockade of Early And Late Activated Atrial Potassium Currents Suppresses Atrial Fibrillation in a Pig Model of Obstructive Apnea.

Chapter 5: Renal Sympathetic Denervation Suppresses AF-Inducibility in a Pig Model For Obstructive Sleep Apnea.

Chapter 6: Zooming in on the Focus: High Density Mapping of the Interaction between Ectopy and Re-entry during Atrial Fibrillation

Chapter 7: General Discussion

\section{Summery}

Acknowledgment

Curriculum Vitae and Publications 
Chapter 1:

General Introduction 


\section{General Introduction}

$1 \%$ of the European population suffers from atrial fibrillation (AF) and the number of AF-patients is expected to double or triple within the next two to three decades. ${ }^{1-4}$ The clinical presentation of AF is highly variable, ranging from the complete absence of symptoms to palpitations, heart failure, and hemodynamic collapse. Importantly, AF increases the risk of stroke about 5 times. ${ }^{5,6}$ AF doubles mortality and causes marked morbidity. ${ }^{7}$ Symptoms and complications of AF result from the irregular and often rapid ventricular response, loss of atrial contraction as well as from AFassociated hypercoagulability.

Studies, which compared rhythm control (cardioversion and subsequent prophylactic therapy against AF-recurrence) with rate control strategies showed that none of these two treatment strategies is superior. Rhythm control is more costly and inconvenient than rate control. ${ }^{8}$ However, it is very likely that safer and more effective anti-arrhythmic therapy would cause a clear trend towards an advantage of rhythm control.

Although strokes account for most of the functional impairment associated with AF, the arrhythmia can also decrease quality of life directly. In the Canadian Trial of Atrial Fibrillation (CTAF) study, quality of life improved after pharmacological antiarrhythmic treatment. $^{9}$ The post-cardioversion EMERALD (European and Australian Multicenter Evaluation Research on Atrial Dofetilide) study showed that dofetilide improved quality of life 1 month after electrical cardioversion. ${ }^{10}$ The randomized MANTRA-PAF (Medical ANtiarrhythmic Treatment or Radiofrequency Ablation in Paroxysmal Atrial Fibrillation) trial compared pulmonary vein isolation with 
radiofrequency ablation to antiarrhythmic drug therapy as a first-line rhythm control intervention in 294 patients. At 24-month follow-up, significantly more patients in the ablation group were free from symptomatic AF. Quality of life was significantly better in the ablation group at 12 and 24 months. However, total AF burden was not significantly different between both groups. ${ }^{11}$ Similar information has emerged from the results of the RAAFT II (Radiofrequency Ablation for Atrial Fibrillation Trial). ${ }^{12}$ Therefore, achieving sinus rhythm in $\mathrm{AF}$ is a central goal of treatment in $\mathrm{AF}$ patients. In order to develop new treatment strategies for AF, it is important to understand the underlying pathophysiological mechanisms of AF. Different forms of AF might require different types of treatment.

\section{Mechanisms of AF}

Theories of the mechanism of AF involve two main processes: ectopic focal discharges in one or several rapidly depolarizing foci and reentry involving one or more circuits (Figure 1). Both, reentry circuits as well as ectopic focal discharges can either be localized or they can intermittently change their location.

\section{Multiple Wavelets}

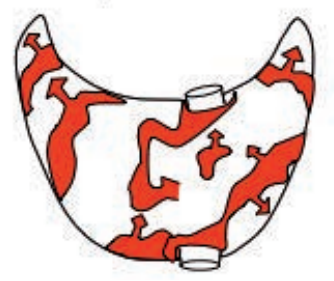

Multiple Foci

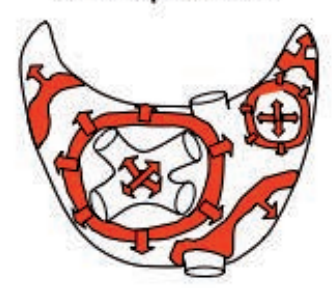

Focus $+$ Multiple Wavelets

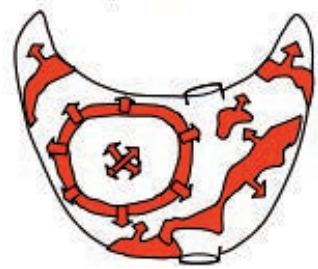

Figure 1: Different types of AF (modified from Eckstein et al.) 


\section{Focal atrial fibrillation}

Rapid activation originating from the pulmonary veins initiates AF in many patients with paroxysmal AF. ${ }^{13,14}$ Although some experimental studies have attempted to identify arrhythmogenic mechanisms in the pulmonary veins, ${ }^{15-18}$ the prominent role of the pulmonary veins in paroxysmal AF remains poorly understood. Histological studies have demonstrated cardiac muscle with preserved electrical properties extending into the pulmonary veins. ${ }^{19-22}$ Ectopic focal discharges as a trigger and perpetuator of $\mathrm{AF}$ are supported by experimental models of aconitine-induced $\mathrm{AF}^{23,24}$ in which the arrhythmia only persisted, when the ectopic focal discharges were present. The focal origin appears to be more important in patients with paroxysmal $\mathrm{AF}$ than in those with persistent $\mathrm{AF}$, and ablation of such foci may be curative. A better understanding of the mechanisms initiating 'focal' AF may help to develop safer and better ablation strategies and other therapeutic pharmacological modalities. Independent of the mechanisms involved, spontaneous atrial premature beats represent a potent trigger for $\mathrm{AF}$ initiating $\mathrm{AF}$-episodes in most cases. ${ }^{25,26}$

\section{Multiple-wavelet hypothesis}

The multiple-wavelet hypothesis as the mechanism of reentrant AF was proposed by Moe and colleagues, ${ }^{27}$ who suggested that continuous wave front and wavebreak interactions results in wavebreak thereby providing a steady source of fibrillation waves. The number of wavelets present at any time depends on the refractory period, mass, and conduction velocity in different parts of the atria. A large atrial mass with a short refractory period and delayed conduction may favor the occurrence of a large number of wavelets, stabilizing AF. 


\section{Atrial electrophysiological, structural and autonomic remodeling}

AF itself and several other conditions lead to an electrophysiological, structural and autonomic remodeling processes creating an arrhythmogenic substrate for AF. The complex interaction between electrophysiological, structural and autonomic remodeling processes in AF is summarized in figure 2. Sympathetic activation increases calcium-entry and the spontaneous release of calcium from the sarcoplasmic reticulum leading to atrial ectopies (trigger loop). Increased vagal activation together with $\mathrm{AF}$ induced atrial electrical remodeling shortens action potential duration facilitating reentry and thereby promoting AF. In the structural loop, atrial stretch during different conditions including congestive heart failure, hypertension or obstructive sleep apnea activates numerous profibrotic pathways resulting in atrial structural alterations and conduction disturbances, also facilitating reentrant mechanisms. The circular positive-feedback enhancement of these pathophysiological changes explains the general tendency of AF to become more stable with time. 


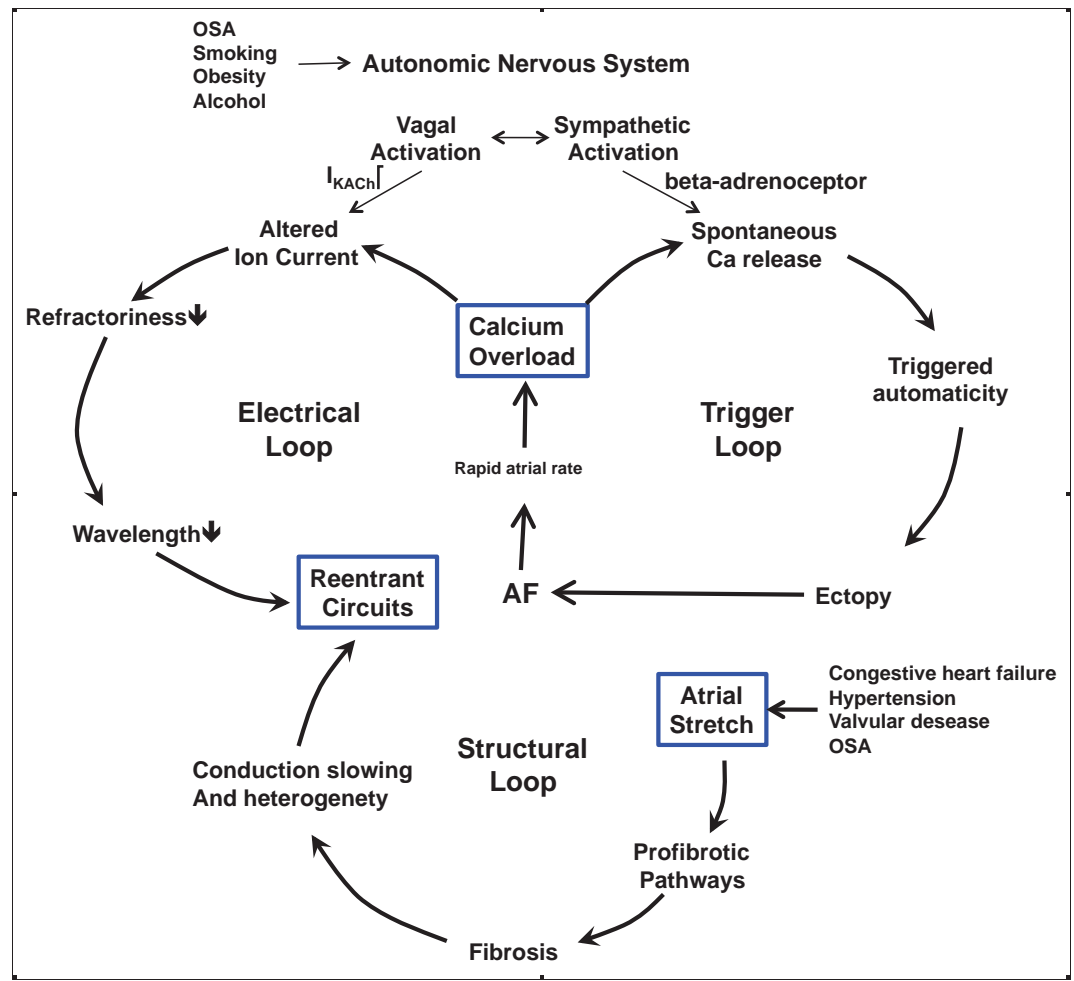

Figure 2: Interaction between electrophysiological, structural and autonomic remodeling (modified from Schotten et al. $^{28}$ ).

\section{Electrical remodeling and atrial fibrillation}

AF itself causes shortening in atrial refractoriness, thereby perpetuating AF and contributing to early AF recurrence after cardioversion, progressive drug resistance of longer-lasting AF, and progression from paroxysmal to more persistent forms of AF (AF begets $A F) .{ }^{29,30}$ Calcium enters atrial cells with each action potential. During rapid atrial rates, increased calcium-loading initiates autoprotective mechanisms that reduce calcium entry by calcium current inactivation and L-type calcium current downregulation. That decreases calcium loading by reducing action potential duration. Decreased action potential duration reduces wavelength thereby stabilizing reentrant circuits perpetuating $A F^{28,31-34}$ 


\section{Structural remodeling and atrial fibrillation}

Structural remodeling is characterized by increased atrial fibrosis formation and myocyte hypertrophy. Atrial tissue fibrosis impairs electrophysiological cell-to-cell coupling and conduction disturbances. ${ }^{28,31-36}$ Longterm AF leads to myocyte hypertrophy and increased endomysial fibrosis associated with electrical dissociation within the epicardial layer as well as between the epicardial layer and the endocardial bundle network. ${ }^{35}$ Besides AF alone, also other conditions cause structural alterations in the atrium. Longterm hypertension leads to atrial inflammation and fibrosis resulting in conduction abnormalities and increased inducibility of $\mathrm{AF}^{37}$ Congestive heart failure is associated with the development of a structural remodeling characterized by increased fibrosis formation and alterations in gap junction expression promoting heterogeneity of conduction and reentry. ${ }^{38}$ Longterm obstructive sleep apnea has been shown to be associated with significant atrial remodeling characterized by atrial enlargement and site-specific and widespread conduction abnormalities, and longer sinus node recovery in humans. ${ }^{39}$

\section{Atrial autonomic nervous system and AF}

Beta-adrenoceptor activation increases calcium-entry and the spontaneous release of calcium from the sarcoplasmic reticulum. This promotes delayed afterdepolarisation-related ectopic firing representing a potent trigger for $\mathrm{AF}^{28,40-43}$ Vagal discharges enhance acetylcholine dependent potassium currents (I KACh $_{\text {) }}$ reducing action potential duration thereby stabilizing reentry circuits perpetuating AF. In contrast, activation of sympathetic activity showed variable effects on action potential duration and atrial effective refractory period. ${ }^{28}$

Very early studies suggested that exercise-induced AF may be sympathetically driven; in contrast, the parasympathetic nervous system may be contributing to $A F$ in 
young patients with no structural heart disease. ${ }^{44}$ Several observations suggest that the autonomic nervous system plays an important role in both the initiation and the maintenance of AF. Studies in lone AF patients and in animal models of both intermittent rapid atrial pacing and congestive heart failure have indicated that $\mathrm{AF}$ onset is associated with simultaneous sympathovagal activation rather than with an increase in vagal or sympathetic drive alone. ${ }^{45-48}$ Ogawa et al, using direct nerve recordings from the stellate ganglia and vagal nerves, have shown increased sympathetic and vagal nerve discharges before the onset of atrial arrhythmias in dogs with pacing-induced congestive heart failure. ${ }^{47}$ Indeed, the atrial tachyarrhythmias in this model were prevented by prophylactic ablation of the stellate ganglion and the T2 to T4 thoracic sympathetic ganglia. ${ }^{47}$ The combination of vagal and sympathetic activity may provoke triggered activity (mainly late phase 3 EADs) acting as a potent initiator and perpetuator of AF. Combined sympatho-vagal activation can create a state with short action potential duration (parasympathetic stimulation) and an increase in calcium-load of the cells (sympathetic stimulation). This may explain observations that simultaneous sympathovagal discharge is associated with the onset of AF paroxysms in both patients and animal models (described above).

Additionally, in persistent AF patients and tachycardia-induced atrial remodeling in dogs atrial sympathetic hyperinnervation occurs, ${ }^{42-45}$ possibly promoting AF persistence and recurrence. In a dog model of pacing-induced congestive heart failure, $\mathrm{Ng}$ et al. recently demonstrated increased sympathetic and parasympathetic nerve growth in the left atrium; nerve growth was most pronounced in the pulmonary veins and the posterior wall of the left atrium. ${ }^{41}$ Jayachandran et al. demonstrated a heterogeneous increase in sympathetic innervation in the atria of dogs subjected to rapid atrial pacing for prolonged periods of time. ${ }^{42}$ There is also evidence of 
sympathetic hyperinnervation in patients with persistent $A F .{ }^{43}$ Interestingly, the cardiac autonomic system plays also a crucial role for the acute atrial electrical remodeling during atrial tachypacing since shortening in atrial effective refractory period could be inhibited by ganglionated plexi ablation. ${ }^{45}$

\section{Treatment strategies for AF}

As mechanisms of AF differ dependent on the underlying substrate, individualized therapy of AF might be a promising way to treat AF. Different strategies to treat AF are available today:

\section{Antiarrhythmic drugs}

Antiarrhythmic drugs have been traditionally defined as membrane-active agents, which modulate the opening and closing of ion channels, change the function of membrane pumps, and activate or block membrane receptors. ${ }^{49}$ This essentially results in antiarrhythmic electrophysiological changes characterized by an increase in refractoriness (by potassium channel blockers (class III antiarrhythmic drugs), e.g. sotalol, dofetilide, amiodarone) of the myocardium or a decrease in conduction velocity through the myocardium (by sodium channel blockers (class I antiarrhythmic drugs), e.g. flecainide). Additionally, some drugs, mainly sodium channel blockers, may decrease the firing rate of ectopic focal discharges. However, under certain conditions, anti-arrhythmic drugs do not only fail to display anti-arrhythmic effects but can also be proarrhythmic. ${ }^{49}$ Novel anti-arrhythmic drugs with conventional antiarrhythmic mechanisms are under investigation in AF, including newer multiplechannel blockers with a better safety profile and specific agents targeting atrial selective repolarization to avoid ventricular proarrhythmic effects. ${ }^{49}$ 


\section{Upstream therapy}

'Upstream' therapies to attenuate the development of a structural or electrical arrhythmogenic substrate is another approach to treat AF. Angiotensin-converting enzyme (ACE) inhibitors, angiotensin receptor blockers (ARBs), statins, and omega3 polyunsaturated fatty acids (PUFAs) have theoretical advantages as potential therapeutic strategies. ${ }^{28,30,34,50-53}$ In patients with structural heart disease ACEinhibitors and angiotensin inhibitors could reduce new onset AF. ${ }^{52-56}$ However, clinical studies failed to show sufficient efficacy of these approaches in patients without structural alterations. One year of angiotensin receptor blocker therapy per se does not reduce the number of $\mathrm{AF}$ episodes in patients with documented paroxysmal AF without structural heart disease..$^{57}$

Identification of novel pathways involved in the atrial remodeling process might provide new strategies to influence the development of an arrhythmogenic atrial structural substrate.

\section{Pulmonary vein ablation}

Catheter-based pulmonary vein ablation or isolation, surgically based maze operations, and atrioventricular node ablation for rate control may be effective nonpharmacological treatment strategies especially in younger individuals with paroxysmal AF and near-normal hearts. The European Society of Cardiology guidelines on management of AF released in 2010 have upgraded pulmonary vein ablation to first line therapy in patients with paroxysmal $A F^{58}$ The surgical maze procedure is presently limited to patients undergoing other heart surgery, e.g. mitral valve repair or replacement. However, as these operations become minimally invasive and highly effective, they are likely to be more widely used. ${ }^{59,60}$ 


\section{Modulation of the autonomic system}

Modulation of the autonomic nervous system might be a promising strategy to protect the myocardium from proarrhythmic autonomic influences and the development of electrical, autonomic and structural atrial remodeling. The most promising new strategy to modulate autonomic nervous system is renal sympathetic denervation.

Activation of renal efferent sympathetic nerve fibers increases sodium and water retention, reduce renal blood flow and elevate renin release from juxtaglomerular apparatus, regulating blood pressure ${ }^{61}$ Renal nerves contain sensory afferent fibers, which enable communication with the central nervous system. Activation of renal afferent nerves itself elevate sympathetic nervous outflow to the kidney and other downstream organs (see Figure 4).

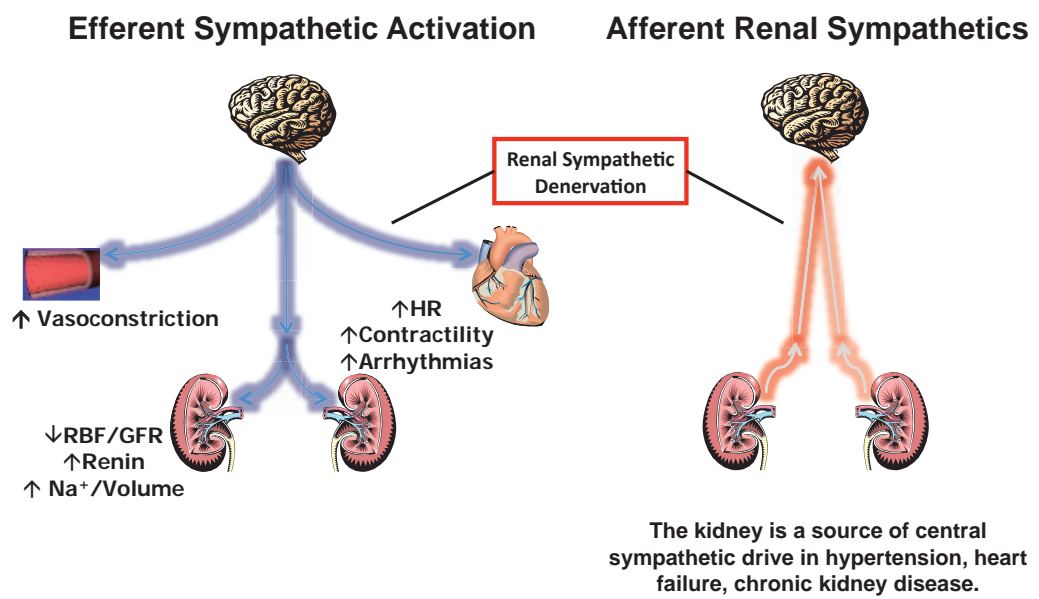

Figure 3: (modified by Schlaich et al. ${ }^{64}$ ). 
Recently, a catheter-based approach has been developed for renal sympathetic denervation (RDN). Special ablation catheters are inserted percutaneously via the femoral artery and advanced into the renal arteries. After placement, the catheter is withdrawn from distal to proximal segments and 4-8 ablations are administered within each artery. Both, a proof-of-concept trial (The Symplicity HTN-1 Trial ${ }^{62}$ ), including 45 patients with therapy-resistant hypertension (blood pressure $\geq 160 / 90$ $\mathrm{mmHg}$ despite receiving $\geq 3$ anti-hypertensive drugs) and a multicentre, prospective, randomized trial (The Symplicity HTN-2 Trial ${ }^{63}$ ) including 106 patients demonstrated that RDN significantly lowered blood pressure. Mechanistically, it has been observed that the procedure resulted in a $47 \%$ reduction of renal norepinephrine spillover measured with a radiochemical tracer methodology using $3 \mathrm{H}$-norepinephrine. ${ }^{62,64}$ Interestingly, firing of single sympathetic vasoconstrictor fibers, measured by single muscle sympathetic nerve activity were also reduced. ${ }^{65}$ These findings indicate a combined modulation of efferent and afferent signaling at the kidney by RDN. RDN may show antiarrhythmic effects by several mechanisms. Modulating of the central autonomic drive and of neuronal remodeling associated with AF (as described above) by RDN may reduce atrial arrhythmogenisis. Additionally, blood pressure reduction by RDN may attenuate the development of an atrial structural substrate. 


\section{Aim and outline of the study}

A better understanding of AF mechanisms is crucial to identify promising targets for pharmacological and device based therapy to prevent AF-initiation and perpetuation. Up to now, the arrythmogenic substrates due to different conditions like paroxysmal or permanent AF, congestive heart failure, hypertension, atrial dilation and aging, have already been studied in several animal models. ${ }^{31}$ However, the underlying mechanisms of arrhythmogenic triggers or electrical and structural alteration (substrate) associated with recently identified risk factors for AF like diabetes or obstructive sleep apnea are less understood. Additionally, although focal discharges are discussed to occur during $\mathrm{AF}$ and might represent an important factor for the perpetuation of $\mathrm{AF}$, the interaction of ectopic focal discharges with re-entry mechanisms during $\mathrm{AF}$ is unknown. A better understanding of these conditions might be important to optimize new treatment strategies of patients with AF.

The aim of this study was to develop and characterize animal models for AF maintained by (1) diabetes, (2) obstructive sleep apnea and (3) ectopic discharges to identify and test potential new antiarrhythmic treatment approaches:

1) Diabetes has been identified as a risk factor for AF. In a rat model for type 2 diabetes, we identified the underlying mechanisms involved in the development of the atrial arrhythmogenic substrate. We characterized the structural and electrophysiological alterations by electrophysiological measurements, magnetic resonance imaging, biochemical and histological measurements. Subsequently, the effect of pharmacological inhibition of cathepsin A, an alternative angiotensin splicing enzyme, which is highly upregulated in atria in diabetes, was be investigated (Chapter 2). 
2) Obstructive sleep apnea is associated with recurrence of AF after electrical cardioversion, pharmacological antiarrhythmic treatment and ablation based therapy. In pigs, the effect of obstructive respiratory events on atrial electrophysiology and AF-inducibility was investigated (Chapter 3). In this model, the effects of available AADs (Chapter 4) and modification of the autonomic system by renal denervation (Chapter 5) was tested.

3) The role of ectopic focal discharges for the inducibility and maintenance of AF is unknown. In goats, ectopic focal discharges were induced by epicardial application of aconitine and the interaction between AF and ectopic focal discharges were characterized. Additionally, the effect of the sodium blocker flecainide in this model was studied (Chapter 6).

\section{References}

1. Stewart S, Hart CL, Hole DJ, McMurray JJ. Population prevalence, incidence, and predictors of atrial fibrillation in the Renfrew/Paisley study. Heart. $2001 ; 86: 516-521$.

2. Stewart S, Hart CL, Hole DJ, McMurray JJ. A population-based study of the long-term risks associated with atrial fibrillation: 20-year follow-up of the Renfrew/Paisley study. Am J Med. 2002;113:359-364.

3. Fuster V, Ryden LE, Cannom DS, Crijns HJ, Curtis AB, Ellenbogen KA, Halperin JL, Le Heuzey JY, Kay GN, Lowe JE, Olsson SB, Prystowsky EN, Tamargo JL, Wann S, CN - TFoPG. ACC/AHA/ESC 2006 guidelines for the 
management of patients with atrial fibrillation-executive summary: a report of the American College of Cardiology/American Heart Association Task Force on Practice Guidelines and the European Society of Cardiology. Eur Heart J. 2006;27:1979-2030.

4. Miyasaka Y, Barnes ME, Gersh BJ, Cha SS, Bailey KR, Abhayaratna WP, Seward JB, Tsang TS. Secular trends in incidence of atrial fibrillation in Olmsted County, Minnesota, 1980 to 2000, and implications on the projections for future prevalence. Circulation. 2006;114:119-125.

5. Wolf PA, Abbott RD, Kannel WB. Atrial fibrillation as an independent risk factor for stroke: the Framingham Study. Stroke. 1991;22:983-988.

6. Hart RG, Halperin JL. Atrial fibrillation and stroke: concepts and controversies. Stroke. 2001;32:803-808.

7. Bornstein NM, Aronovich BD, Karepov VG, Gur AY, Treves TA, Oved M, Korczyn AD. The Tel Aviv Stroke Registry. 3600 consecutive patients. Stroke. 1996;27:1770-1773.

8. Camm AJ, Camm CF, Savelieva I. Medical treatment of atrial fibrillation. J Cardiovasc Med (Hagerstown). 2012;13:97-107.

9. Dorian P, Paquette M, Newman D, Green M, Connolly SJ, Talajic M, Roy D. Quality of life improves with treatment in the Canadian Trial of Atrial Fibrillation. Am Heart J. 2002; 143:984-990.

10. McClellan KJ, Markham A. Dofetilide: a review of its use in atrial fibrillation and atrial flutter. Drugs. 1999; 58:1043-1059. 
11. Cosedis Nielsen J, Johannessen A, Raatikainen P, Hindricks G, Walfridsson $\mathrm{H}$, Kongstad $\mathrm{O}$ et al. A randomized comparison of radiofrequency ablation and antiarrhythmia drug therapy as first line treatment in paroxysmal atrial fibrillation. N Engl J Med 2012:in press.

12. Wazni OM, Marrouche NF, Martin DO, Verma A, Bhargava M, SalibaW, Bash D, Schweikert R, Brachmann J, Gunther J, Gutleben K, Pisano E, Potenza D, Fanelli R, Raviele A, Themistoclakis S, Rossillo A, Bonso A, Natale A. Radiofrequency ablation vs antiarrhythmic drugs as firstline treatment of symptomatic atrial fibrillation: A randomized trial. JAMA 2005;293:2634-2640.

13. Calkins H, Brugada J, Packer DL, Cappato R, Chen SA, Crijns HJ, Damiano RJ, Jr., Davies DW, Haines DE, Haissaguerre M, lesaka Y, Jackman W, Jais P, Kottkamp H, Kuck KH, Lindsay BD, Marchlinski FE, M. HRS/EHRA/ECAS expert consensus statement on catheter and surgical ablation of atrial fibrillation: recommendations for personnel, policy, procedures and follow-up. A report of the Heart Rhythm Society (HRS) Task Force on Catheter and Surgical Ablatio. Europace. 2007;9:335-379.

14. Haissaguerre M, Jais P, Shah DC, Takahashi A, Hocini M, Quiniou G, Garrigue S, Le Mouroux A, Le Metayer P, Clementy J. Spontaneous initiation of atrial fibrillation by ectopic beats originating in the pulmonary veins. $\mathrm{N}$ Engl J Med. 1998;339:659-666.

15. Narayan SM, Kazi D, Krummen DE, Rappel WJ. Repolarization and activation restitution near human pulmonary veins and atrial fibrillation initiation: a mechanism for the initiation of atrial fibrillation by premature beats. J Am Coll Cardiol. 2008;52:1222-1230. 
16. Patterson E, Jackman WM, Beckman KJ, Lazzara R, Lockwood D, Scherlag BJ, Wu R, Po S. Spontaneous pulmonary vein firing in man: relationship to tachycardia-pause early afterdepolarizations and triggered arrhythmia in canine pulmonary veins in vitro. J Cardiovasc Electrophysiol. 2007;18:10671075.

17. Devereux RB, Lutas EM, Casale PN, Kligfield P, Eisenberg RR, Hammond IW, Miller DH, Reis G, Alderman MH, Laragh JH. Standardization of M-mode echocardiographic left ventricular anatomic measurements. J Am Coll Cardiol. $1984 ; 4: 1222-1230$.

18. Po SS, Li Y, Tang D, Liu H, Geng N, Jackman WM, Scherlag B, Lazzara R, Patterson E. Rapid and stable re-entry within the pulmonary vein as a mechanism initiating paroxysmal atrial fibrillation. J Am Coll Cardiol. 2005;45:1871-1877.

19. Spach MS, Barr RC, Jewett PH. Spread of excitation from the atrium into thoracic veins in human beings and dogs. Am J Cardiol 1972; 30: 844-854.

20. Nathan $\mathrm{H}$, Eliakim M. The junction between the left atrium and the pulmonary veins: an anatomic study of human hearts. Circulation 1966; 34: 412-422.

21. Zipes DP, Knope RF. Electrical properties of the thoracic veins. Am J Cardiol 1972; 29: 372-376.

22. Cheung DW. Electrical activity of the pulmonary vein and ist interaction with the right atrium in the guinea-pig. J Physiol (Lond) 1981; 314: 445-456.

23. Scherf D, Romano FJ, Terranova R. Experimental studies on auricular flutter and auricular fibrillation. Am Heart J 1948; 36: 241. 
24. Scherf D, Schaffer Al, Blumfeld S. Mechanism of flutter and fibrillation. Arch Intern Med 1953; 91: 241-251.

25. Jais $\mathrm{P}$, Haissaguerre $\mathrm{M}$, Shah $\mathrm{DC}$ et al. A focal source of atrial fibrillation treated by discrete radiofrequency ablation. Circulation 1997; 95: 572-576.

26. Chen SA, Hsieh MH, Tai CT et al. Initiation of atrial fibrillation by ectopic beats originating from the pulmonary veins: electrophysiological characteristics, pharmacological responses, and effects of radiofrequency ablation. Circulation 1999; 100: 1879-1886.

27. Moe GK, Abildskov JA. Atrial fibrillation as a self-sustaining arrhythmia independent of focal discharge. Am Heart J 58: 59-70, 1959.

28. Schotten U, Verheule S, Kirchhof P, Goette A. Pathophysiological mechanisms of atrial fibrillation: a translational appraisal. Physiol Rev. 2011; 91:265-325.

29. Wijffels MC, Kirchhof CJ, Dorland R, Allessie MA. Atrial fibrillation begets atrial fibrillation. A study in awake chronically instrumented goats. Circulation. 1995;92:1954-1968.

30. Yue L, Feng J, Gaspo R, Li GR, Wang Z, Nattel S. Ionic remodeling underlying action potential changes in a canine model of atrial fibrillation. Circ Res. 1997;81:512-525.

31. Nattel S. New ideas about atrial fibrillation 50 years on. Nature. 2002; 415:219 -226 . 
32. Wakili R, Voigt N, Kaab S, Dobrev D, Nattel S. Recent advances in the molecularpathophysiology of atrial fibrillation. J Clin Invest. 2011; $121: 2955-$ 2968.

33. Allessie MA, Boyden PA, Camm AJ, Kle'ber AG, Lab MJ, Legato MJ, Rosen MR, Schwartz PJ, Spooner PM, Van Wagoner DR, Waldo AL. Pathophysiology and prevention of atrial fibrillation. Circulation. 2001; 103:769 -777 .

34. Iwasaki YK, Nishida K, Kato T, Nattel S. Atrial fibrillation pathophysiology: implications for management. Circulation. 2011; 124:22642274.

35. Eckstein J, Maesen B, Linz D, Zeemering S, van Hunnik A, Verheule S, Allessie M, Schotten U. Time course and mechanisms of endo-epicardial electrical dissociation during atrial fibrillation in the goat. Cardiovasc Res. $2011 ; 89: 816-824$

36. Verheule S, Tuyls E, van Hunnik A, Kuiper M, Schotten U, Allessie M. Fibrillatory conduction in the atrial free walls of goats in persistent and permanent atrial fibrillation. Circ Arrhythm Electrophysiol. 2010; 3:590-599.

37. Lau DH, Mackenzie L, Kelly DJ, Psaltis PJ, Brooks AG, Worthington M, Rajendram A, Kelly DR, Zhang Y, Kuklik P, Nelson AJ, Wong CX, Worthley SG, Rao M, Faull RJ, Edwards J, Saint DA, Sanders P. Hypertension and atrial fibrillation: evidence of progressive atrial remodeling with electrostructural correlate in a conscious chronically instrumented ovine model. Heart Rhythm. 2010; 7:1282-1290. 
38. Li D, Fareh S, Leung TK, Nattel S. Promotion of atrial fibrillation by heart failure in dogs: atrial remodeling of a different sort. Circulation. 1999; 100:8795.

39. Dimitri H, Ng M, Brooks AG, Kuklik P, Stiles MK, Lau DH, Antic N, Thornton A, Saint DA, McEvoy D, Antic R, Kalman JM, Sanders P. Atrial remodeling in obstructive sleep apnea: implications for atrial fibrillation. Heart Rhythm. 2012; $9: 321-327$

40. Arora R. Recent insights into the role of the autonomic nervous system in the creation of substrate for atrial fibrillation: implications for therapies targeting the atrial autonomic nervous system. Circ Arrhythm Electrophysiol. 2012; 5:850-859.

41. Ng J, Villuendas R, Cokic I, Schliamser JE, Gordon D, Koduri H, Benefield B, Simon J, Murthy SN, Lomasney JW, Wasserstrom JA, Goldberger JJ, Aistrup GL, Arora R. Autonomic remodeling in the left atrium and pulmonary veins in heart failure: creation of a dynamic substrate for atrial fibrillation. Circ Arrhythm Electrophysiol. 2011; 4:388-396.

42. Jayachandran JV, Sih HJ, Winkle W, Zipes DP, Hutchins GD, Olgin JE. Atrial fibrillation produced by prolonged rapid atrial pacing is associated with heterogeneous changes in atrial sympathetic innervation. Circulation. 2000; $101: 1185-1191$.

43. Gould PA, Yii M, McLean C, Finch S, Marshall T, Lambert GW, Kaye DM. Evidence for increased atrial sympathetic innervation in persistent human atrial fibrillation. Pacing Clin Electrophysiol. 2006;29:821-829. 
44. Coumel P. Paroxysmal atrial fibrillation: a disorder of autonomic tone? Eur Heart J. 1994; 15(Suppl A):9-16.

45. Tan AY, Zhou S, Ogawa M, Song J, Chu M, Li H, Fishbein MC, Lin SF, Chen LS, Chen PS. Neural mechanisms of paroxysmal atrial fibrillation and paroxysmal atrial tachycardia in ambulatory canines. Circulation. 2008; 118:916-925.

46. Piccirillo G, Ogawa M, Song J, Chong VJ, Joung B, Han S, Magri D, Chen LS, Lin SF, Chen PS. Power spectral analysis of heart rate variability and autonomic nervous system activity measured directly in healthy dogs and dogs with tachycardia-induced heart failure. Heart Rhythm. 2009; 6:546-552.

47. Ogawa M, Zhou S, Tan AY, Song J, Gholmieh G, Fishbein MC, Luo H, Siegel RJ, Karagueuzian HS, Chen LS, Lin SF, Chen PS. Left stellate ganglion and vagal nerve activity and cardiac arrhythmias in ambulatory dogs with pacinginduced congestive heart failure. J Am Coll Cardiol. 2007; 50:335-343.

48. Lu Z, Scherlag BJ, Lin J, Niu G, Fung KM, Zhao L, Ghias M, Jackman WM, Lazzara $R$, Jiang $H$, Po SS. Atrial fibrillation begets atrial fibrillation: autonomic mechanism for atrial electrical remodeling induced by short-term rapid atrial pacing. Circ Arrhythm Electrophysiol. 2008; 1:184-192.

49. Ehrlich JR, Biliczki P, Hohnloser SH, Nattel S. Atrial-selective approaches for the treatment of atrial fibrillation. J Am Coll Cardiol. 2008;51:787-792.

50. Savelieva I, Camm J. Is there any hope for angiotensin-converting enzyme inhibitors in atrial fibrillation? Am Heart J. 2007;154:403-406. 
51. Savelieva I, Camm J. Statins and polyunsaturated fatty acids for treatment of atrial fibrillation. Nat Clin Pract Cardiovasc Med. 2008;5:30-41.

52. Vermes E, Tardif JC, Bourassa MG, Racine N, Levesque S, White M et al. Enalapril decreases the incidence of atrial fibrillation in patients with left ventricular dysfunction: insight from the Studies Of Left Ventricular Dysfunction (SOLVD) trials. Circulation 2003;107:2926-2931.

53. Maggioni AP, Latini R, Carson PE, Singh SN, Barlera S, Glazer R et al. Valsartan reduces the incidence of atrial fibrillation in patients with heart failure: results from the Valsartan Heart Failure Trial (Val-HeFT). Am Heart J 2005;149:548-557.

54. Ducharme A, Swedberg K, Pfeffer MA, Cohen-Solal A, Granger CB, Maggioni AP et al. Prevention of atrial fibrillation in patients with symptomatic chronic heart failure by candesartan in the Candesartan in Heart failure: Assessment of Reduction in Mortality and morbidity (CHARM) program. Am Heart $J$ 2006;152:86-92.

55. Massie BM, Carson PE, McMurray JJ, Komajda M, McKelvie R, Zile MR et al. Irbesartan in patients with heart failure and preserved ejection fraction. N Engl J Med 2008;359:2456-2467.

56. Healey JS, Baranchuk A, Crystal E, Morillo CA, Garfinkle M, Yusuf S et al. Prevention of atrial fibrillation with angiotensin-converting enzyme inhibitors and angiotensin receptor blockers: a meta-analysis. J Am Coll Cardiol 2005;45:1832-1839. 
57. Goette A, Schön N, Kirchhof P, Breithardt G, Fetsch T, Häusler KG, Klein HU, Steinbeck G, Wegscheider K, Meinertz T. Angiotensin Il-antagonist in paroxysmal atrial fibrillation (ANTIPAF) trial. Circ Arrhythm Electrophysiol. 2012;5:43-51.

58. Guidelines for the management of atrial fibrillation: the Task Force for the Management of Atrial Fibrillation of the European Society of Cardiology (ESC). European Heart Rhythm Association; European Association for Cardio-Thoracic Surgery, Camm AJ, Kirchhof P, Lip GY, Schotten U, Savelieva I, Ernst S, Van Gelder IC, Al-Attar N, Hindricks G, Prendergast B, Heidbuchel H, Alfieri O, Angelini A, Atar D, Colonna P, De Caterina R, De Sutter J, Goette A, Gorenek B, Heldal M, Hohloser SH, Kolh P, Le Heuzey JY, Ponikowski P, Rutten FH. Eur Heart J. 2010;31:2369-429.

59. Jahangiri M, Weir G, Mandal K, Savelieva I, Camm J. Current strategies in the management of atrial fibrillation. Ann Thorac Surg. 2006;82:357-364.

60. Maesen B, Nijs J, Maessen J, Allessie M, Schotten U. Post-operative atrial fibrillation: a maze of mechanisms. Europace. 2012;14:159-174.

61. DiBona GF, Kopp UC: Neural control of renal function. Physiol Rev. 1997; 77:75-197.

62. Krum H, Schlaich M, Whitbourn R, Sobotka PA, Sadowski J, Bartus K, Kapelak B, Walton A, Sievert H, Thambar S, Abraham WT, Esler M. Catheterbased renal sympathetic denervation for resistant hypertension: a multicentre safety and proof-of-principle cohort study. Lancet. 2009; 373:1275-1281. 
63. Symplicity HTN-2 Investigators, Esler MD, Krum H, Sobotka PA, Schlaich MP, Schmieder RE, Böhm M. Renal sympathetic denervation in patients with treatment-resistant hypertension (The Symplicity HTN-2 Trial): a randomised controlled trial. Lancet. 2010; 376:1903-1909.

64. Schlaich MP, Sobotka PA, Krum H, Lambert E, Esler MD. Renal sympatheticnerve ablation for uncontrolled hypertension. N Engl J Med. 2009; 361:932934.

65. Hering D, Lambert EA, Marusic P, Walton AS, Krum H, Lambert GW, Esler MD, Schlaich MP. Substantial reduction in single sympathetic nerve firing after renal denervation in patients with resistant hypertension. Hypertension. $2013 ; 61: 457-464$. 
Chapter 2

CathepsinA-Inhibition Reduces Susceptibility to Atrial Tachyarrhythmia and Protects Atrial Emptying Function in Old Zucker Diabetic Fatty Rats.

Dominik Linz, Stefan Dhein, Hans-Paul Juretschke, Claudia NeumannHaefelin, Sven Ruf, Paulus Wohlfart, Thorsten Sadowski, Hartmut Rütten, Michael Böhm, Ulrich Schotten.

Submitted 


\section{Abstract}

Introduction: Type 2 diabetes (T2D) is an independent risk factor for atrial fibrillation $(\mathrm{AF})$ and stroke and is associated with decreased exercise tolerance and cardiomyopathy. Cathepsin A (CathA) is a lysosomal enzyme involved in bradykinin and angiotensin metabolism. We tested the hypothesis that CathA-inhibition protects against atrial remodeling in old Zucker diabetic fatty-rats (ZDF), an animal model for T2D. Method: Three groups of rats were studied: ZDF receiving vehicle $(n=20), Z D F$ receiving CathA-inhibitor (SAR); $50 \mathrm{mg} / \mathrm{kg}$ by gavage; $n=20$ ), and their age matched lean littermates (LEAN, $n=20$ ). Left atrial (LA) emptying function (magnetic resonance imaging) and atrial electrophysiological parameters were measured before the hearts were harvested for histological and biochemical analysis. Results: At the age of 9.5 months, ZDF-rats showed increased LA-CathA-activity, an increase in AF susceptibility, and reduced atrial contractile function. CathA-inhibition decreased the median duration of LA-tachyarrhythmia $(12.0 \pm 1.7 \mathrm{~s}$ vs. $1.2 \pm 0.47 \mathrm{~s}$, $p<0.01)$ induced by burst pacing. Total atrial activation times and regions of slowest conduction (local conduction times longer than $2 \mathrm{~ms}, 4.3 \pm 1.7 \%$ vs. $0.3 \pm 0.5 \%, p<0.01$ ) were reduced. Cardiac magnetic resonance imaging revealed better LA-emptying function parameters (active percent emptying, $29 \pm 1 \%$ vs. $23 \pm 2 \%, p<0.01$ ) and normal LA-dimensions after CathA-inhibitor-treatment compared with untreated ZDF. Inhibition of CathA-activity resulted in reduced atrial bradykinin degradation and decreased LA-fibrosis formation, Cx43 lateralization and osteopontin gene expression in ZDF-rats. Conclusion: CathA-inhibition prevents the development of arrhythmogenic remodeling and contractile dysfunction in Zucker diabetic fatty-rats. These data suggest a causal relationship between atrial CathA-activity and atrial remodeling in $\mathrm{T} 2 \mathrm{D}$. 


\section{Introduction}

Atrial fibrillation (AF) is the most common type of sustained tachyarrhythmia and is associated with significant morbidity and mortality. ${ }^{1}$ Type 2 diabetes (T2D) is a strong independent risk factors for atrial fibrillation and stroke..$^{2,3,4}$ In a rat model, diabetes has been shown to produce an arrhythmogenic substrate in the atrium with an increase in AF susceptibility. ${ }^{5,6}$ Additionally, an abnormal response to exercise is frequently observed in asymptomatic diabetic patients without any clinical evidence for further cardiovascular disease..$^{7,8,9}$ The reduced exercise tolerance might be related to atrial and/or ventricular contractile dysfunction frequently seen in diabetic patients. $^{10}$

Cathepsin A (CathA) is a lysosomal serine protease, which is also found in the extracellular space. It has been suggested to play an important role in degradation of extracellular peptides and inflammation. ${ }^{11,12}$ CathA belongs to a group of enzymes mediating alternate routes for angiotensin formation and bradykinin breakdown. Evidence of upregulation of alternative angiotensin-generating enzymes like chymase or cathepsins has been described in many pathologies e.g. in myocardial infarction, congestive heart failure or diabetes. ${ }^{13,14,15}$ Pharmacological inhibition of CathA by the natural product ebelactone $\mathrm{B}^{16}$ and antisense oligonucleotides suppressing the expression of Cath $^{17}$ increased renal bradykinin levels and prevented deoxycorticosterone acetate-salt hypertension in rats. Treatment with $\mathrm{SAR}^{18}$, an orally available CathA inhibitor, has been shown to result in a reduction of cardiac hypertrophy in apolipoprotein E knockout mice with continuous angiotensin II infusion and prevented $A F$ in rats with ventricular ischemia/reperfusion, a rat model for atrial structural remodeling ${ }^{18}$. 
As activation of the renin-angiotensin system might also be involved in atrial remodeling during diabetes ${ }^{6}$, we tested the protective effect of the selective and potent orally available CathA inhibitor SAR ((S)-3-\{[1-(2-Fluoro-phenyl)-5-methoxy1H-pyrazole-3-carbonyl]-amino\}-3-(2-methylphenyl)-propionic acid, characterized as $2 \mathrm{a} i{ }^{18}$ ) on atrial emptying function and atrial electrical and structural arrhythmogenic remodeling in old Zucker Diabetic Fatty rats (ZDF), an animal model for T2D.

\section{Methods}

\section{Animal model}

Zucker diabetic fatty-rats (ZDF/Gmi-fa/fa, $n=40)$ and their age matched lean littermates (male, 200 - 250g body weight, 10 weeks old, LEAN, $\mathrm{n}=20$ ) were purchased from Charles River and housed three per cage under standardized conditions (room temperature $24^{\circ} \mathrm{C}$, relative humidity $60 \%$, lighting times 12 hours dark/light cycle). They had free access to a standardized diet, sodium content $0.2 \%$, Altromin, Lage, Germany) and tap drinking water. ZDF-rats were randomized in 2 groups: placebo treated ZDF-rats (ZDF-placebo, $n=20$ ) and CathA-inhibitor $(S A R)^{18}$ treated ZDF-rats (ZDF-SAR, $\mathrm{n}=20$ ). SAR was synthesized at Sanofi-Aventis, Frankfurt am Main, and administered daily via oral gavage $(50 \mathrm{mg} / \mathrm{kg} / \mathrm{d}$, Vehicle: 0,5\% HEC / Tween 5\%, volume administered: $1 \mathrm{ml} / \mathrm{kg}$ ). ZDF-placebo rats were treated with vehicle only. After 38 weeks, animals were anesthetized and cardiac function was assessed by cardiac magnet resonance imaging (MRI). One week later, electrophysiological measurements were performed in open chest experiments and the animals were sacrificed. Hearts were rapidly removed, trimmed free from non 
cardiac tissues, and weighed. Left atrial (LA) tissue was frozen in liquid nitrogen for biochemical analysis or stored in formalin for histological examination.

All animal studies conform with the guide for the Care and Use of laboratory Animals published by the US National Institutes of Health (NIH Publication No. 85-23, revised 1996).

\section{Electrophysiological studies}

All measurements were performed during general anesthesia by intraperitoneal injection of pentobarbital at the age of 9.5 months. Surface ECG (lead II) was recorded via subcutaneous needle electrodes. To analyze atrial conduction, a custom made mapping electrode with $4 * 5$ unipolar electrodes $(1.0 \mathrm{~mm}$ interelectrode distance) was placed on the LA-free wall. Unipolar signals were recorded using a custom made channel mapping amplifier (filtering bandwidth $0.1-408 \mathrm{~Hz}$, sampling rate $1 \mathrm{kHz}, \mathrm{A} / \mathrm{D}$ resolution 16 bits). Unipolar pacing was performed from the surface of the right atrium (pulse width of $1 \mathrm{~ms}$ at twice the diastolic threshold). Maps from 5 consecutive beats were analyzed. Local activation times were identified by maximum negative $\mathrm{dV} / \mathrm{dt}$ in each unipolar electrogram. Local activation time differences were calculated between neighboring electrodes (conduction times). Conduction times of $\geq 3 \mathrm{~ms}$ (equivalent to conduction velocities $\leq 33 \mathrm{~cm} / \mathrm{s}$ ) were considered as being prolonged. ${ }^{19}$ Total-atrial activation time was defined as the time difference between the right atrial activation time, determined from a MAP-catheter (Franz-like electrode) next to the pacing electrode, and the latest left atrial activation time recorded by the mapping electrode during right atrial-pacing (cycle length: 150ms). Atrial effective refractory period (AERP) was measured at a basic cycle length of $150 \mathrm{~ms}$ at the free

wall of the LA. The mean of 3 AERP measurements was used for analysis. 
Susceptibility to atrial tachyarrhythmia/atrial fibrillation (AT/AF) induced by repetitive $1 \mathrm{~s}$ bursts of stimuli (very rapid pacing, cycle length: $10 \mathrm{~ms}$ ). When atrial electrograms showed a rapid atrial rate, cycle length $<70 \mathrm{~ms}$ and duration $>5$ beats, AT/AF was diagnosed. The duration of the longest of three subsequent episode of AT/AF induced during this protocol was measured and was taken as AF/AT-duration.

\section{Magnetic resonance imaging (MRI)}

All scans were acquired with a 7-T magnetic resonance imaging (MRI) scanner (Bio Spec $70 / 30$ ) in all rats at the age of 9.5 months (anesthesia: $1.5-2.5 \%$ isoflurane). Multi-slice short-axis cine imaging was performed from the upper left atrial roof to the apex with a slice thickness of $1.2 \mathrm{~mm}$ and an inter-slice gab of $0 \mathrm{~mm}$. Orthogonal long axis cine imaging of the whole heart, two chamber and four chamber views, were acquired. Boundaries of the left ventricle and left atrium were obtained in each short-axis image. The LV-volumes were then determined by Simpson's rule. LA-area was manually encircled. The point of insertion of the mitral valve leaflets was taken as the atrioventricular border. Pulmonary veins were excluded at their ostia and the left atrial appendage was excluded at its base. All dimensions were measured throughout the cardiac cycle. ${ }^{20}$ The cardiac cycle was divided into 10 equal phases with an interphase time difference of the spontaneous cycle length / 10. The MRIimages of all LAs were analyzed by the same blinded investigator.

LV-enddiastolic volume (LVedV) and end-systolic volume (LVesV) were derived from the LA-volume/time curves for maximal and minimal values. LV-ejection fraction (EF) was subsequently computed. Minimal LA- (LA min) and maximal LA-volume (LAmax) and their difference (cyclical change volume) were determined from the LAvolume/time curves. The minimal volume at the end of rapid passive emptying (LAre) and the volume before active emptying (LApc) were determined from the volume- 
time curves as described elsewhere. ${ }^{21}$ LA-emptying function parameters (total percent emptying ((LAmax-LAmin)/LAmax), active percent emptying ((LApcLAmin)/LApc), passive percent emptying ((LAmax-LAre)/LAmax) were computed according to Jarvinen et al.. ${ }^{20}$

\section{Measurement of LV-pressure and blood pressure}

LV-enddiastolic pressure (LVedP) was measured in anaesthetized rats using Millar Tip catheters (Millar Instruments Inc, Houston, USA) introduced from the right arteria carotis and advanced into the left ventricle. In conscious rats, blood pressure was measured using the tailcuff method.

\section{Gene expression analysis}

Gene expression analysis was performed by realtime PCR. Total RNA was extracted from whole LA by homogenizing in RLT buffer and by using RNeasy Mini columns (Qiagen, Hidlen, Germany). Genomic DNA impurities were removed by DNase treatment (DNA removal kit; Ambion, Austin, TX), and cDNA was synthesized by reverse transcription (Life Science Technologies, Darmstadt, Germany). Quantitative real-time RT-PCR was performed using the Taqman primers on an ABI Prism 7500 Sequence Detector (Applied Biosystems, Foster City, CA) and c(t) values obtained by using a respective software (SDS version 1.9). Gene expression was normalized to glyceraldehyde-3-phosphate dehydrogenase (GAPDH) as housekeeping gene. For each analysis, mean values were used from 8-10 animals per group. Probes used to amplify the transcripts were as follows (purchased by Applied Biosystems): CathA (Rn01424035_g1); angiotensin converting enzyme (ACE, Rn00561094_m1); angiotensin receptor 1a, (AGTR1a, Rn01435427_m1); angiotensin receptor 1b (AGTR1b, Rn02132799_s1), Chymase (Rn00565319_m1); osteopontin (OPN, 
Rn00681031_m1); tissue growth factor B1 (TGFB1, Rn00572010_m1); collagen 1a1 (Col1a1, Rn01463848_m1); matrix metalloproteinase-2 (MMP2, Rn01538174_m1); tissue inhibitors of metalloproteinases1 (TIMP, Rn00561661_m1); atrial natriuretic peptide (ANP, Rn00561661_m1); glyceraldehyde 3-phosphate dehydrogenase (GAPDH, Rn99999916_s1).

\section{CathepsinA activity, angiotensin and bradykinin degradation}

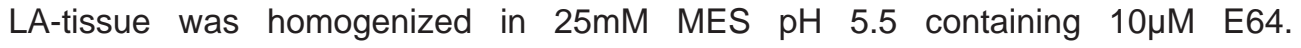
Homogenates were then mixed with SAR or DMSO in a multiple assay plate. After incubation for 15 minutes at room temperature a bradykinin peptide carrying a $\mathrm{N}$ terminal BodipyFL label (Jerini Peptide Technology) was added to the assay mixture to a final concentration of $2 \mu \mathrm{M}$. After incubation for $15 \mathrm{~min}$ at room temperature the reaction was stopped by the addition of $130 \mathrm{mM}$ Hepes $\mathrm{pH} 5.5$ and containing 0.013\% Triton X-100, 0.13\% Coating Reagent (Caliper Life Sciences), 6.5\% DMSO and $20 \mu \mathrm{M}$ ebelactone B (Sigma, \#E0886). Uncleaved substrate and product were then separated by a microfluidic capillary electrophoresis on a LabChip® 3000 Drug Discovery System (12-Sipper-Chip, Caliper Life Sciences) and quantified by determination of the respective peak areas. Substrate turnover was calculated by dividing product peak area by the sum of substrate and product peak areas. The difference in bradykinin turnover in the presence and absence of SAR at pH 5.5 (optimum of carboxypeptidase activity) was used to quantify CathA-activity. Additionally, bradykinin turnover at $\mathrm{pH} 7.4$ was quantified in all groups. Angiotensin was quantified in LA-preparations by ELISA. 


\section{Histology and connexins}

Separated LA fixed in $4 \%$ formaldehyde for $24 \mathrm{~h}$ and imbedded in paraffin were used for histological examination and gap junction analysis. Tissue sections of $5 \mu \mathrm{m}$ were fixed at $56^{\circ} \mathrm{C}$ overnight, deparaffinized, rehydrated and stained with hematoxylin and eosin (H\&E) to determine cardiomyocyte diameter and cell distances. To visualize tissue fibrosis amount, the sections were stained with Picro-Sirius Red. The percentage of the LA consisting of interstitial collagen was calculated as the ratio of Picro-Sirius-Red positively stained area over total LA-tissue area, excluding blood vessels and the epi- and endocardial plane, using ImageJ 1.37a (National Institute of Health, USA). Fibrosis was quantified on three sections per atrium (6-8 fields per section).

Immunostaining for connexin43 $(\mathrm{Cx} 43)$ in formalin fixed left atrial tissue from 9 LEAN, 9 ZDF-placebo and 8 ZDF-SAR rats was performed like described elsewhere. $^{22}$ Briefly, expression of $\mathrm{Cx} 43$ at the lateral and polar membrane of cardiomyocytes from rat atria was investigated. Per animal 2-3 sections were analyzed, and per section 5-10 cells, so that each data point of the columns is representing 100-250 cells.

\section{Blood samples}

The concentration of BNP32 (fluorescent ELISA) and osteopontin was determined in plasma using a radio immunoassay (Phoenix Pharmaceuticals Inc., USA). Serum ACE-activity was determined by a fluorimetric assay according to Santos et al. 


\section{Statistical Analysis}

The data are expressed as mean $\pm S E M$. Statistical significance was assessed by analysis of variance (ANOVA), followed by the unpaired Student's t tests (two-tailed) for 2 groups. If necessary, Dunnett's test was employed. P-values of $<0.05$ were considered statistically significant. 


\section{Results}

Increase in atrial Cathepsin A activity in ZDF

LA-CathA-activity was significantly increased in ZDF-placebo, and this increase was effectively inhibited by SAR treatment (Figure 1). This was associated with increased CathA-gene expression in ZDF-placebo compared to LEAN (1.49 \pm 0.14 -fold, $p<0.001)$.

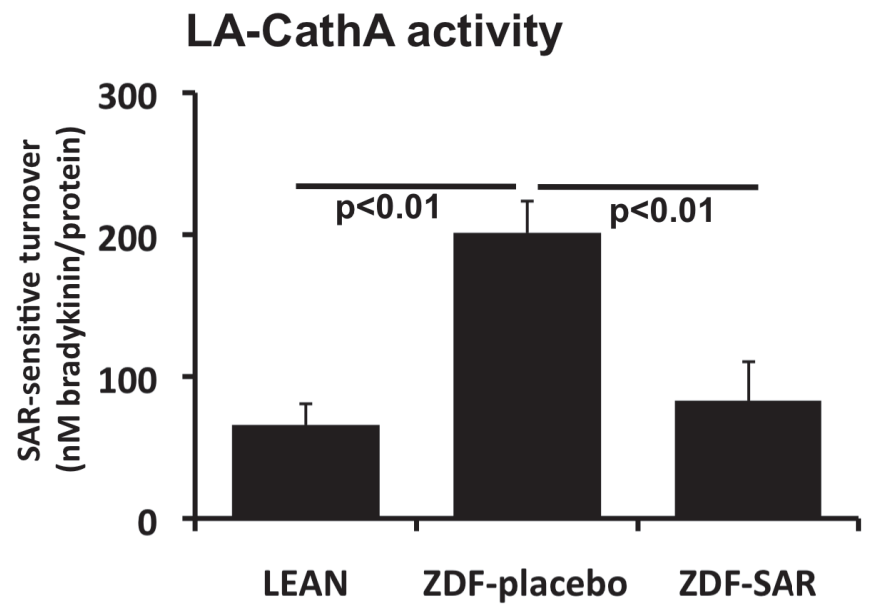

Figure 1: CathepsinA-activity (SAR-sensitive bradykinin turnover) in left atrial preparations in LEAN, in ZDF-placebo and in ZDF-SAR. 


\section{Metabolic and hemodynamics parameters}

Metabolic and hemodynamic parameters from these experiments are summarized in Table 1. At the age of 9.5 months, increased concentration of glucose and HbA1c in serum of ZDF-placebo was accompanied with severe signs of nephropathy and dyslipidemia. SAR-treatment did not change metabolic parameters or renal function in ZDF-rats at the age of 9.5 months. Cardiac hypertrophy index, expressed as heart weight / 100g body weight, was neither elevated in hearts from ZDF-placebo nor from SAR-treated ZDF-rats in comparison to LEAN. Body weights between untreated and SAR-treated ZDF-rats were not different. However, LEAN were heavier than ZDF-rats.

LV-EF and LV-dimensions determined by MRI did not differ between ZDF and LEAN, but LV-enddiastolic pressure was increased in ZDF, indicating the development of moderate diastolic dysfunction with preserved systolic function in old ZDF-rats. Blood pressure measured in conscious rats (tail-cuff-methode) revealed no differences between ZDF-placebo and LEAN, but heart rate tended to be lower in ZDF-rats. SAR-treatment in old ZDF-rats did not significantly change blood pressure nor heart rate in ZDF-rats. Lower LVedP and plasma BNP-levels $(121.3 \pm 1.5 \mathrm{ng} / \mathrm{ml}$ vs. $159.9 \pm 11.3 \mathrm{ng} / \mathrm{ml}$ in ZDF, $\mathrm{p}<0.001$ ) were preserved in SAR-treated ZDF-rats. LAdimensions determined by LA-volume/heart weight indices and metabolic parameters were not affected by SAR treatment. 
Table 1: General Characteristics

\begin{tabular}{|c|c|c|c|}
\hline & LEAN & $\begin{array}{l}\text { ZDF- } \\
\text { placebo }\end{array}$ & ZDF-SAR \\
\hline \multicolumn{4}{|l|}{ Characteristics } \\
\hline Bodyweight (g) & $472.7 \pm 7.8$ ** & $337.5 \pm 6.3$ & $328.1 \pm 6.7$ \\
\hline Heart weight (g) & $1.25 \pm 0.01 *$ & $1.06 \pm 0.03$ & $0.99 \pm 0.01$ \\
\hline LV-weight (g) & $0.91 \pm 0.02 *$ & $0.77 \pm 0.09$ & $0.71 \pm 0.04$ \\
\hline LA-weight (g) & $\underset{*}{0.022 \pm 0.001}$ & $0.015 \pm 0.001$ & $0.015 \pm 0.002$ \\
\hline Heart weight/100g body weight & $0.27 \pm 0.02$ & $0.32 \pm 0.02$ & $0.31 \pm 0.01$ \\
\hline LV-weight/100g body weight & $0.21 \pm 0.01$ & $0.23 \pm 0.01$ & $0.22 \pm 0.01$ \\
\hline LA-weight/100g body weight & $\begin{array}{c}0.0046 \pm 0.00 \\
02\end{array}$ & $\begin{array}{c}0.0043 \pm 0.00 \\
03\end{array}$ & $\begin{array}{c}0.0045 \pm 0.00 \\
07\end{array}$ \\
\hline \multicolumn{4}{|l|}{ Diabetes and metabolism } \\
\hline Glucose (mmol/l plasma) & $6.26 \pm 0.16$ ** & $28.68 \pm 0.97$ & $28.21 \pm 0.97$ \\
\hline HbAlc (\%) & $4.15 \pm 0.03 * *$ & $10.1 \pm 0.07$ & $9.49 \pm 0.01$ \\
\hline Albumin/creatinin-ratio & $0.07 \pm 0.01 * *$ & $12.96 \pm 1.75$ & $9.37 \pm 1.01$ \\
\hline Creatinine-clearance & $7.4 \pm 0.2 * *$ & $9.3 \pm 0.3$ & $9.3 \pm 0.2$ \\
\hline LDL (mmol/l) & $0.30 \pm 0.01 * *$ & $0.63 \pm 0.06$ & $0.66 \pm 0.06$ \\
\hline Triglyceride $(\mathrm{mmol} / \mathrm{l})$ & $1.37 \pm 0.06$ ** & $5.02 \pm 0.34$ & $4.28 \pm 0.19$ \\
\hline \multicolumn{4}{|l|}{ Blood pressure (tailcuff) } \\
\hline Blood pressure $(\mathrm{mmHg})$ & $141.3 \pm 2.2$ & $141.5 \pm 3.1$ & $141.5 \pm 3.9$ \\
\hline Heart rate (bpm) & $339.2 \pm 8.0$ & $327.1 \pm 6.8$ & $329.8 \pm 7.7$ \\
\hline \multicolumn{4}{|l|}{ Ventricular haemodynamics } \\
\hline \multicolumn{3}{|l|}{ LV-function (MRI) } & $5.2 \pm 0.5 *$ \\
\hline $\mathrm{EF}(\%)$ & $66.6 \pm 0.93$ & $64.6 \pm 0.65$ & $63.6 \pm 0.58$ \\
\hline $\operatorname{EDV}(\mu l)$ & $711 \pm 15.6 *$ & $646 \pm 21.9$ & $605 \pm 8$ \\
\hline $\begin{array}{l}\text { ED-Vol. Index }(\mu \mathrm{l} / \mathrm{g} \\
\text { BW) }\end{array}$ & $1.52 \pm 0.03 * *$ & $1.87 \pm 0.07$ & $1.83 \pm 0.04$ \\
\hline $\mathrm{ESV}(\mu \mathrm{l})$ & $239 \pm 9.5$ & $229 \pm 9.8$ & $220 \pm 4.3$ \\
\hline $\begin{array}{l}\text { ES-Vol. Index }(\mu \mathrm{l} / \mathrm{g} \\
\text { BW) }\end{array}$ & $0.51 \pm 0.02 *$ & $0.66 \pm 0.03$ & $0.66 \pm 0.02$ \\
\hline \multicolumn{4}{|l|}{ LA-dimension (MRI) } \\
\hline $\operatorname{LAD}(\mathrm{cm})$ & $0.52 \pm 0.02 *$ & $0.48 \pm 0.01$ & $0.47 \pm 0.02$ \\
\hline LAV (ml) & $0.43 \pm 0.01 * *$ & $0.33 \pm 0.01$ & $0.32 \pm 0.01$ \\
\hline LAV-index ( $\mu$ l/g BW) & $0.91 \pm 0.02$ & $0.94 \pm 0.03$ & $0.97 \pm 0.02$ \\
\hline LAV-index $(\mu \mathrm{l} / \mathrm{g} \mathrm{HW})$ & $0.29 \pm 0.02$ & $0.31 \pm 0.03$ & $0.31 \pm 0.02$ \\
\hline
\end{tabular}

$p<0.01$, vs. ZDF-placebo **

$p<0.05$, vs. ZDF-placebo * 


\section{Atrial electrophysiology}

Short episodes of AF/AT could be induced in all rats investigated in our study. The median duration of AF/AT was increased in ZDF-placebo in comparison to LEAN (Figure 2 A). Total atrial activation time was significantly longer (Figure 2 B) and the percentage of regions with slow conduction during rapid pacing was higher (Figure 2 C) in ZDF-placebo compared to LEAN. AERP was prolonged in ZDF-placebo when compared to LEAN (Figure 2 D). Importantly, SAR-treatment reduced median AF/AT-duration in ZDF-rats to values comparable to the LEAN animals. SARtreatment did not attenuate AERP-prolongation in ZDF-rats but reduced total atrial activation time and the percentage of regions with slow conduction.

\section{LA-emptying function}

LA-emptying function parameters are shown in figure 3. LA-fractional shortening was reduced in ZDF-placebo when compared to LEAN $(15.4 \pm 1.5 \%$ vs. $17.3 \pm 0.9 \%$, $p<0.001)$. Global LA-emptying function as assessed by total percent emptying was not changed (Figure 3 B). In contrast, active percent emptying was diminished in old ZDF-placebo compared to LEAN (Figure $3 \mathrm{E}$ ). SAR-treatment attenuated changes in LA-fractional shortening and active percent emptying. During the MRI-scan, heart rate was lower in ZDF-placebo and ZDF-SAR compared to LEAN (261 \pm 3.34 bpm, $260 \pm 5.67 \mathrm{bpm}$ and $281 \pm 5.37 \mathrm{bpm}$ respectively) $(p<0.05)$. However, since atrial emptying function is inversely correlated to heart rate, this would even lead to underestimation of the differences between LEAN and ZDF-placebo and ZDF-SAR and would not influence the comparison between ZDF-placebo and ZDF-SAR. 

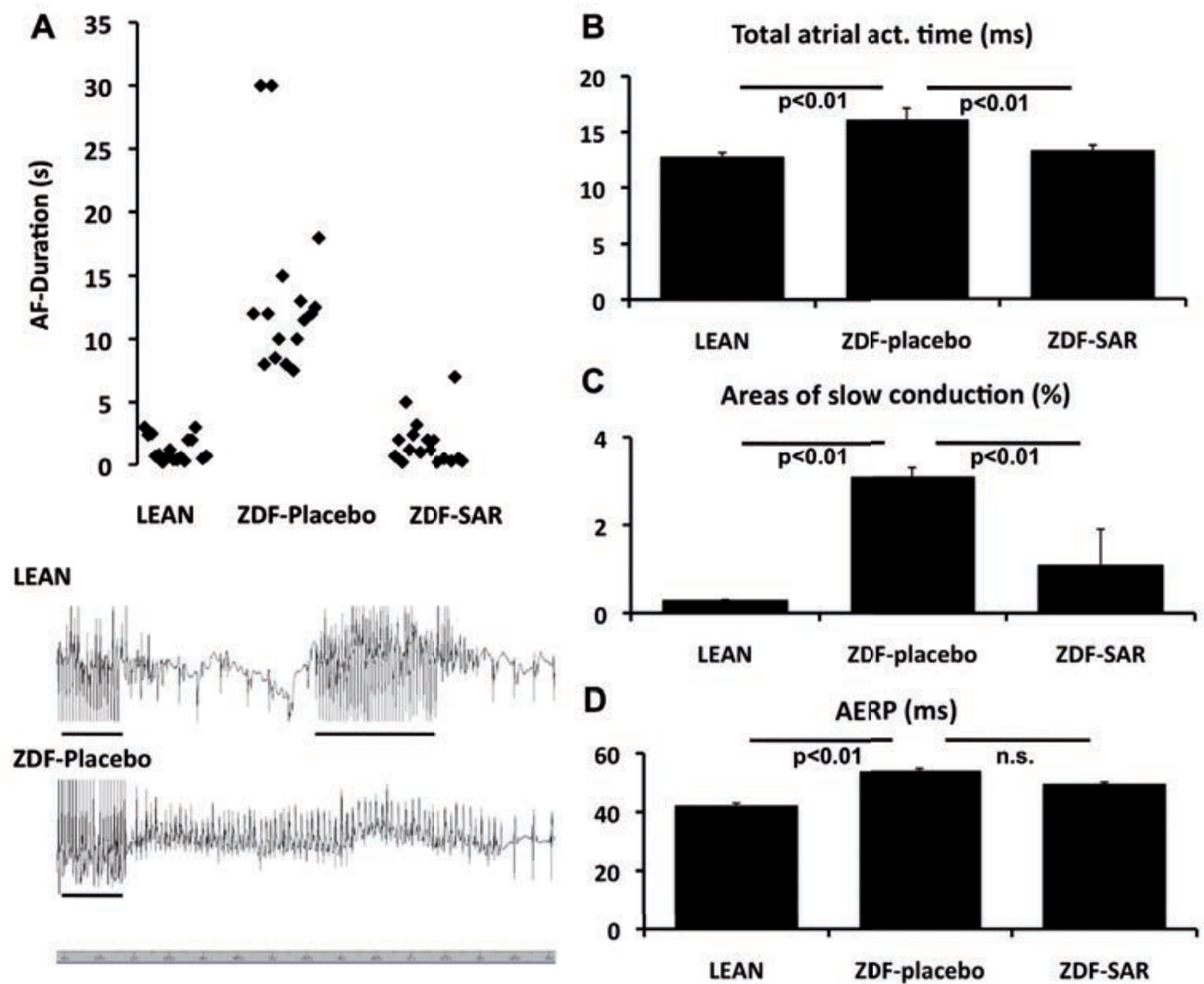

Figure 2: Atrial electrophysiological measurements in rats. (A) AF-duration induced by burst-pacing (top) and representative atrial electrograms in ZDF-placebo and in LEAN (bottom). (B) Total atrial activation time, (C) areas of slow conduction and (D) left atrial effective refractory period (AERP) at a stimulation frequency of 400/min (basic cycle lengths (BCL): 150ms) are shown at the right. 
A
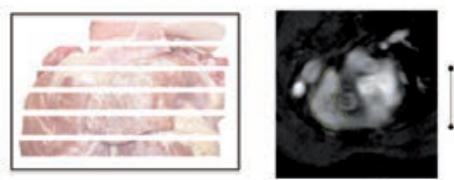

B

LA-volume/time curves:
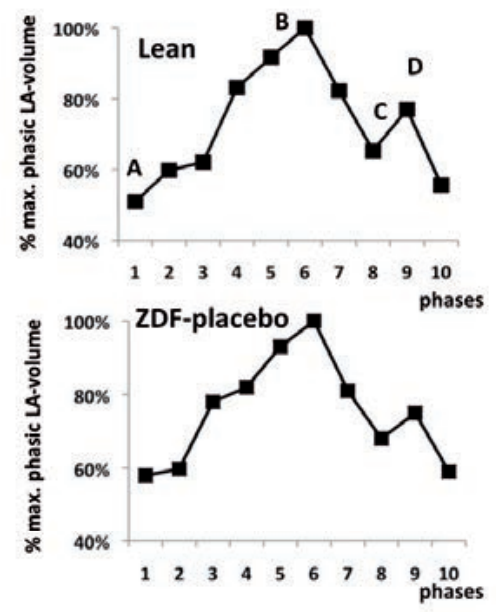

C

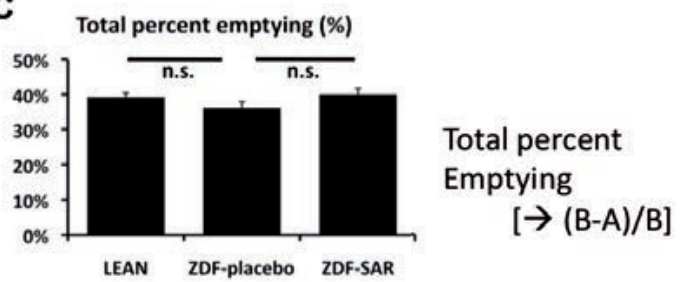

D Passive percent emptying (\%)

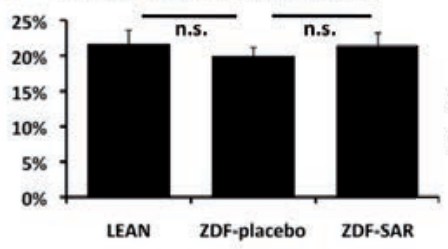

Passive percent Emptying $[\rightarrow(B-C) / B]$

E Active percent emptying (\%)

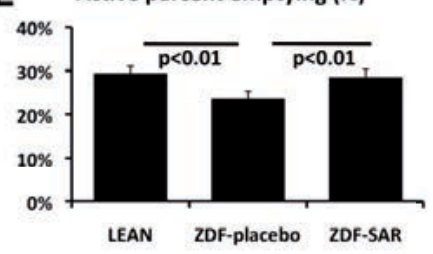

Active percent Emptying $[\rightarrow(D-A) / D]$

Figure 3: Atrial emptying function in rats. (A) Representative makroskopic and MRI-images and (B) LA-volume/time curves showing the \% maximal phasic LAvolume change in LEAN and in ZDF-placebo. Cardiac cycle is divided in 10 phases. (A: minimal LA-volume, B: maximal LA-volume, C: minimal volume at the end of rapid passive emptying, D: max volume before active emptying.) (C) Total percent emptying, (D) passive percent emptying and (E) active percent emptying determined out of the LA-volume/time curve are shown at the right. 


\section{LA-structural remodeling}

In sirius red-stained sections, the degree of atrial tissue fibrosis was increased in ZDF-placebo LA when compared to LEAN (shown in Figure 4) as were myocyte diameters and myocyte-myocyte distances within bundles as a measure of enhanced extracellular LA-matrix formation. SAR-treatment in ZDF-rats prevented the increase in myocyte-myocyte distances and total atrial tissue fibrosis but did not modify increased cell-diameter in ZDF-rats.

SAR reduced LA-gene expression of the profibrotic and proinflammatory adhesion protein osteopontin, which was highly upregulated in ZDF-placebo when compared to LEAN (Table 2). Osteopontin plasma concentration was significantly reduced in ZDF-SAR $(8.4 \pm 0.4 \mathrm{ng} / \mathrm{ml}, \mathrm{p}<0.01)$ when compared to ZDF-placebo $(12.1 \pm 1.4 \mathrm{ng} / \mathrm{ml})$. Other profibrotic markers like TGFB1 and MMP2 and Col1a1 gene expression were clearly detectable but kept unchanged on mRNA level in ZDF-placebo or ZDF-SAR.

\section{Connexin 43}

In LEAN we found the typical intracellular distribution of $\mathrm{Cx} 43$, with a clear accentuation of $\mathrm{Cx} 43$ at the cell poles, and nearly no $\mathrm{C} \times 43$ at the lateral sides of the cells. However, in ZDF-placebo rats there was a significantly enhanced lateral Cx43 immunostaining, whereas polar staining remained unchanged. This lateralization of Cx43 was significantly attenuated by SAR. In figure 5, the pattern of Cx43 immunostaining in LA-tissue is shown. 


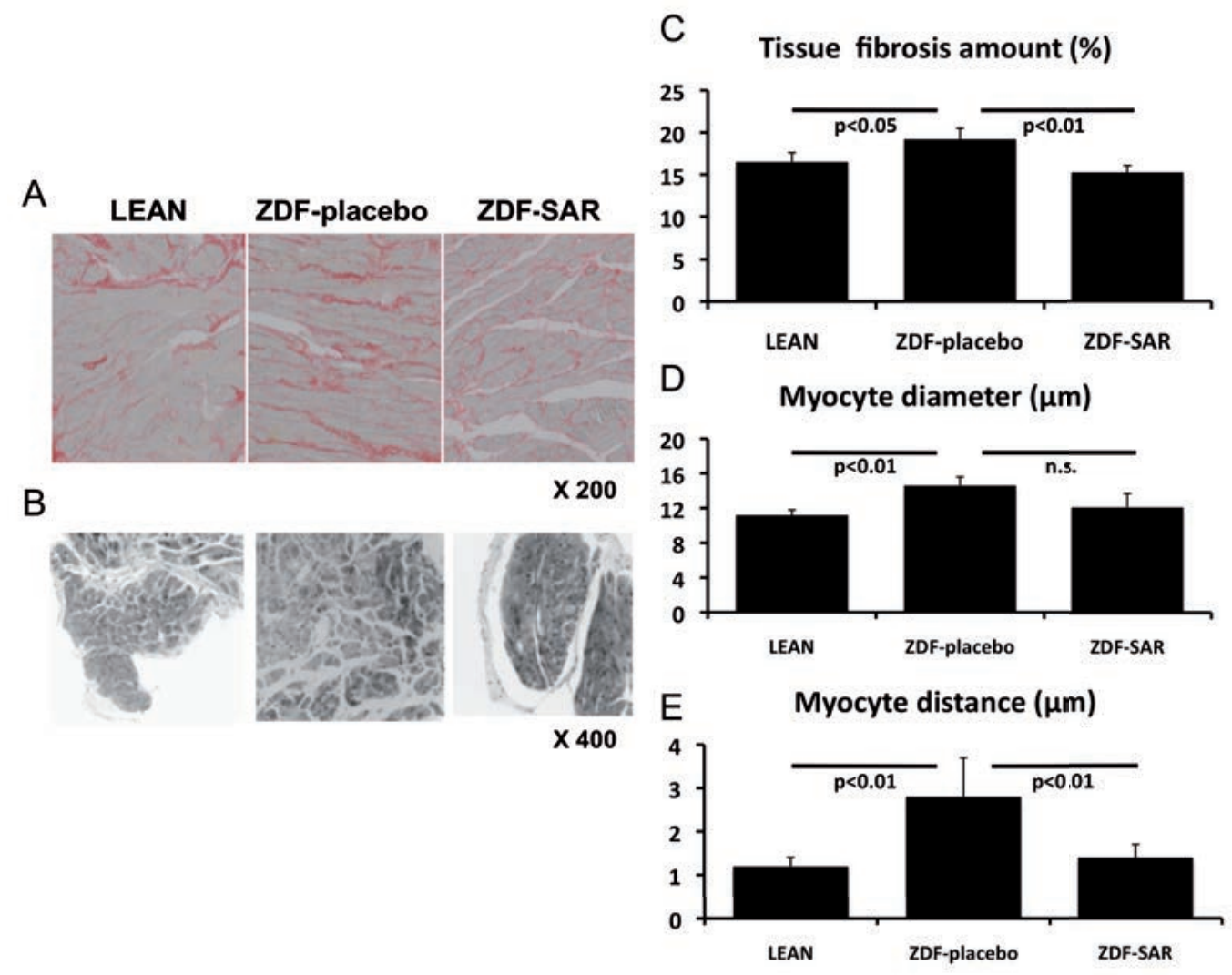

Figure 4: Atria histological analysis. Representative histological images of (A) sirius red stained and (B) H\&E stained left atrial preperations in LEAN, ZDF-placebo and ZDF-SAR. Results for (C) tissue fibrosis amount, (D) myocyte diameter and (E) myocyte distances are shown at the right. 

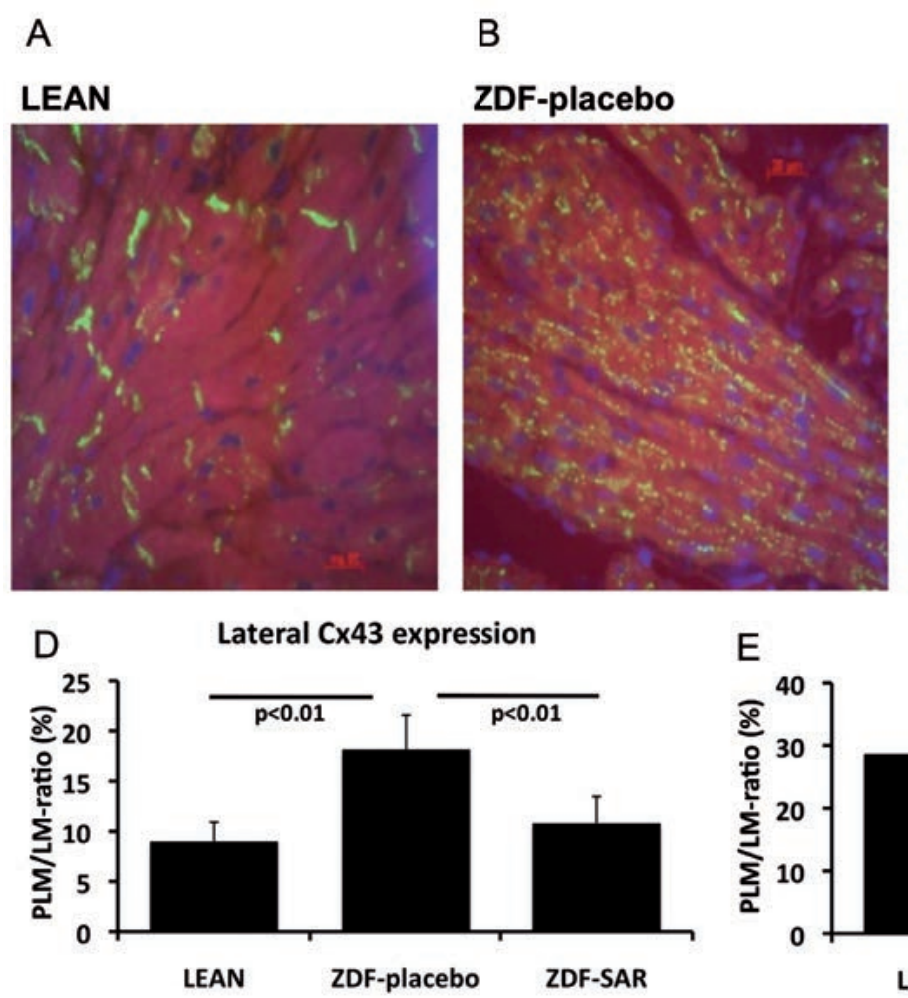

C

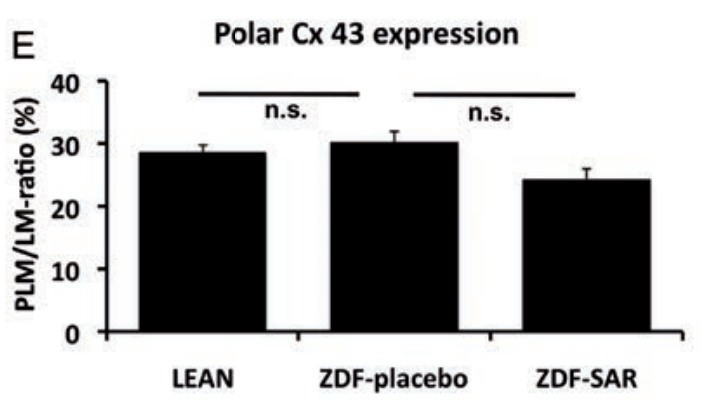

Figure 5: Atrial connexin 43 expression. Representative immunohistochemical staining for $\mathrm{Cx} 43$ in left atrial preperations in (A) LEAN, (B) ZDF-placebo and (C) ZDF-SAR. Quantification of positively stained (D) lateral and (E) polar cardiomyocyte membrane. 
Components of the renin-angiotensin system and bradykinin-degrading activity

LA-total bradykinin-degrading activity was increased in ZDF-placebo compared to LEAN and reduced to LEAN values in ZDF-SAR (Figure 6A). LA-tissue angiotensin II levels were neither altered in ZDF nor in SAR-treated ZDF when compared to LEAN (Figure 6B). Serum ACE-activity was slightly higher in both, ZDF-placebo (107.1 \pm 2.1 $\mathrm{U} / \mathrm{l}, \mathrm{p}<0.01)$ and ZDF-SAR rats $(109.1 \pm 1.5 \mathrm{U} / \mathrm{l}, \mathrm{p}<0.01)$ when compared to LEAN (90.3 $\pm 0.9 \mathrm{U} / \mathrm{l}, \mathrm{p}<0.01)$. In LA-tissue, ACE, chymase or ATRa and ATRb mRNA expression were unchanged in ZDF-placebo with no effect of SAR treatment (Table 2).

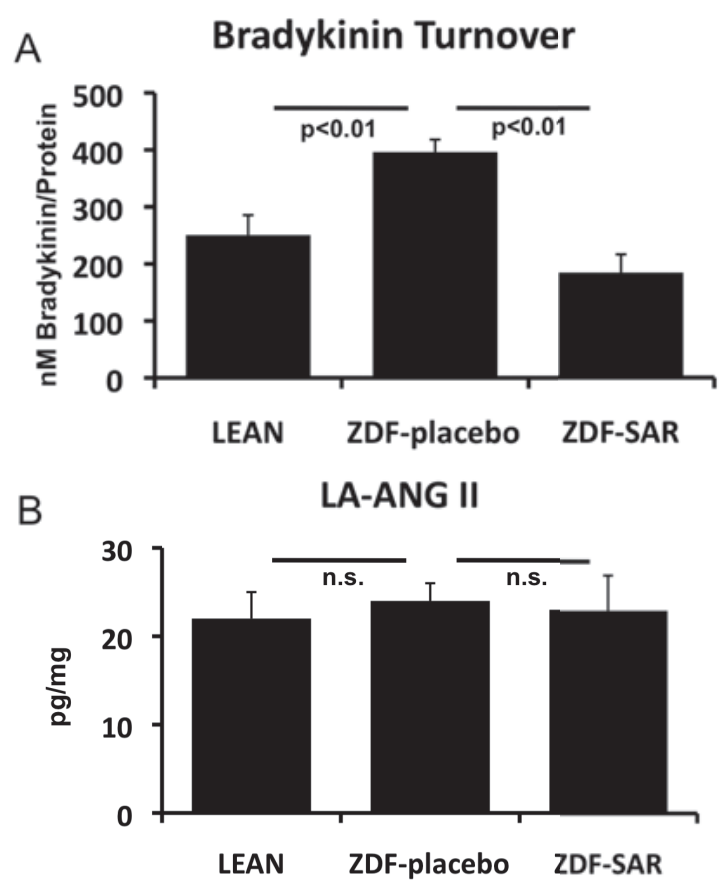

Figure 6: (A) Total bradykinin turnover and (B) angiotensine II content of left atrial tissue in LEAN, ZDF-placebo and ZDF-SAR. 
Table 2: Left atrial gene expression data

\begin{tabular}{lccc}
\multicolumn{2}{c}{ LEAN } & ZDF-placebo & ZDF-SAR \\
\hline $\begin{array}{l}\text { Gene } \\
\text { expression/GAPDH }\end{array}$ & mean [\%of mean & mean [\%of mean & mean [\%of mean \\
Markers of the renin-angiotensin system & ZDF] & ZDF] \\
ACE & $82 \pm 10$ & $100 \pm 9$ & $98 \pm 13$ \\
Agtr 1a & $91 \pm 15$ & $100 \pm 18$ & $85 \pm 13$ \\
Agtr1b & $68 \pm 11$ & $100 \pm 35$ & $99 \pm 37$ \\
Chymase & $89 \pm 41$ & $100 \pm 36$ & $82 \pm 26$
\end{tabular}

Markers of LA-structural remodeling

\begin{tabular}{lccc} 
Osteopontin & $5 \pm 2$ & $100 \pm 40^{+}$ & $54 \pm 12 *^{+}$ \\
TGFß1 & $67 \pm 2$ & $100 \pm 14$ & $86 \pm 21$ \\
Colla1 & $91 \pm 15$ & $100 \pm 18$ & $83 \pm 13$ \\
MMP2 & $98 \pm 12$ & $100 \pm 11$ & $97 \pm 15$ \\
TIMP & $18 \pm 5$ & $100 \pm 37^{+}$ & $54 \pm 12$ \\
\hline
\end{tabular}

${ }^{*} p<0.01$ vs. ZDF-placebo, ${ }^{+} p<0.01$ vs. LEAN 


\section{Discussion}

In ZDF-rats, a rat model for T2D, susceptibility to AT/AF was increased and LAemptying function parameters were impaired while LV systolic function remained unchanged. These findings were accompanied by upregulation of the lysosomal enzyme CathA in LA. Inhibition of CathA by SAR attenuated arrhythmogenic and functional atrial remodeling without influencing T2D-related metabolic changes, blood pressure, LV-systolic function, plasma ACE-activity or LA-angiotensin II levels in ZDF. CathA-inhibition reduced serum BNP-levels, LA-gene expression of profibrotic and proinflammatory factor osteopontin, and LA-bradykinin-degrading activity.

\section{The proarrhythmic substrate for AF in ZDF-rats}

In ZDF, increased susceptibility to AT/AF was associated with longer total atrial activation times, more areas of local atrial conduction slowing and prolonged AERP when compared to LEAN. Local heterogeneities in conduction have been demonstrated to underlie the high AF susceptibility in structurally remodeled atria in a variety of animal models. ${ }^{19,23}$ In ZDF-rats, myocyte-myocyte distances in the atrial myocardium as a measure of enhanced extracellular matrix formation were increased as were myocyte diameters. Additionally, enhanced ANP expression in LA of ZDF may contribute to atrial amyloidosis. ${ }^{25,26} \mathrm{Cx} 43$ lateralization was more pronounced in atria of old ZDF-rats compared to LEAN, a finding also reported in patients with $\mathrm{AF}$ and various animal models for $\mathrm{AF}^{22,27,28}$ These structural atrial changes together with the disorganization of connexins likely resulted in disruption of side to side electrical connections between muscle bundles obviously accounting for the impaired atrial conduction properties and increased susceptibility to AT/AF observed in old ZDF-rats compared to LEAN. Interestingly, this arrhythmogenic 
substrate and these structural alterations of the extracellular matrix occurred without any changes in LA-dimensions in ZDF-rats. In contrast, prolongation of atrial refractoriness in ZDF-rats suggests that ion channel remodeling, as seen in tachycardia-induced atrial remodeling ${ }^{6}$, does not play an important role in the enhanced susceptibility for AT/AF in ZDF-rats.

\section{Atrial contractile dysfunction in ZDF-rats}

Experimental and clinical information about changes in ventricular function caused by pure diabetic conditions are controversially discussed..$^{29,30,31}$ In our study, ZDF rats showed moderate signs for diastolic ventricular dysfunction with fully preserved systolic function. Additionally, we found an impairment of active LA-emptying function in ZDF-rats suggestive for a LA-hypocontractility in ZDF-rats. Those findings are in line with impairment of LA-emptying function revealed by echocardiography in patients with type 1 diabetes and preserved LV-function. ${ }^{32}$ Potentially, impaired LAemptying function reduces exercise tolerance in diabetic patients. ${ }^{7,8,9}$ Besides, it might contribute to the enhanced risk for strokes in patients with T2D. ${ }^{10}$ Atrial contractile dysfunction reduces local blood flow velocity increasing the risk for thrombus formation. The mechanisms leading to impaired LA-emptying function in diabetes are unknown. A similar reduction of contractile function with unchanged or prolonged AERP was found in a dog model with congestive heart failure ${ }^{33}$ and in goats with dilated atria. ${ }^{34}$ Increased interstitial fibrosis amount in old ZDF-rats could account for abnormal myocardial stiffness and ultimately for atrial dysfunction. Another possibility is altered energetics of myofilaments as also found in patients with $\mathrm{AF}^{35}$ 


\section{Role of CathA in atrial remodeling in ZDF-rats}

In this report, we identify a relevant role of CathA for the LA-remodeling process in T2D. LA-CathA activity was increased in ZDF compared to LEAN. The novel CathA inhibitor SAR attenuated functional and arrhythmogenic atrial remodeling characterized by increased tissue fibrosis, myocyte-myocyte distances and lateralization of $\mathrm{Cx} 43$. Smaller myocyte-myocyte distances and attenuation of lateralization of $\mathrm{Cx} 43$ increase coupling between the atrial bundle network and are likely involved in the reduction of AF/AT-duration and conduction disturbances in ZDF-SAR compared to ZDF-placebo. As there were no changes in atrial refractoriness related to treatment with SAR an effect of the compound on ion channel composition does not likely contribute to the protective effect of SAR.

LA-gene expression and plasma concentration of the profibrotic and proinflammatory marker osteopontin (OPN) was markedly increased in ZDF-rats. CathA-inhibition by SAR was associated with a reduction in LA-gene expression and plasma concentration of OPN. OPN is a secreted multifunctional phosphoprotein, which plays an important role in the pathogenesis and fibrosis formation and inflammation in CHF and diabetic cardiopathy. ${ }^{36}$ Its expression is accelerated by mechanical stress including pressure/volume loading and angiotensin II infusion in rats. ${ }^{37,38}$ OPN knockout prevented diastolic dysfunction and interstitial fibrosis following myocardial infarction. ${ }^{39}$ Additionally, streptozotocin-induced diabetic cardiomyopathy and cardiac fibrosis were attenuated in OPN-deficient mice, suggesting that OPN is a key factor for the remodeling process in the heart especially under diabetic conditions. ${ }^{40}$ Both, CathA and OPN are involved in inflammation occurring rapidly (within 24h) after onset of ventricular tachypacing in dogs. However, these inflammatory changes are transient and disappear gradually. ${ }^{41}$ In line with this, we did not observe indicators for enhanced inflammation in ZDF rats. However, inflammatory processes 
might well have contributed to extracellular matrix changes at an early stage of T2D in ZDF. Thus, inhibition of OPN expression by SAR may be of value in the treatment and prevention of diabetic atrial cardiomyopathy.

We found arguments to believe that the action of SAR differs substantially from inhibitors involved in the renin-angiotensin system. First, atrial tissue angiotensin II concentration and gene expression of different players of the local renin-angiotensin system were not modified by SAR, suggesting a mode of action different to simple modulation of local renin-angiotensin system. Furthermore, unlike established targets in the cardiac renin-angiotensin and kinin system like angiotensin converting enzyme, CathA is only weakly expressed in the vasculature and has an acidic $\mathrm{pH}$ optimum of activity. ${ }^{11}$ Besides, angiotensin I and bradykinin have been described as substrates for CathA in vitro. ${ }^{11}$ CathA-inhibition by SAR resulted in a pronounced decrease in bradykinin-degrading activity in LA-homogenates which may well explain the observed atrial protective effects of CathA-inhibtion in ZDF-rats. Bradykinin elicits positive inotropic effects in isolated LA-preparation of the guinea pig heart, which could explain the attenuation of LA-emptying function in SAR-treated ZDF. ${ }^{42}$ Also, increase of bradykinin has been shown to attenuate cardiac hypertrophy and fibrosis. ${ }^{43}$ Thus, reduction of bradykinin turnover might well explain the beneficial effects of the CathA inhibitor. 


\section{Limitations}

We investigated the effect of SAR in ZDF-rats at one specific time point at the end of the study. The exact time course of the beneficial effects of SAR treatment thus remains to be studied. We did not investigate whether the compound tested is able to reverse preexisting structural and functional atrial remodeling. Further intensive studies are necessary to identify the full pathway involved in the beneficial effect of SAR in diabetic conditions.

\section{Conclusion}

CathA gene expression and activity in LA were increased in old ZDF rats. CathAinhibition attenuated increased myocyte-myocyte-distances and lateralization of Cx43. CathA-inhibition decreased bradykinin-degrading activity in LA-homogenates and attenuated elevated LA-gene expression and plasma level of profibrotic and proinflammatory marker OPN which might explain the protective effect of SAR. This was associated with reduced susceptibility to AF and improved LA-emptying function parameters. The atrial protective effect of CathA-inhibition was neither associated with changes in markers of the renin-angiotensin system, blood pressure, ventricular or renal function nor with changes in LA-dimensions. These findings provide evidence for CathA playing a role for cardiac and especially atrial remodeling in T2D and emphasize the potential use of CathA-inhibitors for atrial upstream therapy in patients with T2D. 


\section{References}

1. Kannel WB, Wolf PA, Benjamin EJ, Levy D. Prevalence, incidence, prognosis, and predisposing conditions for atrial fibrillation: population-based estimates. Am J Cardiol. 1998;82:2N-9N.

2. Brand FN, Abbott RD, Kannel WB, Wolf PA. Characteristics and prognosis of lone atrial fibrillation. 30-year follow-up in the Framingham Study. JAMA. 1985;254:3449-3453.

3. Movahed MR, Hashemzadeh M, Jamal MM. Diabetes mellitus is a strong, independent risk for atrial fibrillation and flutter in addition to other cardiovascular disease. Int J Cardiol. 2005;105:315-318.

4. Ostgren CJ, Merlo J, Rastam L, Lindblad U. Atrial fibrillation and its association with type 2 diabetes and hypertension in a Swedish community. Diabetes Obes Metab. 2004;6:367-374.

5. Kato T, Yamashita T, Sekiguchi A, Sagara K, Takamura M, Takata S, Kaneko S, Aizawa T, Fu LT. What are arrhythmogenic substrates in diabetic rat atria? J Cardiovasc Electrophysiol. 2006;17:890-894.

6. Schotten U, Verheule S, Kirchhof P, Goette A. Pathophysiological mechanisms of atrial fibrillation: a translational appraisal. Physiol Rev. $2011 ; 91: 265-325$.

7 Regensteiner JG, Sippel J, McFarling ET, Wolfel EE, Hiatt WR. Effects of non-insulin-dependent diabetes on oxygen consumption during treadmill exercise. Med Sci Sports Exerc. 1995;27:875-881. 
8. Regensteiner JG, Bauer TA, Reusch JE, Brandenburg SL, Sippel JM, Vogelsong AM, Smith S, Wolfel EE, Eckel RH, Hiatt WR. Abnormal oxygen uptake kinetic responses in women with type II diabetes mellitus. J Appl Physiol. 1998;85:310-317.

9. Wei M, Gibbons LW, Mitchell TL, Kampert JB, Lee CD, Blair SN. The association between cardiorespiratory fitness and impaired fasting glucose and type 2 diabetes mellitus in men. Ann Intern Med. 1999;130:89-96.

10. Wolf PA, Abbott RD, Kannel WB. Atrial fibrillation as an independent risk factor for stroke: the Framingham Study. Stroke. 1991;22:983-988.

11. Hiraiwa M. Cathepsin A/protective protein: an unusual lysosomal multifunctional protein. Cell Mol Life Sci. 1999;56:894-907.

12. Jackman HL, Massad MG, Sekosan M, Tan F, Brovkovych V, Marcic BM, Erdos EG. Angiotensin 1-9 and 1-7 release in human heart: role of cathepsin A. Hypertension. 2002;39:976-981.

13. Urata H, Hoffmann S, Ganten D. Tissue angiotensin II system in the human heart. Eur Heart J. 1994;15 Suppl D:68-78.

14. Urata H, Ganten D. Cardiac angiotensin II formation: the angiotensin-I converting enzyme and human chymase. Eur Heart J. 1993;14 Suppl I:177182.

15. Steckelings UM, Rompe F, Kaschina E, Unger T. The evolving story of the RAAS in hypertension, diabetes and CV disease: moving from macrovascular to microvascular targets. Fundam Clin Pharmacol. 2009;23:693-703. 
16. Ito H, Majima M, Nakajima S, Hayashi I, Katori M, Izumi T. Effect of prolonged administration of a urinary kinase inhibitor, ebelactone B on the development of deoxycorticosterone acetate-salt hypertension in rats. $\mathrm{Br} \mathrm{J}$ Pharmacol. 1999;126:613-620.

17. Hayashi I, Majima M, Fujita T, Okumura T, Kumagai Y, Tomita N, Morishita R, Higaki J, Ogiwara T. In vivo transfer of antisense oligonucleotide against urinary kininase blunts deoxycorticosterone acetate-salt hypertension in rats. Br J Pharmacol. 2000;131:820-826.

18. Ruf S, Buning C, Schreuder H, Horstick G, Linz W, Olpp T, Pernerstorfer J, Hiss K, Kroll K, Kannt A, Kohlmann M, Linz D, Hübschle T, Rütten H, Wirth K, Schmidt T, Sadowski T. Novel B-amino acid derivatives as inhibitors of cathepsin A. J Med Chem. 2012;55:7636-7649.

19. Neuberger HR, Schotten U, Verheule S, Eijsbouts S, Blaauw Y, van Hunnik A, Allessie M. Development of a substrate of atrial fibrillation during chronic atrioventricular block in the goat. Circulation. 2005;111:30-37.

20. Jarvinen V, Kupari M, Hekali P, Poutanen VP. Assessment of left atrial volumes and phasic function using cine magnetic resonance imaging in normal subjects. Am J Cardiol. 1994;73:1135-1138.

21. Nori D, Raff G, Gupta V, Gentry R, Boura J, Haines DE. Cardiac magnetic resonance imaging assessment of regional and global left atrial function before and after catheter ablation for atrial fibrillation. $J$ Interv Card Electrophysiol. 2009;26:109-117.

22. Polontchouk L, Haefliger JA, Ebelt B, Schaefer T, Stuhlmann D, Mehlhorn U, Kuhn-Regnier F, De Vivie ER, Dhein S. Effects of chronic atrial fibrillation on 
gap junction distribution in human and rat atria. J Am Coll Cardiol. 2001;38:883-891.

23. Li D, Fareh S, Leung TK, Nattel S. Promotion of atrial fibrillation by heart failure in dogs: atrial remodeling of a different sort. Circulation. 1999;100:8795.

24. Satoh T, Zipes DP. Cesium-induced atrial tachycardia degenerating into atrial fibrillation in dogs: atrial torsades de pointes? J Cardiovasc Electrophysiol. 1998;9:970-975.

25. Rocken C, Peters B, Juenemann G, Saeger W, Klein HU, Huth C, Roessner A, Goette A. Atrial amyloidosis: an arrhythmogenic substrate for persistent atrial fibrillation. Circulation. 2002;106:2091-2097.

26. Takahashi M, Hoshii Y, Kawano H, Gondo T, Yokota T, Okabayashi H, Shimada I, Ishihara T. Ultrastructural evidence for the formation of amyloid fibrils within cardiomyocytes in isolated atrial amyloid. Amyloid. 1998;5:35-42.

27. Rucker-Martin C, Milliez P, Tan S, Decrouy X, Recouvreur M, Vranckx R, Delcayre C, Renaud JF, Dunia I, Segretain D, Hatem SN. Chronic hemodynamic overload of the atria is an important factor for gap junction remodeling in human and rat hearts. Cardiovasc Res. 2006;72:69-79.

28. Burstein B, Comtois P, Michael G, Nishida K, Villeneuve L, Yeh YH, Nattel S. Changes in connexin expression and the atrial fibrillation substrate in congestive heart failure. Circ Res. 2009 4;105:1213-1222.

29. Cosson S, Kevorkian JP. Left ventricular diastolic dysfunction: an early sign of diabetic cardiomyopathy? Diabetes Metab. 2003;29:455-466.

30. Schafer S, Huber J, Wihler C, Rutten H, Busch AE, Linz W. Impaired left 
ventricular relaxation in type 2 diabetic rats is related to myocardial accumulation of N(epsilon)-(carboxymethyl) lysine. Eur J Heart Fail. 2006;8:26.

31. Daniels A, Linz D, van Bilsen M, Rütten H, Sadowski T, Ruf S, Juretschke HP, Neumann-Haefelin C, Munts C, van der Vusse GJ, van Nieuwenhoven FA. Long-term severe diabetes only leads to mild cardiac diastolic dysfunction in Zucker diabetic fatty rats. Eur J Heart Fail. 2012;14:193-201.

32. Acar G, Akcay A, Sokmen A, Ozkaya M, Guler E, Sokmen G, Kaya H, Nacar AB, Tuncer C. Assessment of atrial electromechanical delay, diastolic functions, and left atrial mechanical functions in patients with type 1 diabetes mellitus. J Am Soc Echocardiogr. 2009;22:732-738.

33. Wakili R, Yeh YH, Yan Qi X, Greiser M, Chartier D, Nishida K, Maguy A, Villeneuve LR, Boknik P, Voigt N, Krysiak J, Kääb S, Ravens U, Linke WA, Stienen GJ, Shi Y, Tardif JC, Schotten U, Dobrev D, Nattel S. Multiple potential molecular contributors to atrial hypocontractility caused by atrial tachycardia remodeling in dogs. Circ Arrhythm Electrophysiol. 2010;3(5):53041.

34. Greiser M, Neuberger HR, Harks E, El-Armouche A, Boknik P, de Haan S, Verheyen F, Verheule S, Schmitz W, Ravens U, Nattel S, Allessie MA, Dobrev D, Schotten U. Distinct contractile and molecular differences between two goat models of atrial dysfunction: AV block-induced atrial dilatation and atrial fibrillation. J Mol Cell Cardiol. 2009;46:385-394. 
35. Mihm MJ, Yu F, Carnes CA, Reiser PJ, McCarthy PM, Van Wagoner DR, Bauer JA. Impaired myofibrillar energetics and oxidative injury during human atrial fibrillation. Circulation. 2001;104:174-180.

36. Okamoto H. Osteopontin and cardiovascular system. Mol Cell Biochem. $2007 ; 300: 1-7$

37. Xie Z, Singh M, Singh K. Osteopontin modulates myocardial hypertrophy in response to chronic pressure overload in mice. Hypertension. 2004;44:826831.

38. Ashizawa N, Graf K, Do YS, Nunohiro T, Giachelli CM, Meehan WP, Tuan TL, Hsueh WA. Osteopontin is produced by rat cardiac fibroblasts and mediates A(II)-induced DNA synthesis and collagen gel contraction. J Clin Invest. 1996;98:2218-2227.

39. Trueblood NA, Xie Z, Communal C, Sam F, Ngoy S, Liaw L, Jenkins AW, Wang J, Sawyer DB, Bing OH, Apstein CS, Colucci WS, Singh K. Exaggerated left ventricular dilation and reduced collagen deposition after myocardial infarction in mice lacking osteopontin. Circ Res. 2001;88:10801087.

40. Subramanian V, Krishnamurthy P, Singh K, Singh M. Lack of osteopontin improves cardiac function in streptozotocin-induced diabetic mice. Am J Physiol Heart Circ Physiol. 2007;292:H673-83.

41. Hanna N, Cardin S, Leung TK, Nattel S. Differences in atrial versus ventricular remodeling in dogs with ventricular tachypacing-induced congestive heart failure. Cardiovasc Res. 2004;63:236-244. 
42. Erdos EG, Jackman HL, Brovkovych V, Tan F, Deddish PA. Products of angiotensin I hydrolysis by human cardiac enzymes potentiate bradykinin. $\mathrm{J}$ Mol Cell Cardiol. 2002;34:1569-1576.

43. Linz W, Wiemer G, Gohlke P, Unger T, Schölkens BA. Contribution of kinins to the cardiovascular actions of angiotensin-converting enzyme inhibitors. Pharmacol Rev. 1995;47:25-49. 
Chapter 3

Negative Tracheal Pressure During Obstructive Respiratory Events Promotes Atrial Fibrillation by Vagal Activation.

Dominik Linz; Hans-Ruprecht Neuberger; Michael Böhm; Klaus Wirth; Ulrich Schotten.

Heart Rhythm. 2011 Sep;8(9):1436-43. 


\section{Abstract}

Introduction: Obstructive sleep apnea (OSA) causes negative tracheal pressure (NTP) and is associated with atrial fibrillation (AF). This study aimed to determine the mechanism of atrial electrophysiological changes during tracheal occlusion with or without applied NTP and to evaluate the role of vagal activation, $\mathrm{Na}^{+} / \mathrm{H}^{+}$-exchanger (NHE) and ATP-dependent potassium channels ( $\left.\mathrm{K}_{\mathrm{ATP}}\right)$.

Methods: 17 closed-chest pigs were anesthetized with urethane and an endotracheal tube was placed to apply NTP (up to -100 mbar) comparable to clinically observed OSA in patients by a negative pressure device for a time period of 2 minutes. Right atrial refractory periods (AERP) and AF-inducibility were measured transvenously by a monophasic action potential recording and stimulation catheter.

Results: All tracheal occlusions with and without applied NTP resulted in comparable increases in blood pressure and hypoxemia. NTP shortened AERP (157.0 $\pm 2.8 \mathrm{~ms}$ to $102.1 \pm 6.2 \mathrm{~ms}, \mathrm{p}<0.0001)$ and enhanced AF-inducibility during AERP-measurements from $0 \%$ at baseline to $90 \%(p<0.00001)$ during NTP. Release of NTP resulted in a prompt restoration of sinus rhythm and AERP returned to normal. NTP-induced AERP-shortening and AF-inducibility were prevented by atropine or vagotomy. Neither the NHE-blocker cariporide nor the $\mathrm{K}_{\mathrm{ATP}}$-channelblocker glibenclamide abolished NTP-induced AERP-shortening. By contrast, tracheal occlusion without applied NTP caused comparable changes in blood gases but did not induce AERP-shortening or AF-inducibility.

Conclusions: NTP during obstructive events is a strong trigger for AF compared to changes in blood gases alone. NTP caused AERP-shortening and increased susceptibility to AF mainly by enhanced vagal activation. AERP-shortening was not prevented by $\mathrm{K}_{\text {ATP-channel-blockade or NHE-blockade. }}$ 


\section{Introduction}

Patients with obstructive sleep apnea (OSA) show a high prevalence of atrial fibrillation (AF) ranging from 32 to $49 \%{ }^{1-3}$. OSA was the strongest predictor of recurrent $\mathrm{AF}$ following catheter ablation ${ }^{4}$ and patients with untreated OSA have a higher risk of recurrence of $\mathrm{AF}$ after successful cardioversion ${ }^{5}$. The finding that appropriate treatment of OSA with continuous positive airway pressure (CPAP) is associated with lower recurrence of AF suggests a causal association of OSA inducing $A F^{6}$. Several mechanisms causing $A F$ in OSA have been discussed including hypoxemia and acidosis. These changes in blood gases might influence the parasympathetic and sympathetic tone leading to a substrate for AF. While enhanced vagal tone is known to induce AERP-shortening, increased sympathetic tone and autonomic dysbalance may enhance spontaneous triggered activity that could induce $\mathrm{AF}^{7}$. Severe bradycardia and atrioventricular block are frequently seen in OSA and suggest vagal activation ${ }^{8}$. However, other mediators beyond hypoxemia might be relevant in humans. Obstructive apneas are caused by collapse of the upper airway during sleep resulting in repetitive forced inspiration against the obstructed upper airway. Correspondingly substantial negative changes in intratracheal pressure down to -100 mbar were observed in OSA patients ${ }^{3,9,10}$. The resulting intrathoracic pressure changes might stretch heart chambers and lead to changes in transmural pressure gradients particularly in the thin walled atria. The influence of negative tracheal pressure (NTP) on atrial electrophysiology has not been investigated, yet. We hypothesized that the NTP during obstructive events and not changes in blood gases is responsible for increased AF-susceptibility in OSA. Therefore, we investigated electrophysiological changes in the pig right atrium during airway obstructions with or without NTP generated by a negative pressure device. Atropine followed by vagotomy was used to study the role of vagal activation. 
Additionally, we characterized the role of hypoxia and stretch activated $\mathrm{Na}^{+} / \mathrm{H}^{+}$exchanger subtype 1 (NHE) and ATP-dependent potassium channel $\left(\mathrm{K}_{\text {ATP }}\right)^{11-13}$ by cariporide and glibenclamide.

\section{Methods}

All animal studies were performed in accordance to the German law for the protection of animals. Furthermore, the investigation conforms with the guide for the Care and Use of laboratory Animals published by the US National Institutes of Health (NIH Publication No. 85-23, revised 1996).

\section{Experimental model for OSA}

In 17 chest-closed male castrated pigs $(25-30 \mathrm{~kg})$ of the German Landrace (anesthetized with $20 \%$ urethane $(0.8 \mathrm{ml} / \mathrm{kg}$ i.v. load, $0.4 \mathrm{ml} / \mathrm{kg} / \mathrm{h}$ maintainance) and $4 \%$ alpha-chloralose ( $0.4 \mathrm{ml} / \mathrm{kg}$ i.v. load, $0.1 \mathrm{ml} / \mathrm{kg} / \mathrm{h}$ maintainance)), a tracheotomy was performed to place an endotracheal tube. This tube was used for tracheal occlusion and to apply different levels of negative tracheal pressures by a negative pressure device. The negative pressure device consisted of a negative pressure container $(50 \mathrm{~L})$ and a vacuum pump controlled by a manometer. The tracheal tube was connected to the vacuum container and a solenoid valve was opened and the tracheal tube and the vacuum container created a closed system. In pilot experiments, spontaneous breathing attempts during tracheal occlusion without NTP just created fluctuating tracheal pressures down to -40 mbar without causing electrophysiological changes. The Mueller maneuver (forced inspiration against airway obstruction) is used in the clinical setting to simulate conditions, particularly negative thoracic pressure, during obstructive sleep apnea ${ }^{14}$. As a modification of 
this maneuver, we applied NTP at -100 mbar during tracheal occlusion corresponding to NTPs found in patients with OSA ${ }^{3,9,10}$.

Right atrial pressure changes were investigated by a catheter which was advanced via the femoral vein (Gould, P23 series pressure transducer, Hato Rey, Puerto Rico). Blood pressure was measured by a TIP-catheter (Millar PC 350; Millar Instruments, Houston, Texas, USA) in the femoral artery. Temperature was measured via a rectal probe and maintained around $37.0^{\circ} \mathrm{C}$ by a heating lamp. Blood gas analysis $\left(\mathrm{pO}_{2}\right.$, $\mathrm{pCO}_{2}, \mathrm{pH}, \mathrm{O}_{2}$-Saturation) was performed before, at the end and 15 min after release of tracheal occlusion without or with applied NTP in 7 pigs. In all pigs of group 1, the cervical vagi were dissected and bilateral vagotomy was performed.

\section{Experimental design}

In figure 1, the electrophysiological protocol (Fig. 1 A) and the experimental design (Fig. 1 B) are shown. Atrial effective refractory period (AERP) measurements and monophasic action potential (MAP) recordings were performed and AF-inducibility was investigated during normal breathing and at every $30 \mathrm{sec}$ during 2 minutes of either tracheal occlusion without applied NTP or 2 min of tracheal occlusion with applied NTP at -100 mbar. Recovery of the AERP was shown by AERP measurement at 5 and 15 min between the different tracheal occlusion maneuvers. After the first run, vehicle was given to all animals and a second complete run including AERP-measurement, MAP recordings and AF-inducibility during normal breathing and during tracheal occlusion with and without NTP was performed. After those two complete runs at baseline atropine was administered first followed by vagotomy and finally glibenclamide was given (group $1, n=6)$. In group $2(n=5)$, glibenclamide was given first followed by atropine. Three pigs received cariporide followed by atropine (group 3). An additional run of the electrophysiological protocol 
was performed in exactly the same manner 15 min after each drug or bilateral vagotomy. A group of 3 pigs served as time vehicle control (control group).

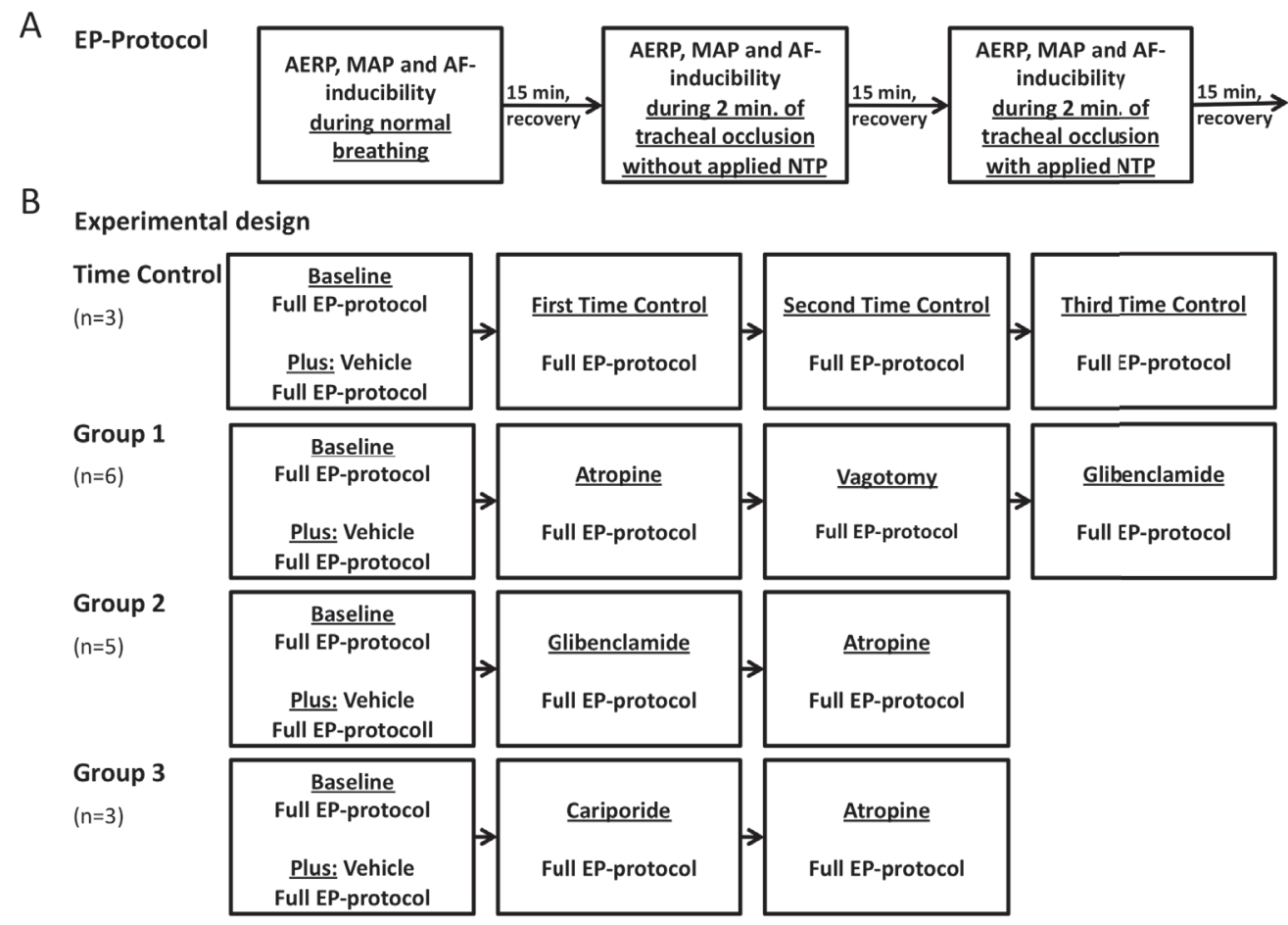

Figure 1: Flow chart of the electrophysiological protocol (EP-protocol): (A) A complete EP-protocol included AERP-measurement, MAP-recording and AFinducibility during normal breathing and every $30 \mathrm{sec}$ during $2 \mathrm{~min}$ of tracheal occlusion without and with applied NTP at $-100 \mathrm{mbar}$ with a recovery period of $15 \mathrm{~min}$ between the different NTP maneuvers. (B) The experimental design is shown. 


\section{Electrophysiological examinations}

Atrial effective refractory period (AERP) measurements at a representative basic cycle length (BCL) of $300 \mathrm{~ms}$ were performed before, during, and after the tracheal occlusion as described previously ${ }^{15}$. Briefly, atrial responses to the pacing procedure were visualized by monophasic action potential (MAP) recordings from the endocardium of the right atrium by a MAP pacing catheter (combined MAP- \& stimulation catheter, 7F, Foehr Medical Instruments GMBH, Seeheim, Germany). The catheter was inserted via a femoral vein. The tip of the catheter was advanced to the lateral right atrium to record a stable and sharp MAP-signal. This catheter was left at one location to ensure AERP determination at a fixed anatomic site throughout the experiment. A conditioning train of 5 basic stimuli (S1) at twice-diastolic pacing threshold was followed by an interpolated extrastimulus (S2, pulse duration $1 \mathrm{~ms}$ ) starting about $30 \mathrm{~ms}$ below the expected AERP with a 5 ms increment (UHS 20, universal heart stimulator; Biotronik, Berlin, Germany). The shortest coupling interval able to elicit a propagated atrial response was taken as the AERP.

For analysis of right atrial MAP-signals, MAP electrode and position of the electrode were the same as used for AERP measurements (see above). A particular effort had to be made to obtain atrial MAPs of sufficient quality. MAP-signals were just used for analysis, if a regular baseline and amplitude of the MAPs could be maintained for at least $30 \mathrm{sec}$ and if the shape of the MAP was stable during NTP-procedures. Right atrial MAP duration was evaluated from $70 \%$ repolarisation during regular pacing (BCL=300ms).

During the AERP-measurement procedure the shortest premature S2-extrastimulus resulting in a propagated response frequently induced episodes of AF. When atrial 
MAP-signals showed an irregular rapid activation (cycle length $<200 \mathrm{~ms}$, duration $>5$ seconds), AF was diagnosed.

Each NTP-maneuver was repeated twice in all animals. The mean percentage of NTP-maneuvers triggering AF was used to describe right atrial vulnerability. After vagotomy, AF-inducibility was additionally tested by 5 times 1 second burst pacing (BCL: 50ms).

\section{Drugs}

Glibenclamide (3mg/kg, synthetized at Sanofi-Aventis) was dissolved in $0.5 \mathrm{ml}$ DMSO and $2.5 \mathrm{ml}$ of polyethyleneglycol (PEG) 400 (Riedel-de Haen, Seelze, Germany). Cariporide (3mg/kg, synthetized at Sanofi-Aventis) was dissolved in saline. All solutions were injected over 5 minutes. Vehicle as a control was injected 15 min before the second baseline NTP-run in all animals.

\section{Statistics}

Data are presented as mean \pm SEM. For comparisons of single repeated measures only, a paired Student's t-test was used. For multiple repeated measures comparisons with the same baseline, repeated-measures ANOVA was used, followed by Dunnet's test to compare individual mean differences if ANOVA was significant. A p value of 0.05 was considered significant. 


\section{Results}

\section{Effect of NTP on hemodynamic parameters and blood gases}

We investigated hemodynamic changes (Fig. 2 A, upper panel) and changes in blood gases (Fig. 2 A, lower panel) during tracheal occlusion with (left) and without (right) applied NTP. During 2 min of tracheal occlusion with applied NTP at 100 mbar, blood pressure did not change significantly and right atrial pressure decreased to $-16.5 \pm 2.5 \mathrm{mmHg}$ vs. $4.3 \pm 0.3 \mathrm{mmHg}$ at baseline $(p<0.001)$. By contrast, atrial pressure did not change significantly during tracheal occlusion without applied NTP. Release of tracheal occlusion resulted in a comparable increase in blood pressure after tracheal occlusion with or without applied NTP. Blood gas analysis during tracheal occlusion revealed significant increases in $\mathrm{pCO}_{2}$, whereas $\mathrm{pO}_{2}(\mathrm{Fig}$. $2 \mathrm{~A}$, lower panel), $\mathrm{SO}_{2}$ (Fig. $2 \mathrm{~B}$ ) and pH (Fig. $2 \mathrm{C}$ ) were decreased. Changes in blood gases did not differ significantly between tracheal occlusion with or without applied NTP. Moderate bradycardia developed during tracheal occlusion with applied NTP (51.4 $\pm 4.1 \mathrm{bpm}$ vs. $79.6 \pm 2.6 \mathrm{bpm}$ before NTP, $\mathrm{p}<0.0001)$ but not during tracheal occlusion without applied NTP (Fig. 2 D). Release of tracheal occlusion resulted in a comparable increase in heart rate after tracheal occlusion with or without applied NTP $(149.9 \pm 17.4 \mathrm{bpm}$ and $150.8 \pm 12.3 \mathrm{bpm}$, respectively, n.s., data not shown). Ten minutes after release of tracheal occlusion, blood gases and hemodynamic parameters returned to normal in all animals. 

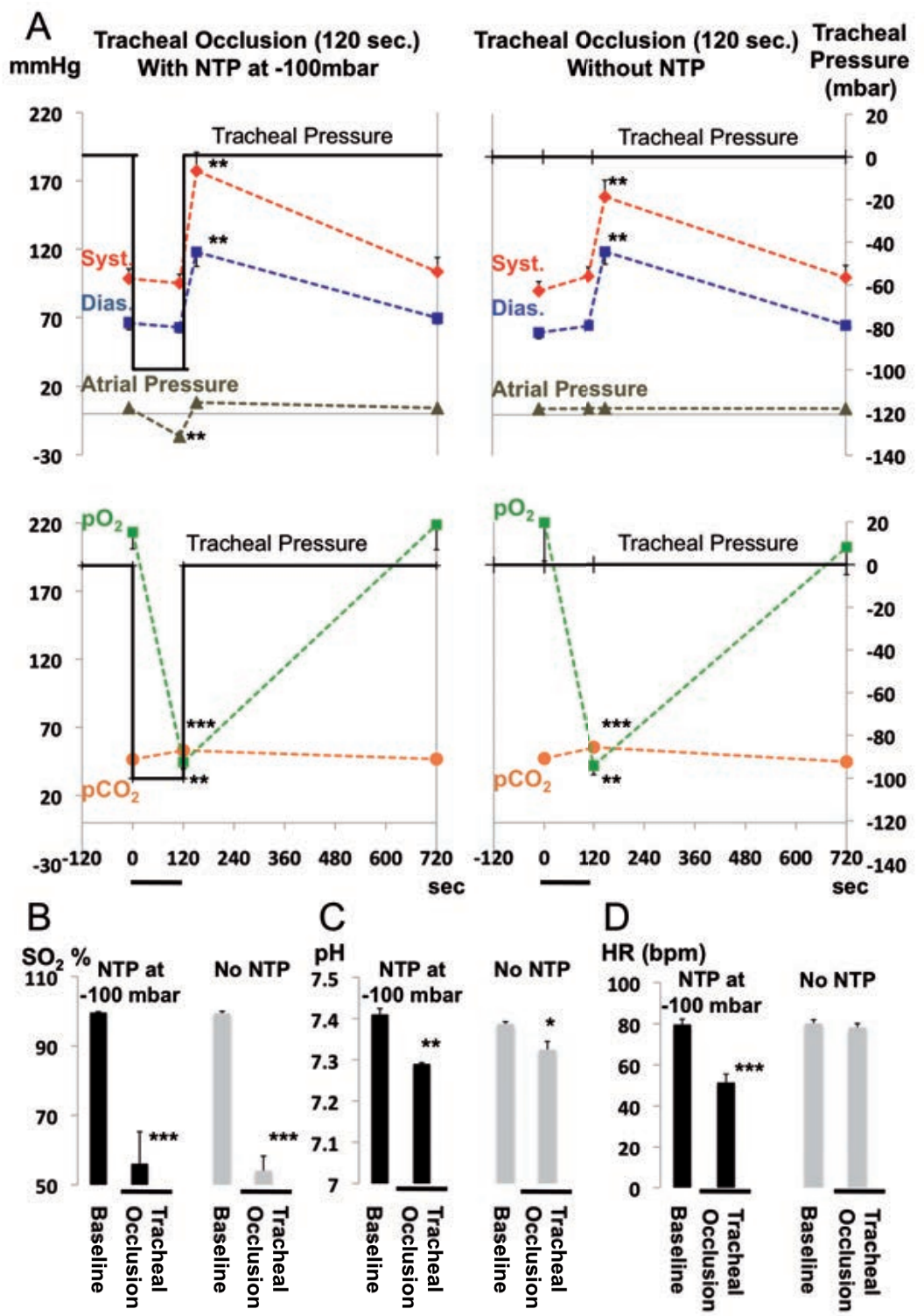

Figure 2: Effect of 2 minutes of tracheal occlusion with (left) and without (right) applied negative tracheal pressure at $\mathbf{- 1 0 0}$ mbar (NTP, continuous line) on hemodynamics and blood gases (dotted line): changes in systolic (Syst.) and diastolic (Dias.) blood pressure and atrial pressure (at the top) and pO2 and pCO2 (at the bottom) are shown (A). Bellow, changes in SO2 (B), pH (C) and heart rate (HR) (D) are shown. $\left({ }^{*} \mathrm{p}<0.01,{ }^{* *} \mathrm{p}<0.001,{ }^{* *} \mathrm{p}<0.0001\right.$ vs. before tracheal occlusion) 


\section{Effect of NTP on AERP, MAP-duration and AF-inducibility}

Figure 3 shows an individual example of the effect of tracheal occlusion with (left) and without (right) applied NTP on AERP (Fig. 3 A) and MAP-duration (Fig. 3 B). Two minutes of tracheal occlusion with -100 mbar NTP significantly shortened the AERP from $161.2 \pm 2.6 \mathrm{~ms}$ to $96.3 \pm 9.0 \mathrm{~ms}(\mathrm{p}<0.0001)$ and shortened the MAP at $70 \%$ of repolarisation from $168.9 \pm 2.1 \mathrm{~ms}$ to $102.2 \pm 7.2 \mathrm{~ms}$ ( $p<0.0001$, representative MAPs are shown in Fig. 3). By contrast, two minutes of tracheal occlusion without applied NTP had no significant effect on AERP (155.5 $\pm 1.8 \mathrm{~ms}$ vs. $160.0 \pm 2.2 \mathrm{~ms}$ before occlusion, n.s.) or MAP-duration. Progressive NTP-induced AERP- and MAPshortening was associated with increased inducibilty of AF by premature stimuli during the AERP-measurement from $0 \%$ during normal breathing or during tracheal occlusion without applied NTP to $91 \%$ during tracheal occlusion with applied NTP $(p<0.00001)$ (Fig. 3 C). Release of tracheal occlusion resulted in a prompt restoration of sinus rhythm within $2.4 \pm 1.1 \mathrm{~min}$ and NTP-induced electrophysiological changes were completely reversible in all animals. AF was not inducible by premature stimuli during normal breathing or tracheal occlusion without applied NTP. Additionally, in 3 control animals (data not shown), 4 repetitive full electrophysiological protocols including tracheal occlusions with and without applied NTP were conducted over a time frame similar to those of the treated animals. NTPinduced changes in electrophysiology and recovery at each repetition over time were constant. 
A

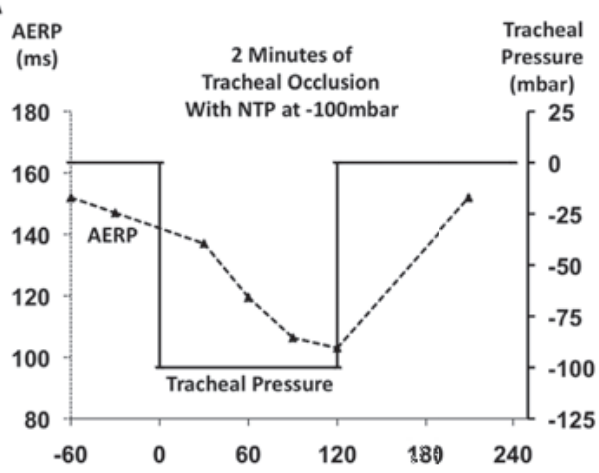

B

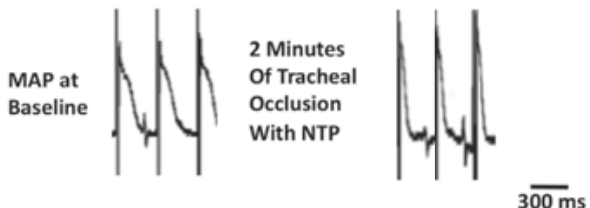

C
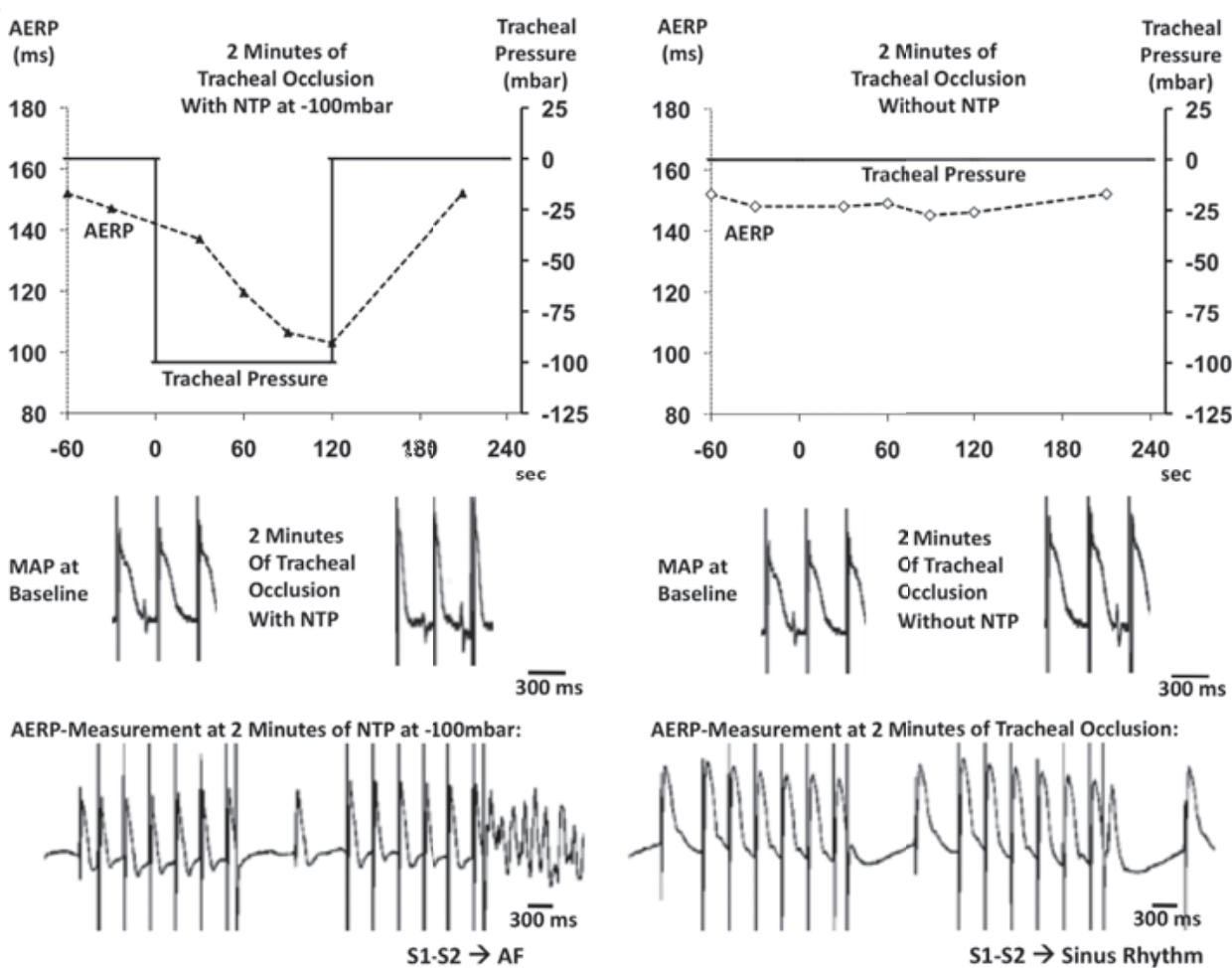

AERP-Measurement at 2 Minutes of Tracheal Occlusion:

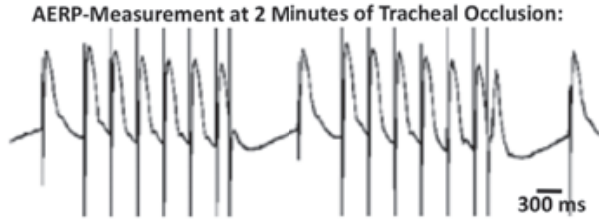

S1-S2 $\rightarrow$ Sinus Rhythm

Figure 3: Changes in atrial electrophysiology during 2 min of tracheal occlusion with (left) or without (right) applied negative tracheal pressure (NTP) at -100 mbar. An individual example of changes in AERP is shown in figure 3 (A) and corresponding original recordings of monophasic action potentials (MAP) at baseline and at 2 minutes of tracheal occlusion are shown in figure 3 (B). In figure 3 (C), an individual example of corresponding MAP signals during representative AERPmeasurements at the end of 2 minutes of tracheal occlusion is shown. Applied NTP at -100 mbar resulted in a progressive shortening of AERP and MAP and AF was inducible by a premature beat during the S1-S2 AERP measurement procedure. 


\section{Effect of vagal blockade, NHE-blockade and $\mathrm{K}_{\mathrm{ATP}}$-channel-blockade}

An individual example of the effect of vagal blockade and $\mathrm{K}_{\mathrm{ATP}}$-channel-blockade on AERP during tracheal occlusion with applied NTP is shown in figure 4. The effect of vagal blockade, $\mathrm{K}_{\mathrm{ATP}}$-channel-blockade and NHE-blockade on NTP-induced AFinducibility and AERP is summarized in figure 5 and table 1.

Table 1: Atrial Effective Refractory Period (AERP, ms) During Tracheal Occlusion With or Without Applied Negative Tracheal Pressure (NTP, -100 mbar). Effect of Vagal Blockade, Glibenclamide and Cariporide.

\begin{tabular}{|c|c|c|c|c|c|c|}
\hline & & $\begin{array}{l}\text { Tracheal Occlusio } \\
\text { - 100mbar } \\
\text { Before Occlusion }\end{array}$ & $\begin{array}{l}\text { on With NTP at } \\
2 \text { min of Occlusion }\end{array}$ & $\begin{array}{l}\text { Tracheal Occlusio } \\
\text { NTP } \\
\text { Before occlusion }\end{array}$ & $\begin{array}{l}\text { on Without Applied } \\
2 \text { min of Occlusion }\end{array}$ & \\
\hline \multicolumn{7}{|l|}{ Group 1} \\
\hline & Baseline & $161.2 \pm 2.6$ & $96.3 \pm 9.0^{*}$ & $160.0 \pm 2.2$ & $155.5 \pm 1.8$ & $n=6$ \\
\hline & + Atropine & $153.7 \pm 6.7$ & $152.0 \pm 6.7++$ & $151.2 \pm 3.5$ & $145.3 \pm 4.6$ & $n=6$ \\
\hline & + Vagotomy & $158.7 \pm 5.8$ & $156.2 \pm 5.71++$ & $148.7 \pm 0.9$ & $150.3 \pm 4.6$ & $n=6$ \\
\hline & + Glibenclamide & $152.9 \pm 5.6$ & $180.6 \pm 5.2+\ddagger$ & $158.7 \pm 3.0$ & $146.2 \pm 6.9$ & $n=6$ \\
\hline \multicolumn{7}{|l|}{ Group 2} \\
\hline & Baseline & $151.4 \pm 4.3$ & $109.8 \pm 6.6^{*}$ & $151.4 \pm 8.6$ & $148.1 \pm 8.6$ & $n=5$ \\
\hline & + Glibenclamide & $142.8 \pm 6.6$ & $103.0 \pm 8.9 \ddagger$ & $146.0 \pm 10.7$ & $142.0 \pm 9.1$ & $n=5$ \\
\hline & + Atropine & $153.7 \pm 6.1$ & $179.4 \pm 7.4+\&$ & $150.2 \pm 8.2$ & $145.4 \pm 6.1$ & $n=5$ \\
\hline \multicolumn{7}{|l|}{ Group 3} \\
\hline & Baseline & $152.1 \pm 2.9$ & $92.1 \pm 2.9 *$ & $153.3 \pm 1.3$ & $153.3 \pm 2.4$ & $n=3$ \\
\hline & + Cariporide & $153.7 \pm 1.7$ & $83.7 \pm 1.7 \ddagger$ & $150.3 \pm 1.7$ & $153.9 \pm 1.9$ & $n=3$ \\
\hline & + Atropine & $145.3 \pm 1.6$ & $143.7 \pm 3.3+$ & & & $n=3$ \\
\hline
\end{tabular}

Values are shown as mean \pm SEM. Negative tracheal pressure, NTP. Atrial effective refractory periode, AERP

$*$, p<0.0001 vs. AERP before tracheal occlusion

,$+ \mathrm{p}<0.01$ vs. AERP-shortening at NTP at baseline

,$+ p<0.001$ vs. AERP-shortening at NTP at baseline

$\ddagger, p<0.01$ vs. AERP after glibenclamide or cariporide

$\&, p<0.01$ vs. AERP after atropine + vagotomy 
Atropine (group 1, n=6) did not change the AERP significantly at normal breathing, but fully prevented the NTP-induced AERP-shortening (Fig. 4 A and B) and MAPshortening at $70 \%$ of repolarisation (at -100 mbar NTP: $161.2 \pm 3.8 \mathrm{~ms}$ vs. $168.9 \pm 2.9$ ms at baseline, n.s.). After atropine, AF was only inducible in $17 \%$ of all tracheal occlusion maneuvers with applied NTP (vs. 91\% during tracheal occlusion with applied NTP before atropine, $p<0.0001$ ) (Fig. 5). Bilateral vagotomy was performed in all atropine treated pigs and showed no significant additional effect on the AERP over atropine alone. Also, AF was not inducible, neither by premature beats (S2) nor by burst pacing.

Glibenclamide was given after (group 1, Fig. 4 A, B and C) and before vagal blockade (group 2; Fig. 4 D, E and F). Glibenclamide did not change the AERP at normal breathing in neither group. NTP-induced AERP-shortening, AF-duration (2.3 $\pm 0.8 \mathrm{~min}$ vs. $2.5 \pm 1.3 \mathrm{~min}$ at baseline, n.s.) and AF-inducibility (Fig.5) were not inhibited by glibenclamide when given before vagal blockade in group 2 . Glibenclamide given after vagal blockade further increased AERP (180.6 $\pm 5.2 \mathrm{~ms}$ vs. $152.9 \pm 5.1 \mathrm{~ms}$ before NTP, $\mathrm{p}<0.01)$ and MAP at $70 \%$ of repolarisation $(176.2 \pm 6.4 \mathrm{~ms}$ vs. $156.2 \pm 3.1 \mathrm{~ms}$ before NTP, $\mathrm{p}<0.05$ ) during tracheal occlusions with applied NTP towards the end of the NTP procedure. A similar increase in AERP was seen during tracheal occlusion with applied NTP, when atropine was given after glibenclamide (179.4 $\pm 7.4 \mathrm{~ms}$ vs. $153.7 \pm 6.1 \mathrm{~ms}$ before NTP, $p<0.01)$ so that the combinations of atropine and glibenclamide independently of the order of administration showed about the same prolongation of the AERP. 
Cariporide (group 3, table 1) did not change the AERP at normal breathing and did not inhibit NTP-induced AERP-shortening, AF-duration (2.5 $\pm 1.8 \mathrm{~min}$ vs. $2.4 \pm 1.0 \mathrm{~min}$ at baseline, n.s.) MAP-shortening at $70 \%$ of repolarisation $(156.2 \pm 2.5 \mathrm{~ms}$ vs. $82.4 \pm 6.2 \mathrm{~ms}$ at $-100 \mathrm{mbar}$ of NTP, $\mathrm{p}<0.0001$ ) or AF-inducibility (Fig. 5). Again, vagal blockade after cariporide completely prevented NTP-induced AERP-shortening and

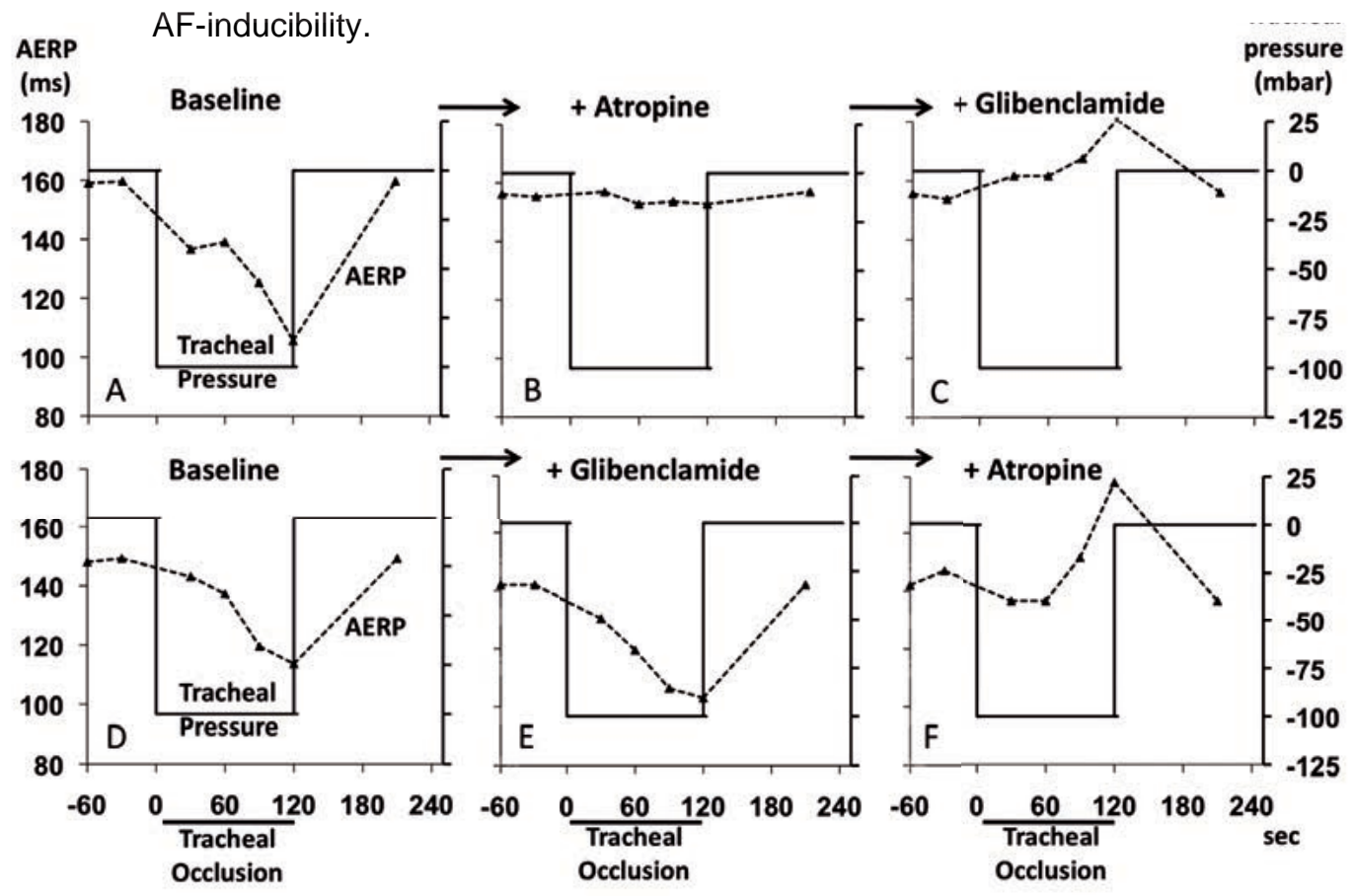

Figure 4: Effect of vagal blockade and glibenclamide on AERP during tracheal occlusion with applied NTP at -100 mbar. Representative time curves of a pig of group 1 (A-C) and a pig of group 2 (D-F) are shown. Effect of NTP (continuous line) on right AERP (dotted line) at baseline and after atropine followed by glibenclamide (group 1) or after glibenclamide followed by atropine (group 2). 


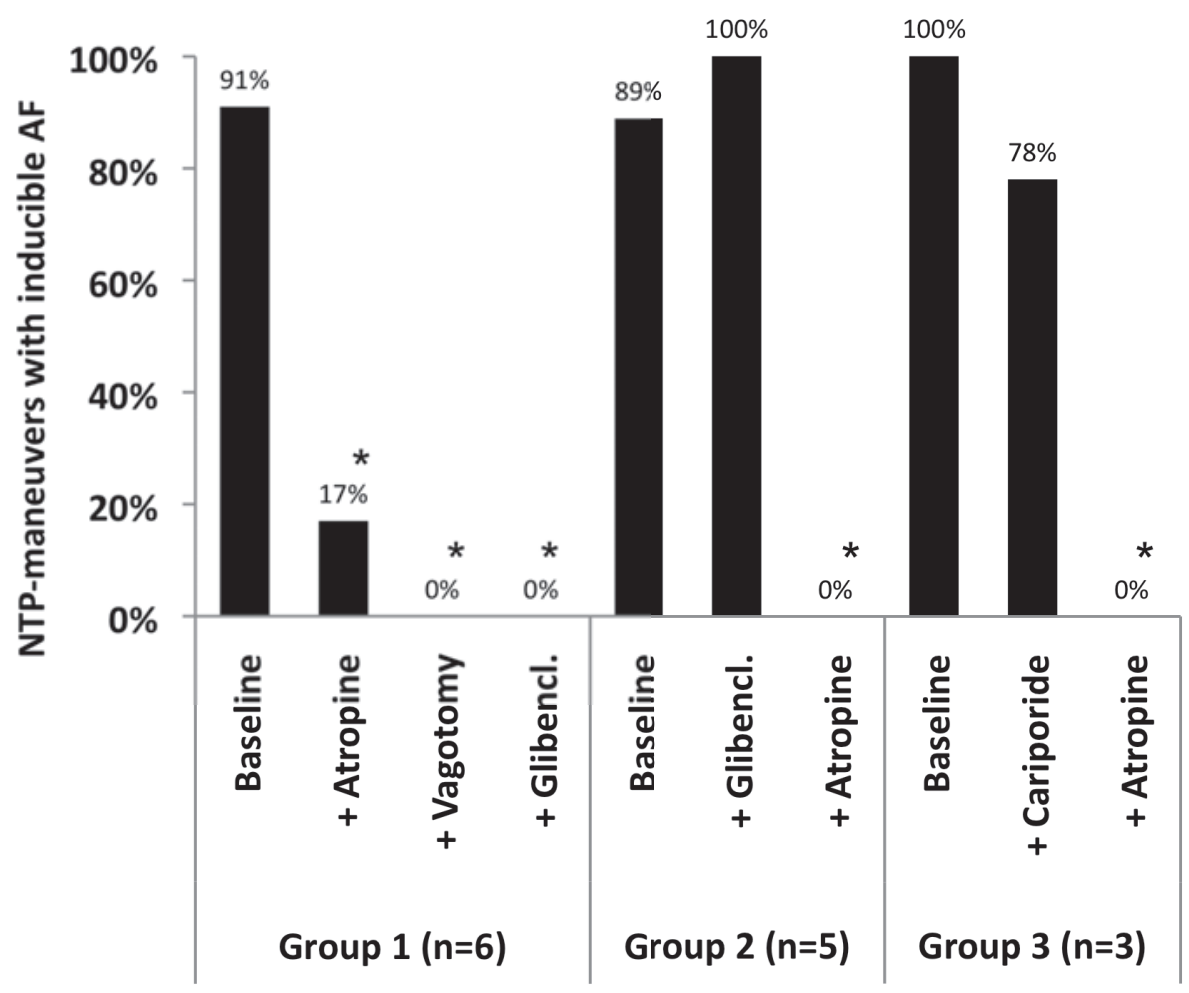

Figure 5: Percent of tracheal occlusions with applied NTP at -100 mbar with inducible AF and effect of vagal blockade, glibenclamide and cariporide. Tracheal occlusion without applied NTP for 2 minutes did not cause AF-inducibility in the pig right atrium. ( ${ }^{*} p<0.001$ vs. respective baseline). 


\section{Discussion}

We report that application of negative tracheal pressure (NTP) during tracheal occlusion but not tracheal occlusion without applied NTP reproducibly and reversibly shortened the AERP and atrial MAP-duration and strongly enhanced inducibility of AF by single premature beats. Electrophysiological changes were mediated by vagal activation since they were completely prevented by atropine or vagotomy. Activation of $\mathrm{Na}^{+} / \mathrm{H}^{+}$-exchanger or $\mathrm{K}_{\mathrm{ATP}}$-channels did not contribute to enhanced AF-inducibility. These findings support a role of NTP during obstructive events as the trigger for OSA-associated AF-susceptibility.

\section{Changes in blood gases, atrial stretch and blood pressure}

Hypoxemia, hypercapnia and acidosis are invariably linked with tracheal occlusion as occurring in OSA ${ }^{9}{ }^{10}$. In our experiments, changes in blood gases appeared during all obstructive maneuvers and did not differ between tracheal occlusion with or without applied NTP. Isolated hypercapnia during modified continuous ventilation without tracheal obstruction and NTP under autonomic blockade resulted in AERPprolongation and reduced AF-vulnerability in a sheep model ${ }^{16}$. In superfused rabbit atria hypoxia prolonged the AERP ${ }^{17}$. In line with these observations, increased AFinducibility due to pronounced AERP- and MAP-shortening only appeared during tracheal occlusion with applied NTP and not during tracheal occlusion without applied NTP but with hypoxia, acidosis and hypercapnia. This indicates that AERPshortening and AF-inducibility is caused by NTP and not by changes in blood gases alone in our model. Possibly two minutes of tracheal occlusion in our model were too short to cause the AERP-prolonging effect observed in previous studies with longer hypoxemic and hypercapnic episodes ${ }^{16}$. Ineffective inspiratory efforts against the occluded upper airway in OSA generate marked increases in negative tracheal 
pressure, resulting in negative intrathoracic pressure. Tracheal occlusion with applied NTP at -100 mbar as observed in OSA patients ${ }^{9,10}$ resulted in a right atrial pressure down to $-16 \mathrm{mmHg}$ suggesting increases in atrial transmural pressure gradients and atrial distension.

Our results show that NTP and not changes in blood gases alone is the mechanism for the observed strong increase in AF-inducibility in our model for OSA. However, AF-episodes were fairly short. Pronounced blood pressure surges due to increased sympathetic activity during the arousal together with changes in blood gases during longterm OSA might lead by a variety of mechanisms to structural atrial remodeling that favors development of persistent $\mathrm{AF}^{3,9,10,18-20}$.

\section{NTP as a potent trigger for vagally induced atrial fibrillation in OSA}

Vagal activation was identified as the major mechanism involved in NTP-induced AERP-shortening. Atropine had no significant effect on AERP at normal breathing or tracheal occlusion without applied NTP, but it prevented NTP-induced AERPshortening in all animals tested. Vagotomy after atropine had no additional effect over atropine alone. After vagotomy, AF was not inducible anymore during NTPmaneuvers, neither by premature beats, nor by burst pacing. Our findings are in line with previous studies showing that the vagal tone is increased during obstructive events in patients with OSA ${ }^{8}$. Interestingly, ablation of ganglionated plexi or combined autonomic blockade inhibited the appearance of apnea induced AF during a 2 min apneic interval in dogs ${ }^{21}$. This dog-model is used as a model for central apnea or Cheyne-Stokes respiration. Our pig model with applied NTP mimics conditions known from obstructive sleep apnea. Our results suggest that the NTP during inspiration against the obstructed airway in OSA is the trigger for vagally induced $\mathrm{AF}$ in OSA while changes in blood gases alone during simple apnea in 
central sleep apnea just play a minor role for acute electrophysiological changes in the atrium. However our studies do not disclose how NTP-maneuvers enhance vagal activity. NTP could stimulate vagal afferent nerves in the lung, the heart and the atria. With respect to atrial afferent vagal activity so-called B-receptors are present in the atria acting as volume receptors ${ }^{8}$. An increase in the transmural pressure gradient during NTP might give an inadequate signal of volume overload. Activation of B-receptors is known to inhibit sympathetic activity and to result in vagal activation.

\section{Hypoxia and/or stretch activated NHE-transport and $\mathrm{K}_{\mathrm{ATP}}$-channels}

In order to study mechanisms beyond vagal activation, we characterized the role of the hypoxia and/or stretch activated $\mathrm{Na}^{+} / \mathrm{H}^{+}$-exchanger and $\mathrm{K}_{\text {ATP-channels in NTP- }}$ induced AERP-shortening. We investigated the effect of cariporide, a blocker of the cardiac $\mathrm{NHE}^{13}$, and of glibenclamide, a blocker of $\mathrm{K}_{\mathrm{ATP}}-$ channels ${ }^{11}$. Blockade of the cardiac NHE subtype 1 by cariporide did not prevent NTP-induced AERP-shortening in our study. Activation of the NHE occurs in ventricular myocardium during hypoxia and ischemia to regulate intracellular $\mathrm{pH}$. It is known to be proarrhythmic possibly by changes in intracellular calcium ${ }^{12}$. NHE is an important mechanism underlying shortterm but not long-term atrial electrophysiological remodeling from rapid atrial rates

and from atrial ischemia ${ }^{22}$. Our data argues against a role for NHE subtype 1 for NTP-induced AERP-shortening. The role of $\mathrm{K}_{\mathrm{ATP}}$-channels seems to be more complex as suggested by the differential effects of glibenclamide, a blocker of $\mathrm{K}_{\text {ATP- }}$ channels. Glibenclamide given after vagal blockade further prolonged the AERP in the course of the NTP procedure indicating activation of $\mathrm{K}_{\text {ATP-channels. }} \mathrm{K}_{\text {ATP- }}$ channels may be opened by hypoxia and opening may be enhanced by increased transmural pressure gradients and stretch in the atria during the NTP-procedure 
since these channels were reported to be stretch-sensitive ${ }^{11}$. By contrast, glibenclamide given before atropine did not prevent NTP-induced AERP-shortening

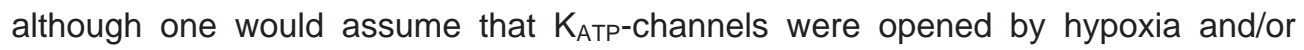
stretch, too. The absence of an obvious effect by glibenclamide when given before atropine is in line with the observation that glibenclamide did not prevent tachypacing-induced AERP-shortening in the atrium ${ }^{23}$. The differential effect of glibenclamide could be explained by previous findings showing that glibenclamide can favor the release of $\mathrm{ACh}$ in the atrium ${ }^{24}$. This means that a facilitation of $\mathrm{ACh}$ release by glibenclamide would result in a more pronounced AERP-shortening which could have neutralized an AERP-prolonging effect by blockade of $\mathrm{K}_{\text {ATP-channels in }}$ atrial cardiomyocytes. Atropine would completely eliminate such a vagal enhancing effect and an AERP-prolonging component of $\mathrm{K}_{\mathrm{ATP}}$-channel-blockade in atrial cardiomyocytes could be unmasked. In line with this assumption, the combination of atropine and glibenclamide resulted in almost the same AERP-prolongation independent of the order of administration. Additionally, tracheal occlusion without applied NTP after atropine plus glibenclamide did not cause AERP-prolongation.

\section{Limitations}

There are some limitations but also strengths of our study. Experimental conditions chosen to induce AERP-shortening were strong with regard to the duration of the challenge and the extent of NTP. Spontaneous breathing of our anesthetized pigs against the occluded trachea developed tracheal pressures of -40 mbar only possibly due to anesthesia, young age, weight and species. We applied NTPs comparable to those reported for subjects with OSA ${ }^{9,10}$. The NTP had to be applied continuously for technical reasons. The tracheal pressure changes caused by the breathing attempts of the animal during tracheal occlusion with applied NTP ranged 
between -110 and -90 mbar. We applied a premature beat to induce AF. While AERP shortening would strongly facilitate reentry, a trigger is still needed to induce AF. In some animals, we observed that AF was induced by spontaneous atrial premature beats, but this spontaneous induction of AF was too rare for a systematic evaluation. A further limitation is that our investigations were made at a single fixed anatomical position in the right atrium although the left atrium may be more relevant for the induction of AF. We did not look at inhomogeneity in conduction, conduction velocity or spatial distribution of refractoriness in the atrium. Investigations in the left atrium and investigations of conduction inhomogeneities would require thoracotomy, which is not possible with urethane anesthesia. Thoracotomy would result in a more artificial situation which would not reflect OSA. Although we tried a lot, our pig-model did not allow to control blood gases content during NTP maneuvers.

\section{Conclusions}

In the present study we found that application of strong NTP during tracheal occlusion shortened AERP and MAP-duration and increased AF-vulnerability in a pig model for OSA. These electrophysiological changes were clearly mediated by vagal activation. By contrast, comparable changes in blood gases during tracheal occlusion without applied NTP did not have such an electrophysiological effect. The results strongly support the hypothesis that NTP during inspiration against the obstructed airway and not changes in blood gases alone is the most relevant mechanism for vagally induced AF-susceptibility in OSA. Additionally, the blockade

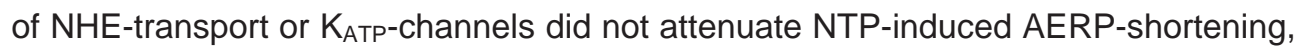

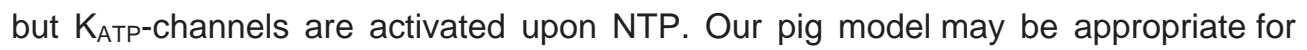
the development of new treatment strategies to treat therapy resistant AF in OSA and can be used as a new model for vagal-AF. 


\section{References}

1. Johnson KG, Johnson DC: Obstructive sleep apnea is a risk factor for stroke and atrial fibrillation. Chest 2010; 138:239.

2. Gami AS, Pressman G, Caples SM, et al.: Association of atrial fibrillation and obstructive sleep apnea. Circulation 2004; 110:364-367.

3. Kasai T, Bradley TD: Obstructive sleep apnea and heart failure: pathophysiologic and therapeutic implications. J Am Coll Cardiol 2011; 57:119-127.

4. Jongnarangsin K, Chugh A, Good E, et al.: Body mass index, obstructive sleep apnea, and outcomes of catheter ablation of atrial fibrillation. $J$ Cardiovasc Electrophysiol 2008; 19:668-672.

5. Matiello $M$, Nadal $M$, Tamborero $D$, et al.: Low efficacy of atrial fibrillation ablation in severe obstructive sleep apnea patients. Europace 2010; 2:10841089.

6. Kanagala R, Murali NS, Friedman PA, et al.: Obstructive sleep apnea and the recurrence of atrial fibrillation. Circulation 2003; 107:2589-2594.

7. Patterson E, Lazzara R, Szabo B, et al.: Sodium-calcium exchange initiated by the $\mathrm{Ca} 2+$ transient: an arrhythmia trigger within pulmonary veins. J Am Coll Cardiol 2006; 47:1196-1206.

8. Somers VK, Dyken ME, Mark AL, Abboud FM: Parasympathetic hyperresponsiveness and bradyarrhythmias during apnea in hypertension. Clin Auton Res 1992; 2:171-176. 
9. Parish JM, Somers VK: Obstructive sleep apnea and cardiovascular disease. Mayo Clin Proc 2004; 79:1036-1046.

10. Kohler M, Stradling JR: Mechanisms of vascular damage in obstructive sleep apnea. Nat Rev Cardiol 2010; 7:677-685.

11. Van Wagoner DR: Mechanosensitive gating of atrial ATP-sensitive potassium channels. Circ Res 1993; 72:973-983.

12. Khandoudi N, Bernard M, Cozzone P, Feuvray D: Intracellular pH and role of $\mathrm{Na}+/ \mathrm{H}+$ exchange during ischaemia and reperfusion of normal and diabetic rat hearts. Cardiovasc Res 1990; 24:873-878.

13. Scholz W, Albus U, Counillon L, et al.: Protective effects of HOE642, a selective sodium-hydrogen exchange subtype 1 inhibitor, on cardiac ischaemia and reperfusion. Cardiovasc Res 1995; 29:260-268.

14. Orban M, Bruce CJ, Pressman GS, et al.: Dynamic changes of left ventricular performance and left atrial volume induced by the mueller maneuver in healthy young adults and implications for obstructive sleep apnea, atrial fibrillation, and heart failure. Am J Cardiol 2008; 102:1557-1561.

15. Wirth KJ, Knobloch K: Differential effects of dofetilide, amiodarone, and class Ic drugs on left and right atrial refractoriness and left atrial vulnerability in pigs. Naunyn Schmiedebergs Arch Pharmacol 2001; 363:166-174.

16. Stevenson $\mathrm{IH}$, Roberts-Thomson KC, Kistler PM, et al.: Atrial electrophysiology is altered by acute hypercapnia but not hypoxemia: Implications for promotion of atrial fibrillation in pulmonary disease and sleep apnea. Heart Rhythm 2010; 7:1271-1272. 
17. Lammers WJ, Kirchhof C, Bonke FI, Allessie MA: Vulnerability of rabbit atrium to reentry by hypoxia. Role of inhomogeneity in conduction and wavelength. Am J Physiol 1992; 262:H47-55.

18. Jais P, Peng JT, Shah DC, et al:: Left ventricular diastolic dysfunction in patients with so-called lone atrial fibrillation. J Cardiovasc Electrophysiol 2000, $11: 623-625$.

19. Choisy SCM, Arberry LA, Hancox JC, James AF: Increased Susceptibility to Atrial Tachyarrhythmia in Spontaneously Hypertensive Rat Hearts. Hypertension 2010; 49:498-505.

20. Li D, Fareh S, Leung TK, Nattel S: Promotion of atrial fibrillation by heart failure in dogs: atrial remodeling of a different sort. Circulation 1999; 100:8795.

21. Ghias M, Scherlag BJ, Lu Z, et al.: The role of ganglionated plexi in apnearelated atrial fibrillation. J Am Coll Cardiol 2009; 54:2075-2083.

22. Jayachandran JV, Zipes DP, Weksler J, Olgin JE: Role of the $\mathrm{Na}(+) / \mathrm{H}(+)$ exchanger in short-term atrial electrophysiological remodeling. Circulation 2000;101:1861-1866.

23. Wijffels MC, Kirchhof CJ, Dorland R, Power J, Allessie MA: Electrical remodeling due to atrial fibrillation in chronically instrumented conscious goats: roles of neurohumoral changes, ischemia, atrial stretch, and high rate of electrical activation. Circulation 1997;96:3710-3720. 
24. Fabiani ME, Story DF: Effects of cromakalim, pinacidil and glibenclamide on cholinergic transmission in rat isolated atria. Pharmacol Res 1995; 32:155163. 


\section{Chapter 4}

Combined Blockade of Early And Late Activated Atrial Potassium Currents Suppresses Atrial Fibrillation in a Pig Model of Obstructive Apnea.

Dominik Linz; Hans-Ruprecht Neuberger; Michael Böhm; Klaus Wirth; Ulrich Schotten.

Heart Rhythm. 2011 Dec;8(12):1933-9. 


\section{Abstract}

Background: Negative tracheal pressure (NTP) during tracheal obstruction in obstructive apnea increases vagal tone and causes pronounced shortening of the atrial effective refractory period (AERP) thereby perpetuating atrial fibrillation (AF). The role of different atrial potassium channels under those conditions has not been investigated._This study aimed to evaluate the atrial effects of blockade of the late activated potassium current $\left(\mathrm{I}_{\mathrm{Kr}}\right)$ by sotalol, of blockade of the early activated potassium currents $\left(\mathrm{I}_{\mathrm{Kur}} / \mathrm{I}_{\mathrm{to}}\right)$ by AVE0118 and of the multichannel blocker amiodarone during tracheal occlusions with applied NTP.

Methods: 21 pigs were anesthetized and an endotracheal tube was placed to apply NTP (up to -100 mbar) comparable to clinically observed obstructive sleep apnea for 2 minutes. Right AERP and AF-inducibility were measured transvenously by a monophasic action potential recording and stimulation catheter.

Results: Tracheal occlusion with applied NTP caused pronounced AERP-shortening. AF was inducible during all NTP-maneuvers. Neither blockade of $\mathrm{I}_{\mathrm{kr}}$ by sotalol, nor blockade of $\mathrm{I}_{\mathrm{Kur}} / \mathrm{I}_{\text {to }}$ by AVE0118 nor amiodarone affected NTP-induced AERPshortening although they prolonged the AERP during normal breathing. Atropine given after amiodarone completely inhibited NTP-induced AERP-shortening. The combined blockade of $\mathrm{I}_{\mathrm{Kr}}$ and $\mathrm{I}_{\mathrm{Kur}} / \mathrm{I}_{\mathrm{to}}$ by sotalol plus AVE0118, however, attenuated NTP-induced AERP-shortening and AF-inducibility independent of the order of administration.

Conclusions: The atrial proarrhythmic effect of NTP simulating obstructive apneas is difficult to inhibit by class III antiarrhythmic drugs. Neither amiodarone nor blockade of $\mathrm{I}_{\mathrm{Kr}}$ or $\mathrm{I}_{\mathrm{Kur}} / \mathrm{I}_{\mathrm{to}}$ attenuated NTP-induced AERP-shortening. However, the combined blockade of $I_{\mathrm{Kur}} / I_{\text {to }}$ and $I_{\mathrm{kr}}$ suppressed NTP-induced AERP-shortening. 


\section{Introduction}

Patients with atrial fibrillation (AF) show a high prevalence of obstructive sleep apnea (OSA), ranging from 32 to $49 \%{ }^{1-3}$. Patients with untreated OSA have a higher recurrence of AF after cardioversion and after pulmonary vein antrum isolation ${ }^{4-7}$. Previously, we described atrial electrophysiological changes in a pig model for obstructive apneas $^{8}$. Increased susceptibility to AF was caused by a pronounced shortening of the atrial effective refractory period (AERP) and monophasic action potentials (MAP) in the pig right atrium as a response to applied negative tracheal pressure (NTP). Negative tracheal pressure occurs in patients with OSA due to diaphragma contraction during upper airway obstruction and results in a negative intrathoracic pressure and increased transmural pressure gradients ${ }^{9-11}$. Vagal activity was identified as the major mechanism involved in NTP-induced AERPshortening in this model, since NTP-induced AERP-shortening, NTP-induced MAPshortening and AF-inducibility were completely prevented by atropine. Acetylcholine dependent potassium channels are discussed to be one of the most relevant components by which vagal tone induces AERP-shortening in the atrium ${ }^{12}$. The aim of this study was to investigate the effect of the blockade of different potassium channels on NTP-induced AERP-shortening, NTP-induced MAP-shortening and AFinducibility during obstructive events. We tested the effect of the blockade of the early activated potassium currents $I_{\text {Kur }}$ (ultrarapid delayed rectifier potassium current) and $I_{\text {to }}$ (transient outward current) by AVE0118 ${ }^{13}$ compared with the blockade of the late activated potassium currents $\mathrm{IKr}$ (the rapid component of delayed rectifier potassium current) by sotalol ${ }^{14}$ on AERP, MAP and AF-inducibility during normal breathing and during tracheal obstruction with applied NTP. Since AERP-shortening induced by NTP is vagally mediated involving acetylcholine sensitive potassium channels $\left(\mathrm{K}_{\mathrm{ACh}}\right)$ 
we tested the antiarrhythmic effects of the multichannel blocker amiodarone, which has been shown to block $\mathrm{K}_{\mathrm{ACh}}$-channels in therapeutical concentrations ${ }^{15}$.

\section{Methods}

All animal studies conform with the Guideline for the Care and Use of Laboratory Animals published by the US National Institutes of Health (NIH Publication No. 85-23, revised 1996).

21 closed chest male castrated pigs $(25-30 \mathrm{~kg})$ of the German Landrace were anesthetized with $20 \%$ urethane $(0.8 \mathrm{ml} / \mathrm{kg}$ i.v. load, $0.4 \mathrm{ml} / \mathrm{kg} / \mathrm{h}$ maintainance) and $4 \%$ alpha-chloralose $(0.4 \mathrm{ml} / \mathrm{kg}$ i.v. load, $0.1 \mathrm{ml} / \mathrm{kg} / \mathrm{h}$ maintainance. The pigs were instrumented as described previously ${ }^{8}$. Briefly, a tracheotomy was performed to place an endotracheal tube. This tube was used for tracheal occlusion and to apply different levels of negative tracheal pressures by a negative pressure device. The Mueller maneuver, forced inspiration against airway obstruction, is used in the clinical setting to simulate conditions, particularly negative thoracic pressure, occurring during obstructive sleep apnea. As a modification of this maneuver, we applied NTPs at $-100 \mathrm{mbar}$.

Blood pressure was monitored by a catheter with a pressure transducer at the tip (Millar PC 350; Millar Instruments, Houston, Texas, USA) in the femoral artery. Blood gas analysis $\left(\mathrm{pO}_{2}, \mathrm{pCO}_{2}, \mathrm{pH}, \mathrm{O}_{2}\right.$-saturation) was performed before and at the end of each NTP-maneuver. Blood gases at baseline and during tracheal occlusion with applied NTP were not influenced by the different drugs (data not shown). The cervical vagi were dissected and bilateral vagotomy was performed in some of the pigs. 


\section{Electrophysiological measurements}

Atrial effective refractory period (AERP) at a basic cycle length of $300 \mathrm{~ms}$ was measured before, during, and after the NTP-maneuvers as described previously ${ }^{16}$. Briefly, atrial responses to the pacing procedure were visualized by monophasic action potentials (MAP) from the endocardium of the right atrium via a MAP pacing catheter (combined MAP- \& stimulation catheter, 7F, Föhr Medical Instruments GMBH, Seeheim, Germany). The catheter was inserted via a femoral vein. The tip of the catheter was advanced to the lateral right atrium to record a stable and sharp MAP-signal. This catheter was left at one location to ensure AERP determination at a fixed anatomic site throughout the experiment. A train of 5 basic stimuli (S1, pulse duration $1 \mathrm{~ms}$ ) at twice diastolic pacing threshold was followed by an extrastimulus (S2) starting about $30 \mathrm{~ms}$ below the expected AERP with a 5-ms increment (UHS 20, universal heart stimulator; Biotronik, Berlin, Germany). The shortest coupling interval able to elicit a propagated atrial response minus $3 \mathrm{~ms}$ was taken as the AERP. The AERP-measurement frequently induced episodes of AF during NTP-maneuvers (AFinducibility). Additionally, inducible AF-duration was determined.

For analysis of right atrial MAP-signals, MAP electrode and position of the electrode were the same as used for AERP measurements (see above). A particular effort had to be made to obtain atrial MAPs of sufficient quality. MAP-signals were just used for analysis, if a regular baseline and amplitude of the MAPs could be maintained for at least $30 \mathrm{sec}$ and if the shape of the MAP was stable during NTP-procedures. Right atrial MAP duration (MAP) was evaluated from $70 \%$ repolarization during regular pacing (BCL=300ms). 


\section{Drugs}

Amiodarone (3mg/kg ("low dose") and $5 \mathrm{mg} / \mathrm{kg}$ ("high dose"), synthetized at SanofiAventis, Frankfurt, Germany), AVE0118 (3mg/kg, synthetized at Sanofi-Aventis, Frankfurt, Germany), atropine $(0.02 \mathrm{mg} / \mathrm{kg})$ and sotalol $(1 \mathrm{mg} / \mathrm{kg}$, d,l-sotalol synthetized by Bristol-Myers Squibb, New York, US) were dissolved in $0.5 \mathrm{ml}$ DMSO and $2.5 \mathrm{ml}$ of polyethyleneglycol (PEG) 400 (Riedel-de Haen, Seelze, Germany). All solutions were injected over 5 minutes. NTP-runs were performed 10 minutes after the end of the infusion. As a control, vehicle as a control was injected 15 min before the second baseline NTP-run in all animals.

\section{Study design and timing sequence}

The study design is shown in figure 1. A complete negative tracheal pressure (NTP) run included atrial effective refractory period (AERP) measurements at baseline and at every 30 sec during a 2 min period of either simple tracheal occlusion at the end of expiration or $2 \mathrm{~min}$ of NTP of -70 or $-100 \mathrm{mbar}$ and at 5 and $15 \mathrm{~min}$ during the recovery period between the different NTP-maneuvers of 15 min. Recovery of the AERP was shown by AERP measurement in the recovery period between the NTPmaneuvers. After the first run, vehicle was given to all animals and a second complete run was performed. After those two complete runs at baseline the different drugs were administered. In group $1(\mathrm{n}=5)$, low dose amiodarone $(3 \mathrm{mg} / \mathrm{kg})$ was followed by atropine. In group $2(n=4)$, high dose amiodarone $(5 \mathrm{mg} / \mathrm{g})$ was followed by bilateral vagotomy. In group 3 ( $n=6)$, sotalol was given first followed by AVE0118. In group $4(\mathrm{n}=6)$, AVE0118 was given first followed by sotalol. Recovery of the AERP and the maintenance of the AERP-prolonging effect of the first drug during normal breathing were shown by an additional AERP measurement in the recovery period between the NTP-maneuvers. 


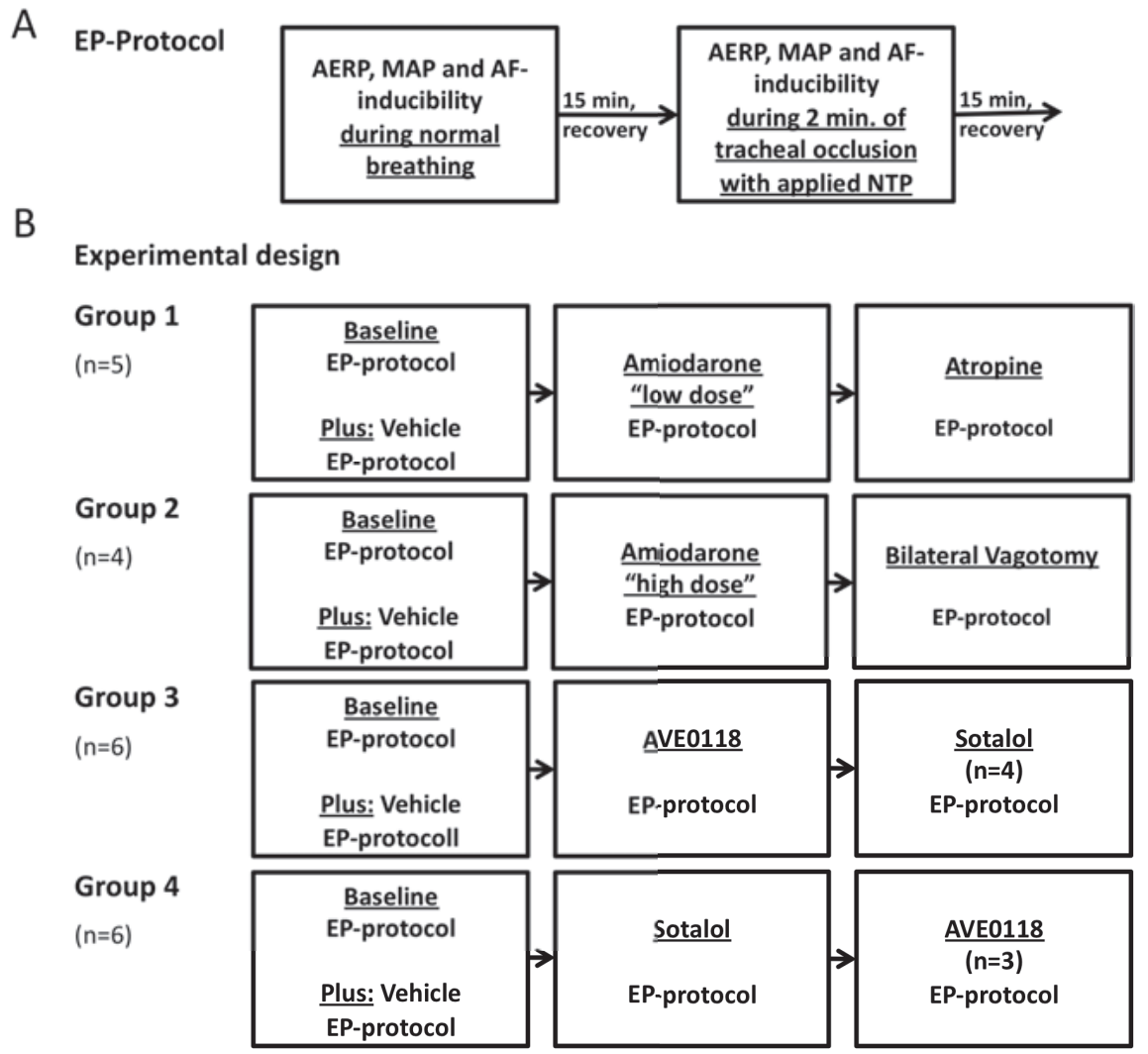

Figure. 1: Flow chart of the electrophysiological protocol (EP-protocol): (A) A complete EP-protocol included AERP-measurement, MAP-recording and AFinducibility during normal breathing and every $30 \mathrm{sec}$ during $2 \mathrm{~min}$ of tracheal occlusion with applied NTP at $-100 \mathrm{mbar}$ with a recovery period of $15 \mathrm{~min}$ between the different NTP maneuvers. (B) The experimental design is shown.

\section{Statistic analysis}

Data are presented as mean \pm SEM. For comparisons of single repeated measures only, a paired Student's t-test was used. For multiple comparisons with the same baseline, repeated-measures ANOVA was used, followed by Dunnet's test to compare individual mean differences if ANOVA was significant. A p value of $<5 \%$ was considered significant. 


\section{Results}

Electrophysiological effects of amiodarone, AVE0118, and sotalol during normal breathing

Figure 2 shows the separate and combined effects of low dose amiodarone and atropine (A), of high dose amiodarone and vagotomy (B) and of AVE0118 and sotalol ( $C$ and $D$ ) on the atrial refractory period during normal breathing. Low dose amiodarone, high dose amiodarone, AVE0118 and sotalol alone increased the AERP by $7.2 \pm 3.2 \mathrm{~ms}, 11.1 \pm 2.1 \mathrm{~ms}, 10.8 \pm 1.7 \mathrm{~ms}$ and $25.8 \pm 4.2 \mathrm{~ms}$, respectively. The AERPprolonging effect of amiodarone, AVE0118 and sotalol during normal breathing at baseline and at the end of recovery period was unchanged within 30 minutes. The combination of AVE0118 and sotalol prolonged the AERP by $45.8 \pm 3.3 \mathrm{~ms}(p<0.01$ vs. the sum of separate AERP-prolongation by sotalol and AVE0118) indicating that the class III effects of AVE0118 and sotalol are synergistic in the pig atrium. AVE0118 (3 mg/kg) did not prolong the QTc interval, (QTc was $413 \pm 15 \mathrm{~ms}$ before and $403 \pm 02 \mathrm{~ms} 10 \mathrm{~min}$ after injection). Sotalol $(1 \mathrm{mg} / \mathrm{kg})$ showed the expected prolongation of the QTc-interval ( $25.1 \%$ increase; from $426 \pm 5 \mathrm{~ms}$ to $533 \pm 12 \mathrm{~ms}$; $p<0.001)$, amiodarone did not induce a significant increase in QTc $(411 \pm 8$ ms before and $420 \pm 5$, n.s.) in pigs. 

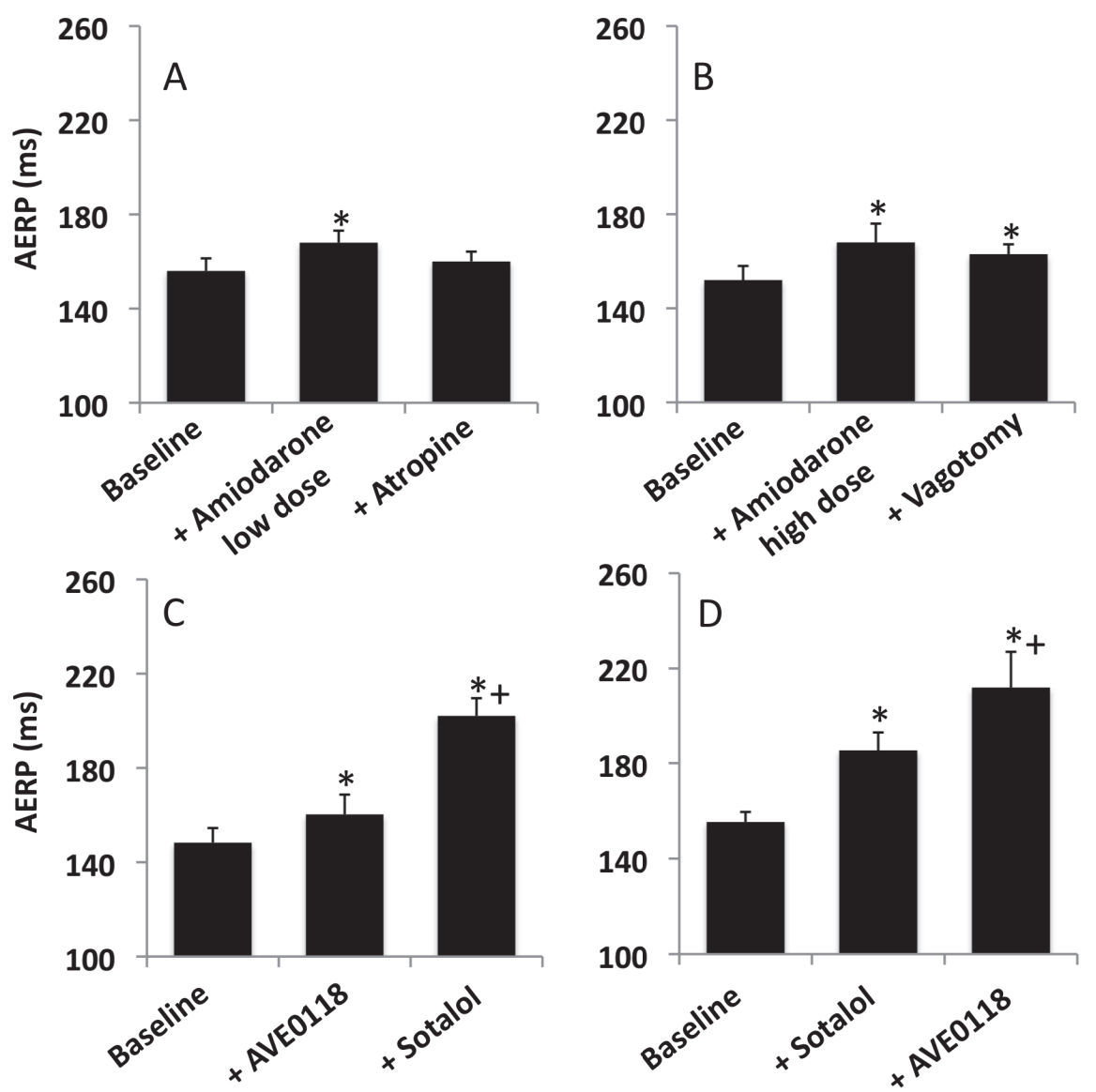

Figure 2: Effect of low dose amiodarone followed by atropine (A), high dose amiodarone followed by bilateral vagotomy (B), AVE0118 followed by sotalol (C) and sotalol followed by AVE0118 (D) on AERP during normal breathing $\left({ }^{*} p<0.01\right.$ vs. respective baseline, $+p<0.01$ vs. after $\operatorname{AVE} 0118(C)$, and vs. after sotalol (D)). 
Effect of amiodarone, AVE0118 and sotalol on NTP-induced AERP- and MAPshortening

Tracheal occlusion without applied NTP did not change the AERP, neither before nor after drug administration. In contrast, the application of NTP induced a pronounced AERP-shortening (from $147.8 \pm 7.9 \mathrm{~ms}$ to $105.1 \pm 10.6 \mathrm{~ms}, \mathrm{p}<0.001$ ) and MAPshortening at $70 \%$ of repolarization (from $145.2 \pm 3.8 \mathrm{~ms}$ vs. $101.9 \pm 2.9 \mathrm{~ms}, \mathrm{p}<0.001$ ). In 3 control animals, 4 repetitive full electrophysiological protocols including NTPmaneuvers were conducted over a time frame similar to those of the treated animals. NTP-induced changes in electrophysiology and recovery at each repetition over time were constant ${ }^{8}$.

In figure 3, the effect of different class III-AADs and their combination on NTPinduced AERP-shortening is shown. Neither low dose nor high dose amiodarone attenuated NTP-induced AERP-shortening. However, both, atropine and bilateral vagotomy applied after the administration of amiodarone completely inhibited NTPinduced AERP-shortening $(155.0 \pm 4.2 \mathrm{~ms}$ to $152.0 \pm 9.8 \mathrm{~ms}$ after atropine vs. $168.1 \pm 5.1 \mathrm{~ms}$ to $94.6 \pm 10.5 \mathrm{~ms}$ after low dose amiodarone, $p<0.01)$ and NTP-induced MAP-shortening at $70 \%$ of repolarization $(159.2 \pm 3.2 \mathrm{~ms}$ to $152.3 \pm 4.9 \mathrm{~ms}$ after atropine vs. $165.3 \pm 2.7 \mathrm{~ms}$ to $97.6 \pm 6.9 \mathrm{~ms}$ after low dose amiodarone, $p<0.01)$. The

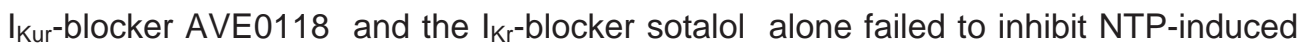
AERP-shortening ( $160.2 \pm 9.5 \mathrm{~ms}$ to $112.2 \pm 9.1 \mathrm{~ms}$ vs. $147.8 \pm 7.9 \mathrm{~ms}$ to $105.1 \pm 10.6 \mathrm{~ms}$ before AVE0118 (n.s.) and $185.3 \pm 7.8 \mathrm{~ms}$ to $131.2 \pm 19.3 \mathrm{~ms}$ vs. $155.3 \pm 4.2 \mathrm{~ms}$ to 92.8 $\pm 7.0 \mathrm{~ms}$ before sotalol (n.s.), respectively) and NTP-induced MAP-shortening (166.6 $\pm 7.5 \mathrm{~ms}$ to $107.1 \pm 8.9 \mathrm{~ms}$ vs. $146.7 \pm 6.5 \mathrm{~ms}$ to $98.5 \pm 9.8 \mathrm{~ms}$ before AVE0118 (n.s.) and $182.5 \pm 6.5 \mathrm{~ms}$ to $127.1 \pm 17.6 \mathrm{~ms}$ vs. $152.2 \pm 3.7 \mathrm{~ms}$ to $97.7 \pm 6.0 \mathrm{~ms}$ before sotalol (n.s.), respectively). In contrast the combination of AVE0118 and sotalol 
significantly reduced NTP-induced AERP-shortening (204.5 $\pm 5.3 \mathrm{~ms}$ to $174.5 \pm 8.3 \mathrm{~ms}$ after the combination of sotalol and AVE0118, $p<0.001$ vs. respective NTP-induced AERP-shortening at baseline before drug administration) and NTP-induced MAPshortening (199.8 $\pm 6.3 \mathrm{~ms}$ to $180.4 \pm 6.9 \mathrm{~ms}$ after the combination of sotalol and AVE0118, $p<0.001$ vs. respective NTP-induced MAP-shortening at baseline before drug administration) independent of the order of administration.

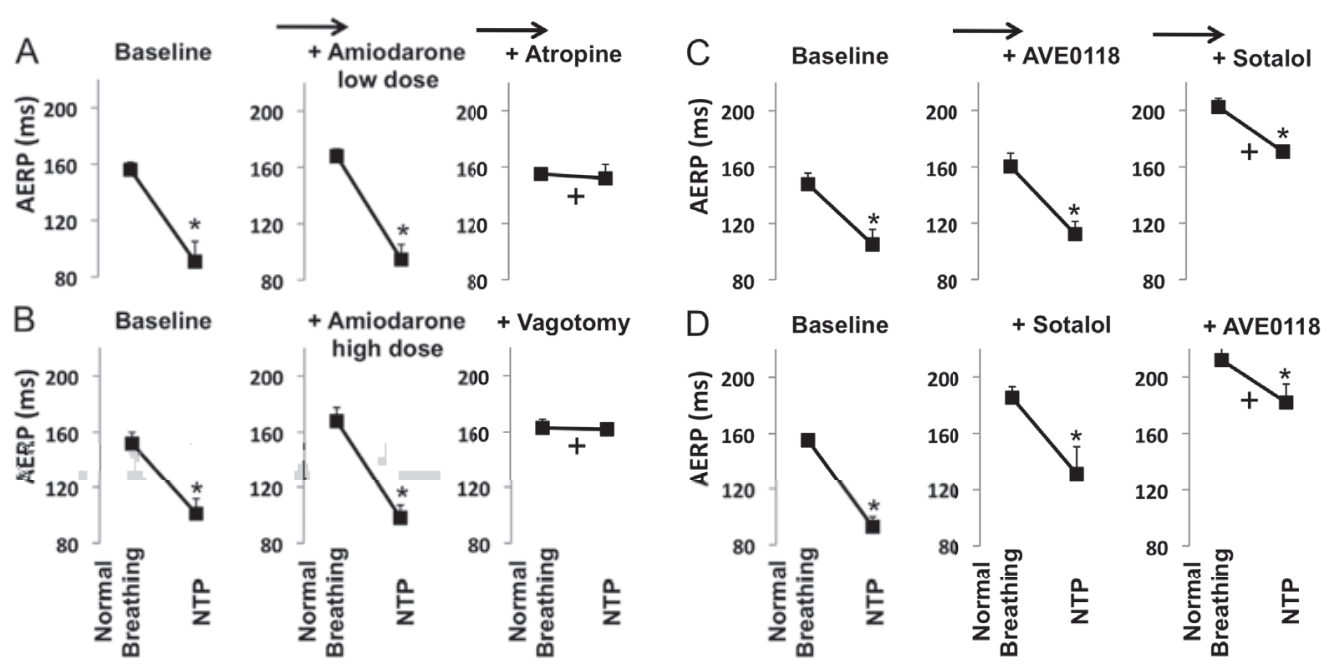

Figure 3: Effect of low dose amiodarone followed by atropine (A), high dose amiodarone followed by bilateral vagotomy (B), AVE0118 followed by sotalol (C) and sotalol followed by AVE0118 (D) on AERP during tracheal occlusion with applied NTP at -100 mbar ( ${ }^{*} p<0.001$ vs. respective baseline, $+p<0.01$ vs. NTP-induced AERP-shortening before drug administration). 
Figure 4 shows changes in AERP (left) during tracheal occlusion without (A) and with (B) applied NTP and MAP-recordings (right) during the respective AERPmeasurements. The combination of sotalol and AVE0118 (C) suppressed NTPinduced AERP-shortening and reduced AF-inducibility.

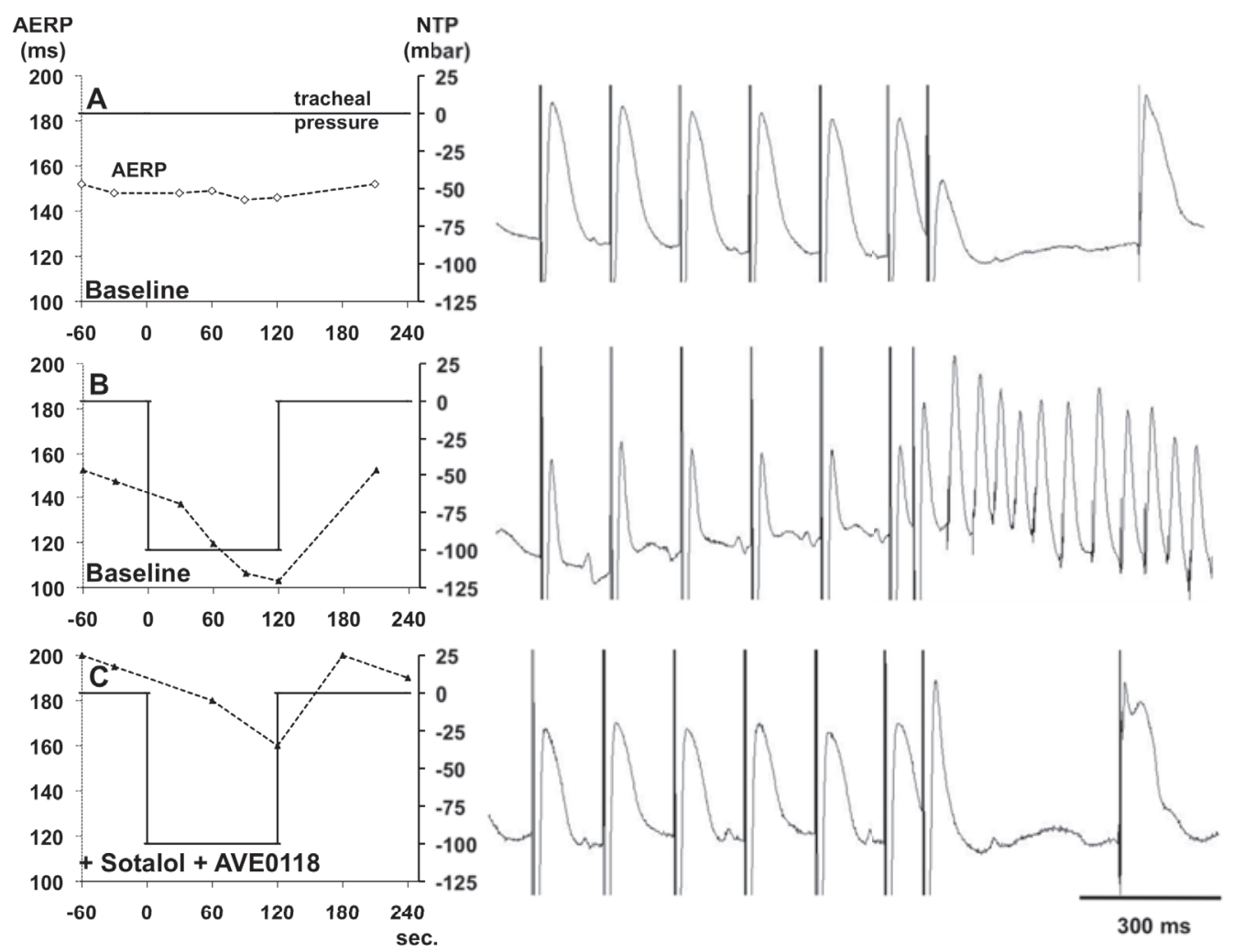

Figure 4: Changes in atrial electrophysiology during 2 min of tracheal occlusion without (A) or with applied negative tracheal pressure (NTP) at -100 mbar before (B) and after sotalol plus AVE0118 (C). A respective individual example of changes in AERP is shown (left) and corresponding MAP signals during representative AERPmeasurements at the end of 2 minutes of tracheal occlusion is shown (right). 
Effect of amiodarone, AVE0118 and sotalol on NTP-Induced AF-Inducibility and AF-duration

AF was not inducible by a single premature stimulus during normal breathing. NTPinduced AERP-shortening was associated with increased inducibilty of AF by a single premature stimulus during the AERP-measurement from $0 \%$ during normal breathing to $87.7 \%$ during tracheal occlusion with applied NTP $(p<0.00001)$. In figure 5 , the effect of different class III-AADs and their combination on NTP-induced AFsusceptibility is shown. Neither low dose nor high dose amiodarone suppressed NTP-induced AF-inducibility significantly. Atropine applied after low dose amiodarone administration and bilateral vagotomy performed after high dose amiodarone completely inhibited NTP-induced AF-inducibility. AVE0118 and sotalol alone did not reduce NTP-induced AF-inducibility. In contrast the combination of sotalol and AVE0118 significantly reduced AF-inducibility during NTP-maneuvers (15\% vs. $87.7 \%$ at baseline before drug administration, $p<0.001$ ).

Inducible AF-duration was just mildly reduced by amiodarone $(89 \pm 8 \mathrm{~s}$ vs. $122 \pm 11 \mathrm{~s}$ at baseline, n.s.), solalol ( $75 \pm 10 \mathrm{~s}$ vs. $119 \pm 10 \mathrm{~s}$ at baseline, $\mathrm{p}<0.05)$ and AVE0118 ( $59 \pm 8$ vs. $130 \pm 12 s$ at baseline, $p<0.01)$. However, the combination of sotalol plus AVE0118 (15 $\pm 8 \mathrm{~s}$ vs. $125 \pm 11 \mathrm{~s}$ at baseline, $\mathrm{p}<0.01)$ caused a pronounced reduction in inducible AF-duration. 


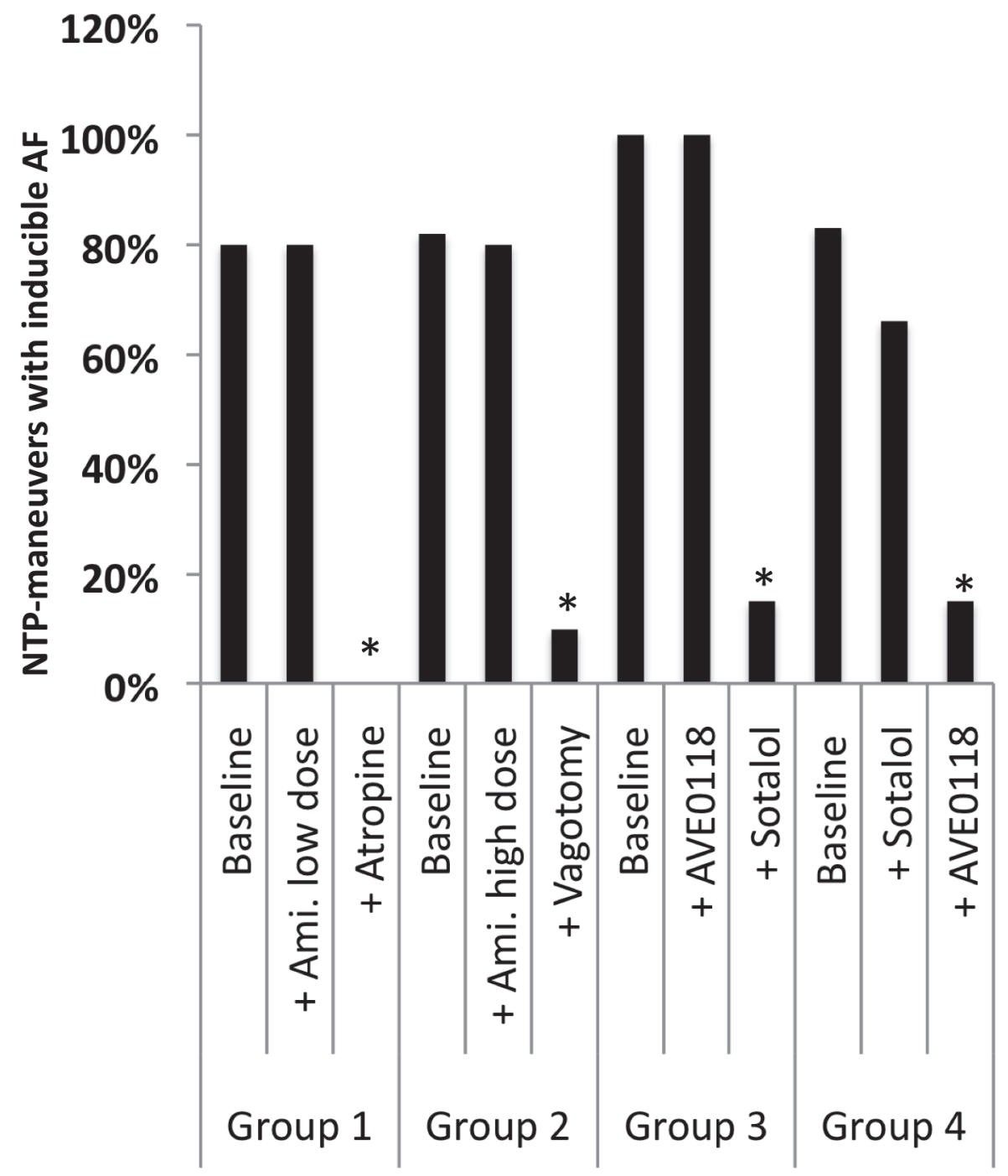

Figure 5: Percent of tracheal occlusions with applied NTP at -100 mbar with inducible AF and effect of low dose amiodarone followed by atropine (group 1), high dose amiodarone followed by bilateral vagotomy (group 2), AVE0118 followed by sotalol (group 3) and sotalol followed by AVE0118 (group 4). Tracheal occlusion without applied NTP for 2 minutes did not cause AF-inducibility in the pig right atrium. ( ${ }^{*} p<0.001$ vs. respective baseline) 


\section{Discussion}

In a pig model simulating the effect of obstructive apneas on the atrium we showed that the combined blockade of the early activated potassium currents $I_{\text {Kur }} / I_{\text {to }}$ (AVE0118) and the late activated potassium current $\mathrm{I}_{\mathrm{Kr}}$ (sotalol) could attenuate NTPinduced AERP-shortening, NTP-induced MAP-shortening and AF-inducibility. By contrast, the $\mathrm{I}_{\mathrm{Kur}} \mathrm{I}_{\mathrm{t} \mathrm{t}^{-}}$or the $\mathrm{I}_{\mathrm{kr}}$-blocker alone was not effective. Surprisingly, amiodarone, which blocks multiple potassium currents including the acetylcholine dependent potassium current $\mathrm{I}_{\mathrm{KACh}}$, did not inhibit NTP-induced AERP-shortening or MAP-shortening although increased vagal tone was clearly identified to mediate electrophysiological changes.

\section{Pig model with applied negative tracheal pressure (NTP) simulating obstructive apneas}

Previously we have shown that AF-inducibility in the right atrium was highly increased by application of NTP in a pig-model simulating obstructive apneas ${ }^{8}$. NTP maneuvers induced pronounced AERP-shortening, MAP-shortening and AFinducibility. Release of NTP resulted in a prompt cardioversion and AERP-shortening and MAP-shortening were reversible. In contrast, hypoxia and hypercapnia alone during tracheal occlusion without applied NTP did not induce electrophysiological changes in the atrium. Increased vagal tone was identified to mediate electrophysiological changes in this model, as suggested by the fact, that AERPshortening, MAP-shortening and enhancement of AF-inducibity were completely prevented by atropine. Acetylcholine stimulates muscarinic M-receptors and activates the $I_{\text {KACh }}$ current in the atria, which is normally small or absent. Increased activity of inward $\mathrm{I}_{\mathrm{KACh}}$ causes shortening of the atrial action potential and thus promotes $\mathrm{AF}^{15}$. 
It has been reported that some class III antiarrhythmic drugs inhibit I IACh-currents. Two mechanisms by which these antiarrhythmic drugs inhibit $I_{\text {KACh }}$ have been proposed: drugs like sotalol ${ }^{18}$ block the muscarinic receptors and others like amiodarone ${ }^{19,20}$ inhibit $I_{\text {KACh }}$ by depressing the function of the $I_{\text {KACh }}$ channel itself. Studies demonstrated that amiodarone effectively suppressed the shortening of atrial effective refractory period and the wavelength for reentry during vagal stimulation, thereby terminating and preventing cholinergic atrial fibrillation ${ }^{15}$. Both sotalol and amiodarone are multichannel blocking antiarrhythmic drugs. Blockade of the $\mathrm{I}_{\mathrm{KACh}}{ }^{-}$ current is considered to be responsible for the superior efficacy of amiodarone compared to other more selective $\mathrm{I}_{\mathrm{kr}}$-blockers like e.g. dofetilide. Additionally, also AVE0118 blocks the $\mathrm{I}_{\mathrm{KACh}}$ at higher dosages ${ }^{13}$. Amiodarone, sotalol and AVE0118 did not sufficiently inhibit NTP-induced AERP-shortening or MAP-shortening. However, atropine administration and bilateral vagotomy after amiodarone were effective in inhibiting AERP-shortening indicating that AERP-shortening was still mediated by vagal activation. The major component of $I_{\text {KACh }}$ arises from the muscarinergic receptor (mAChR) activation. Recent evidence suggests that other mAChR subtypes are present in the heart and that these couple to distinct potassium currents. ${ }^{21-23}$ Whether amiodarone blocks all of those channels is unknown. Possibly, amiodarone does not block $I_{\text {KaCh }}$ channels and sotalol does not block the muscarinic receptors by $100 \%$ in the concentrations used in our study. Incomplete $\mathrm{I}_{\mathrm{KACh}}$-blockade or muscarinic receptor blockade during pronounced increase in AChrelease during NTP-maneuvers might fail to attenuate the AERP-shortening. We tested the effect of a lower and a higher dosage of amiodarone ${ }^{15}$. AERP was prolonged by both dosages of amiodarone during normal breathing, but AERPshortening could not be prevented. In pilot experiments, higher dosages of amiodarone (up to $10 \mathrm{mg} / \mathrm{kg}$ ) failed to inhibit NTP-induced AERP-shortening, caused 
prolongation of QRS-duration possibly due to an increased sodium-channel blocking effect and resulted in relevant hemodynamic changes (data not shown). Therefore we did not systematically investigate higher dosages of amiodarone in our study.

Additionally it is important to consider the duration of increased vagal tone in our model. The atria of our pigs were exposed to an increased vagal tone for only two minutes. In previous studies, vagal tone was increased by a long term infusion of carbachol or acetylcholine or by chronic stimulation of vagal nerve. Long term vagal stimulation might create a substrate which differs significantly from the acute vagal stimulation by NTP. Another explanation may be the stretch sensitivity of $\mathrm{K}_{\mathrm{ACh}}{ }^{-}$ channels. Increased transmural pressure gradients during NTP-maneuvers result in atrial stretch. Atrial stretch has been shown to modulate $I_{\mathrm{KACh}}$-channel activity probably via a direct mechanical effect on the channel protein ${ }^{24}$. This modulation of channel protein structure might influence the effective binding of amiodarone.

\section{$\mathrm{I}_{\mathrm{Kur}} / \mathrm{I}_{\mathrm{to}}$-blocker and $\mathrm{I}_{\mathrm{Kr}}$-blocker}

During normal breathing, the AERP-prolonging effect of $\mathrm{I}_{\mathrm{Kur}} / \mathrm{I}_{\mathrm{to}}$-blockers was lower compared to $\left.\right|_{\mathrm{kr}}$-blockers. This is in line with previous findings that the AERPprolonging effects of $\mathrm{I}_{\mathrm{Kur}}$-blockers are lower in the right pig atrium compared to the left pig atrium. The opposite effect profile applies to $\mathrm{I}_{\mathrm{kr}}$-blockers ${ }^{17}$.

The most obvious atrial electrophysiological change during NTP-maneuvers was the AERP-shortening and MAP-shortening caused by a pronounced vagal activation and thereby an enhanced ACh-dependent potassium current ${ }^{8}$. The strong action potential shortening may depress the plateau of the action potential ${ }^{12}$. The relevance of different potassium currents for the repolarization of the action potential is directly dependent on the AP-duration. During shorter atrial action potentials, the relative importance of the early activating potassium currents such as the $I_{\text {to }}$ and the $I_{\text {Kur }}$ for 
repolarization is increased and the late activated potassium currents such as $I_{\mathrm{Kr}}$ are relegated from an active participation to a passive role (repolarization reserve) ${ }^{25}$. In AF-induced electrical remodeling, the efficacy of $\mathrm{I}_{\mathrm{kr}}$-blockade by sotalol is diminished 26,27 and blockade of I Kur by AVE0118 exerted an enhanced class III effect ${ }^{28-30}$. However, NTP-induced AERP-shortening differs from AF-induced AERP-shortening (electrical remodeling). In AF-induced electrical remodeling, vagal activation seems to play a minor role, as atropine failed to suppress the atrial tachypacing induced AERP-shortening in the goat ${ }^{31}$. During NTP-induced AERP-shorting caused by an increased vagal activation not only the late activated potassium channels $\mathrm{I}_{\mathrm{kr}}$ but also the early activated potassium currents $I_{\text {to }}$ and $I_{\text {Kur }}$ may lose relevance for repolarization due to a strong activation of the $I_{\text {KACh. }}$. Indeed, the blockade of $I_{\text {Kur }} / I_{\text {to }}$ by AVE0118 failed to attenuate NTP induced AERP shortening in our study. This finding is in line with a study by Pandit et al. who demonstrated in vagal-AF in pigs and in a mathematic model that $I_{K u r}$ is an ineffective anti-arrhythmic drug target in vagal AF. ${ }^{32}$ Interestingly, in our experiments the combination of a blocker of the early activated potassium currents $I_{\text {Kur }} / I_{\text {to }}$ (AVE0118) plus a blocker of the late activated potassium current $\mathrm{I}_{\mathrm{Kr}}$ (sotalol) significantly reduced NTP-induced AERP-shortening and NTPinduced MAP-shortening independent of the order of administration. Consequently the premature beats within the AERP-measurements only induced very short episodes of atrial tachycardia. During NTP-induced AERP-shortening caused by a strong activation of $I_{\text {KACh }}$, the $I_{K u r} I_{\text {to }}$ and $I_{\text {KACh }}$ blocker AVE0118 might prolong the early repolarisation and thereby the plateau of the action potential. This restores a condition of repolarisation under which the late activated potassium current $\mathrm{I}_{\mathrm{Kr}}$ regains relevance for repolarisation. A synergistic effect of $I_{K u r}$ and $I_{K r}$-blockers has already been shown in electrical remodelled goat atria ${ }^{29}$ where the combination of AVE0118 and sotalol showed a larger effect than the sum of their separate effects. 
Besides its pronounced class III effect, sotalol also shows some beta-adrenergic blocking effect. However, atenolol and propranolol failed to inhibit NTP-induced AERP-shortening in pilot experiments indicating that sympathetic activation does not seem to play a relevant role in this pig model simulating obstructive events.

\section{Limitations}

We applied a premature beat to induce AF. In some animals, we observed that AF was induced by spontaneous atrial premature beats, but this spontaneous induction of $\mathrm{AF}$ was too rare for a systematic evaluation. A further limitation is that our investigations were made at a single fixed anatomical position in the right atrium. AERP-measurement in the left atrium and the investigation of inhomogeneity in conduction, conduction velocity or spatial distribution of refractoriness in the atrium would have required thoracotomy. In pigs with thoracotomy application of NTP would not be possible. Additionally, we just investigated the acute effect of amiodarone in our model. The chronic administration of amiodarone might have shown a different effect. We tested the dosages as described elsewhere ${ }^{13,16,17}$. Due to limitations in solubility of AVE0118 and pronounced QT-prolongation by sotalol we did not further increase the doses tested in this study.

\section{Conclusion}

In a pig model of obstructive apneas we showed that the combination of a blocker of the early activated potassium current $I_{\text {Kur }} I_{\text {to }}($ AVE0118) and a blocker of the late activated potassium current $\mathrm{I}_{\mathrm{Kr}}$ (sotalol) could reduce NTP-induced AERP-shortening and MAP-shortening, AF-inducibility and AF-duration. By contrast, the $\mathrm{I}_{\mathrm{Kr}^{-}}$or $\mathrm{I}_{\mathrm{Kur}} / \mathrm{I}_{\mathrm{t}^{-}}$ blocker alone was not effective. Surprisingly, amiodarone, a known blocker of the 
acetylcholine dependent potassium channel did not prevent NTP-induced AERPshortening, although increased vagal tone was clearly identified to mediate electrophysiological changes in this model. Our findings suggest that the atrial proarrhythmic effects of obstructive apneas are difficult to inhibit by current antiarrhythmic drugs and that the combined blockade of the early $\left(\mathrm{I}_{\mathrm{Kur}} / \mathrm{I}_{\mathrm{to}}\right)$ and the late activated potassium current $\left(\mathrm{I}_{\mathrm{Kr}}\right)$ may be a promising strategy to treat $\mathrm{AF}$ in patients with OSA. Our data could at least partly explain how AF patients with OSA could become refractory to current class III antiarrhythmic drugs and may therefore be susceptible for progression to permanent AF. 


\section{References}

1. Gami AS, Pressman G, Caples SM, et al.: Association of atrial fibrillation and obstructive sleep apnea. Circulation 2004; 110:364-367.

2. Gami AS, Hodge DO, Herges RM, et al.: Obstructive sleep apnea, obesity, and the risk of incident atrial fibrillation. J Am Coll Cardiol 2007; 49:565-571.

3. Gami AS, Somers VK: Implications of obstructive sleep apnea for atrial fibrillation and sudden cardiac death. J Cardiovasc Electrophysiol 2008; 19:997-1003.

4. Patel D, Mohanty P, Di Biase L, et al.: Safety and efficacy of pulmonary vein antral isolation in patients with obstructive sleep apnea: the impact of continuous positive airway pressure. Circ Arrhythm Electrophysiol 2010; 3:445-451.

5. Jongnarangsin $\mathrm{K}$, Chugh $\mathrm{A}$, Good $\mathrm{E}$, et al.: Body mass index, obstructive sleep apnea, and outcomes of catheter ablation of atrial fibrillation. $J$ Cardiovasc Electrophysiol 2008; 19:668-672.

6. Matiello $M$, Nadal $M$, Tamborero $D$, at al.: Low efficacy of atrial fibrillation ablation in severe obstructive sleep apnoea patients. Europace 2010; 12:1084-1089.

7. Kanagala R, Murali NS, Friedman PA, et al.: Obstructive sleep apnea and the recurrence of atrial fibrillation. Circulation 2003; 107:2589-2594.

8. Linz D, Schotten U, Neuberger HR, Böhm M, Wirth K: Negative Tracheal Pressure During Obstructive Respiratory Events Promotes Atrial Fibrillation by Vagal Activation. Heart Rhythm 2011; Epub ahead of print. 
9. Kasai T, Bradley TD: Obstructive sleep apnea and heart failure: pathophysiologic and therapeutic implications. J Am Coll Cardiol 2011; $57: 119-127$.

10. Parish JM, Somers VK: Obstructive sleep apnea and cardiovascular disease. Mayo Clin Proc 2004; 79:1036-1046.

11. Kohler M, Stradling JR: Mechanisms of vascular damage in obstructive sleep apnea. Nat Rev Cardiol 2010; 7:677-685.

12. Schotten U, Verheule S, Kirchhof P, Goette A: Pathophysiological mechanisms of atrial fibrillation: a translational appraisal. Physiol Rev 2011; $91: 265-325$.

13. Gogelein $\mathrm{H}$, Brendel J, Steinmeyer K, et al.: Effects of the atrial antiarrhythmic drug AVE0118 on cardiac ion channels. Naunyn Schmiedebergs Arch Pharmacol: 2004; 370:183-192.

14. Nattel S: Antiarrhythmic drug classifications. A critical appraisal of their history, present status, and clinical relevance. Drugs 1991; 41:672-701.

15. Gautier P, Guillemare E, Djandjighian L, et al.: In vivo and in vitro characterization of the novel antiarrhythmic agent SSR149744C: electrophysiological, anti-adrenergic, and anti-angiotensin II effects. J Cardiovasc Pharmacol 2004; 44:244-257.

16. Wirth KJ, Knobloch K: Differential effects of dofetilide, amiodarone, and class Ic drugs on left and right atrial refractoriness and left atrial vulnerability in pigs. Naunyn Schmiedebergs Arch Pharmacol 2001; 363:166-174. 
17. Knobloch K, Brendel J, Peukert S, Rosenstein B, Busch AE, Wirth KJ: Electrophysiological and antiarrhythmic effects of the novel I(Kur) channel blockers, S9947 and S20951, on left vs. right pig atrium in vivo in comparison with the $\mathrm{I}(\mathrm{Kr})$ blockers dofetilide, azimilide, d,I-sotalol and ibutilide. Naunyn Schmiedebergs Arch Pharmacol 2002; 366: 482-487.

18. Mori K, Hara Y, Saito T, Masuda Y, Nakaya H: Anticholinergic effects of class III antiarrhythmic drugs in guinea pig atrial cells. Different molecular mechanisms. Circulation 1995; $91: 2834-2843$.

19. Watanabe $\mathrm{Y}$, Hara $\mathrm{Y}$, Tamagawa $\mathrm{M}$, Nakaya $\mathrm{H}$ : Inhibitory effect of amiodarone on the muscarinic acetylcholine receptor-operated potassium current in guinea pig atrial cells. J Pharmacol Exp Ther 1996; 279:617-624.

20. Guillemare E, Marion A, Nisato D, Gautier P: Inhibitory effects of dronedarone on muscarinic $\mathrm{K}+$ current in guinea pig atrial cells. J Cardiovasc Pharmacol 2000; 36:802-805.

21. Navarro-Polanco RA, Sanchez-Chapula JA: 4-aminopyridine activates potassium currents by activation of a muscarinic receptor in feline atrial myocytes. J Physiol 1997; 498:663-678.

22. Shi $\mathrm{H}$, Wang $\mathrm{H}$, Wang $\mathrm{Z}$ : Identification and characterization of multiple subtypes of muscarinic acetylcholine receptors and their physiological functions in canine hearts. Mol Pharmacol 1999; 55:497-507.

23. Shi H, Wang H, Li D, Nattel S, Wang Z: Differential alterations of receptor densities of three muscarinic acetylcholine receptor subtypes and current densities of the corresponding $\mathrm{K}+$ channels in canine atria with atrial fibrillation 
induced by experimental congestive heart failure. Cell Physiol Biochem 2004; 14:31-40.

24. Pleumsamran A, Kim D: Membrane stretch augments the cardiac muscarinic K+ channel activity. J Membr Biol 1995; 148:287-297.

25. Courtemanche M, Ramirez RJ, Nattel S: lonic targets for drug therapy and atrial fibrillation-induced electrical remodeling: insights from a mathematical model. Cardiovasc Res 1999; 42:477-489.

26. Duytschaever M, Blaauw $\mathrm{Y}$, Allessie M: Consequences of atrial electrical remodeling for the anti-arrhythmic action of class IC and class III drugs. Cardiovasc Res 2005; 67:69-76.

27. Wijfels MC, Dorland R, Allessie MA: Pharmacologic cardioversion of chronic atrial fibrillation in the goat by class IA, IC, and III drugs: a comparison between hydroquinidine, cibenzoline, flecainide, and d-sotalol. J Cardiovasc Electrophysiol 1999; 10:178-193.

28. Blaauw Y, Gogelein H, Tieleman RG, van Hunnik A, Schotten U, Allessie MA: "Early" class III drugs for the treatment of atrial fibrillation: efficacy and atrial selectivity of AVE0118 in remodeled atria of the goat. Circulation 2004; 110:1717-1724.

29. Blaauw Y, Schotten U, van Hunnik A, Neuberger HR, Allessie MA: Cardioversion of persistent atrial fibrillation by a combination of atrial specific and non-specific class III drugs in the goat. Cardiovasc Res 2007; 75:89-98.

30. Linz DK, Afkham F, Itter G, Rutten H, Wirth KJ: Effect of atrial electrical remodeling on the efficacy of antiarrhythmic drugs: comparison of amiodarone 
with I(Kr)- and I(to)/I(Kur)-blockade in vivo. J Cardiovasc Electrophysiol 2007; 18:1313-1320.

31. Wijfels MC, Kirchhof CJ, Dorland R, Power J, Allessie MA: Electrical remodeling due to atrial fibrillation in chronically instrumented conscious goats: roles of neurohumoral changes, ischemia, atrial stretch, and high rate of electrical activation. Circulation 1997; 96: 3710-3720.

32. Pandit SV, Zlochiver S, Filgueiras-Rama D, et al.: Targeting atrioventricular differences in ion channel properties for terminating acute atrial fibrillation in pigs. Cardiovasc Res 2011; 89:843-851. 
Chapter 5

Renal Sympathetic Denervation Suppresses Postapneic Blood Pressure Rises And Atrial Fibrillation in a Model For Sleep Apnea.

Dominik Linz; Felix Mahfoud; Christian Ukena; Hans-Ruprecht Neuberger; Klaus Wirth; Michael Böhm; Ulrich Schotten.

Hypertension. 2012 Jul;60(1):172-8. 


\section{Abstract}

Background: The aim of this study was to identify the relative impact of adrenergic and cholinergic activity on atrial fibrillation (AF)-inducibility and blood pressure in a model for obstructive sleep apnea (OSA). OSA is associated with sympathovagal dysbalance, AF and postapneic blood pressure rises. Renal denervation (RDN) reduces renal efferent and possibly also afferent sympathetic activity and blood pressure in resistant hypertension.

Methods: The effects of RDN compared to beta-blockade by atenolol on atrial electrophysiological changes, AF-inducibility and blood pressure during obstructive events and on shortening of atrial effective refractory period (AERP) induced by highfrequency stimulation of ganglionated plexi were investigated in 20 anesthetized pigs. Results: Tracheal occlusion with applied negative tracheal pressure (NTP, at -80 mbar) induced pronounced AERP-shortening and increased AF-inducibility in all pigs. RDN but not atenolol reduced NTP-induced AF-inducibility (20\% vs. $100 \%$ at baseline, $\mathrm{p}=0.0001$ ) and attenuated NTP-induced AERP-shortening more than atenolol ( $27 \pm 5 \mathrm{~ms}$ vs. $43 \pm 3 \mathrm{~ms}$ after atenolol, $\mathrm{p}=0.0272)$. Administration of atropine after RDN or atenolol completely inhibited NTP-induced AERP-shortening. AERPshortening induced by high-frequency stimulation of ganglionated plexi was not influenced by RDN, suggesting that changes in sensitivity of ganglionated plexi do not play a role in the antiarrhythmic effect of RDN. Postapneic blood pressure rise was inhibited by RDN and not modified by atenolol.

Conclusions: We showed that vagally mediated NTP-induced AERP-shortening is modulated by RDN or atenolol, which emphasizes the importance of autonomic dysbalance in OSA associated AF. Renal denervation displays antiarrhythmic effects by reducing NTP-induced AERP-shortening and inhibits postapneic blood pressure rises associated with obstructive events. 


\section{Introduction}

Patients with obstructive sleep apnea (OSA) show a high prevalence of atrial fibrillation (AF) ${ }^{1-3}$ and OSA is considered an etiologic factor in the development of hypertension ${ }^{4}$ and in the progress of resistant hypertension ${ }^{5}$. Severe bradycardia and atrioventricular conduction disturbances together with post-apneic blood pressure rises during the arousal are frequently seen in OSA and suggest sympathovagal activation ${ }^{6,7}$. While enhanced vagal tone is known to induce shortening of the atrial effective refractory period (AERP), increased sympathetic tone may increase spontaneous triggered activity both of which, when simultaneously occurring, could induce and maintain $\mathrm{AF}^{8}$. Additionally, repetitive postapneic blood pressure surges may lead to atrial structural changes and thus an arrhythmogenic substrate for AF. Previously, we showed that negative tracheal pressure (NTP) during obstructive respiratory events leads to pronounced shortening of the AERP thereby perpetuating AF 9 . These electrophysiological changes were mainly mediated by increased vagal tone since they were completely inhibited by atropine or bilateral vagotomy. However, less is known about the relative impact of adrenergic and cholinergic activity on AF inducibility and maintenance in OSA.

We hypothesized that modulation of the sympathetic nervous system might reduce AF-susceptibility and postapneic blood pressure rises in OSA. Renal denervation is a new therapeutic approach to reduce sympathetic activity, blood pressure and apneahypopnea index (OSA severity) in resistant hypertension ${ }^{10-13}$. However, the effect of RDN on AF-inducibility and blood pressure during and after obstructive events is unknown. We tested the effect of denervation of the afferent and efferent renal sympathetic nerves previously shown to reduce renal and whole body sympathetic 
activity ${ }^{10-12}$, and compared it to beta-receptor blockade by atenolol on atrial electrophysiological changes and postapneic blood pressure rises in a pig model for OSA. To check whether RDN displays its antiarrhythmic effects by a modulation of sensitivity of the intrinsic cardiac nervous system, we investigated the influence of RDN on sensitivity of right atria to stimulation of autonomic ganglionated plexi.

\section{Methods}

All animal studies were performed in accordance with the guide for the Care and Use of laboratory Animals published by the US National Institutes of Health (NIH Publication No. 85-23, revised 1996).

\section{Experimental model for OSA}

In 15 chest-closed male castrated pigs $(25-30 \mathrm{~kg})$ of the German Landrace (anesthetized with $20 \%$ urethane $(0.8 \mathrm{ml} / \mathrm{kg}$ i.v. load, $0.4 \mathrm{ml} / \mathrm{kg} / \mathrm{h}$ maintenance) and $4 \%$ alpha-chloralose ( $0.4 \mathrm{ml} / \mathrm{kg}$ i.v. load, $0.1 \mathrm{ml} / \mathrm{kg} / \mathrm{h}$ maintenance), a tracheotomy was performed to place an endotracheal tube. This tube was used for tracheal occlusion and to apply different levels of negative tracheal pressures (NTP) by a negative pressure device ${ }^{9,14}$. The Mueller maneuver (forced inspiration against airway obstruction after deep exspiration generating a negative pressure of about 40 $\mathrm{mmHg}$ ) is used in the clinical setting to simulate conditions, particularly negative thoracic pressure, during obstructive sleep apnea ${ }^{15}$. As a modification of this maneuver, we applied NTP at -80 mbar during tracheal occlusion corresponding to NTPs found in patients with OSA ${ }^{6,7,15,16}$. 
Blood pressure was measured by a TIP-catheter (Millar PC 350; Millar Instruments, Houston, Texas, USA) in the femoral artery. Bipolar body surface ECG was recorded using subcutaneous needle electrodes in the classical lead II arrangement. The ECGs were analyzed concerning heart rate and conduction times according to the AHA/ACCF/HRS Recommendations for the Standardization and Interpretation of the Electrocardiogram before and after RDN or atenolol. The QT interval was corrected using the Bazett formula $(\mathrm{QTc}=\mathrm{QT} / \sqrt{ } \mathrm{RR})$. Blood gas analyses $\left(\mathrm{pO}_{2}, \mathrm{pCO}_{2}, \mathrm{pH}, \mathrm{O}_{2^{-}}\right.$ saturation) were performed directly before and at 2 minutes of each tracheal occlusion with applied NTP. In seven animals, both kidneys were approached through bilateral retroperitoneal flank incisions. Both kidneys were surgically denervated by cutting all visible nerves in the area of the renal hilus and by stripping approximately $1 \mathrm{~cm}$ of the adventitia from the renal artery. The area was then moistened with a $20 \%$ phenol/ethanol solution for $10-15$ min. Left renal flow was measured with a doppler flow probe (transit time flowmeter module system from Transonic Systems Inc.; Germany) positioned on the blood vessels. Following RDN, the animals were allowed to re-equilibrate for 1.5 hours. Significant reduction of the reproducible post-apneic BP-rise and the absence ( $<5 \%$ change) of a decrease in renal blood flow induced by tracheal occlusion with applied NTP were taken as evidence of the completeness of RDN. Sham

surgical procedure with kidney exposition without renal denervation was performed in three additional pigs serving as a time control. Results for vehicle-time controls for atenolol and atropin have been published in ${ }^{9,14}$. Vehicle application did not change NTP-induced electrophysiological parameters or hemodynamics within 4 hours.

Five additional pigs $(35-41 \mathrm{~kg})$ were anesthetized with pentobarbital ${ }^{17}$ and a right thoracotomy at the 4th intercostal space provided access to the right lung and atria through a pericardiotomy. At the caudal end of the sinus node between the right 
superior and inferior pulmonary vein, a plaque electrode containing 2 electrodes was sutured on the fat pad (anterior right ganglionated plexi (GP)). For electrical GPstimulation, the following stimulation parameters were used: frequency: $20 \mathrm{~Hz}$, stimulus duration: $0.1 \mathrm{~ms}$, voltage: $0.1-5$ volt. GP-stimulation, which slowed heart rate by approximately $50 \%$ at baseline, was applied before and $1.5 \mathrm{~h}$ after bilateral RDN.

\section{Electrophysiological examinations}

During normal breathing, atrial effective refractory period (AERP) was measured at a basic cycle length (BCL) of 400,300 and $240 \mathrm{~ms}$ before and after RDN or atenolol. Additionally, AERP measurements at a representative basic cycle length $(B C L)$ of $300 \mathrm{~ms}$ and AF-inducibility were performed before, during, and after tracheal occlusion. An extended description of electrophysiological examinations is available as an online Data Supplement (please see http://hyper.ahajournals.org).

\section{Drugs}

Atenolol (3mg/ $/ \mathrm{kg})$ and atropine was dissolved in $0.5 \mathrm{ml}$ dimethyl sulfoxide (DMSO) and $2.5 \mathrm{ml}$ of polyethyleneglycol (PEG) 400. All solutions were injected over 5 minutes. Vehicle as a control was injected 15 min before the second baseline NTPrun in all animals of group 1. Atenolol was used in a concentration which resulted in a maximal heart rate reduction and AERP-prolonging effect in previous studies ${ }^{9,14}$.

\section{Experimental design}

The electrophysiological study protocol (EP) and the experimental design are depicted in figure 1. AERP-measurements were performed and AF-inducibility was investigated during normal breathing and at every 30 seconds during 2 minutes of tracheal occlusion with applied NTP at -80 mbar. Recovery of the AERP was shown by AERP-measurement at $15 \mathrm{~min}$ between the different tracheal occlusion 
maneuvers. After the first run, both kidneys were approached through bilateral retroperitoneal flank incisions in group 1 and vehicle was given to all animals of group 2. Bilateral RDN was performed in group 1 and atenolol was given to all animals of group 2. Two additional runs of the electrophysiological study protocol were performed in exactly the same manner 15 min and 1.5 hours after RDN and 15 min after atenolol. In group 3, AERP-shortening induced by high frequency stimulation of GPs was investigated at baseline and 1.5 hours after RDN. Sham surgical procedure was performed in three additional pigs serving as a time control.

\section{Statistics}

Data are presented as mean \pm SEM. For comparisons of single repeated measures only, a paired Student's t-test was used. For multiple repeated measures comparisons with the same baseline, repeated-measures two-way ANOVA was used, followed by Dunnet's test to compare individual mean differences if ANOVA was significant. A p value below 0.05 was considered significant. For all statistical calculations software Everstat V5 based on SAS 8 was used. 


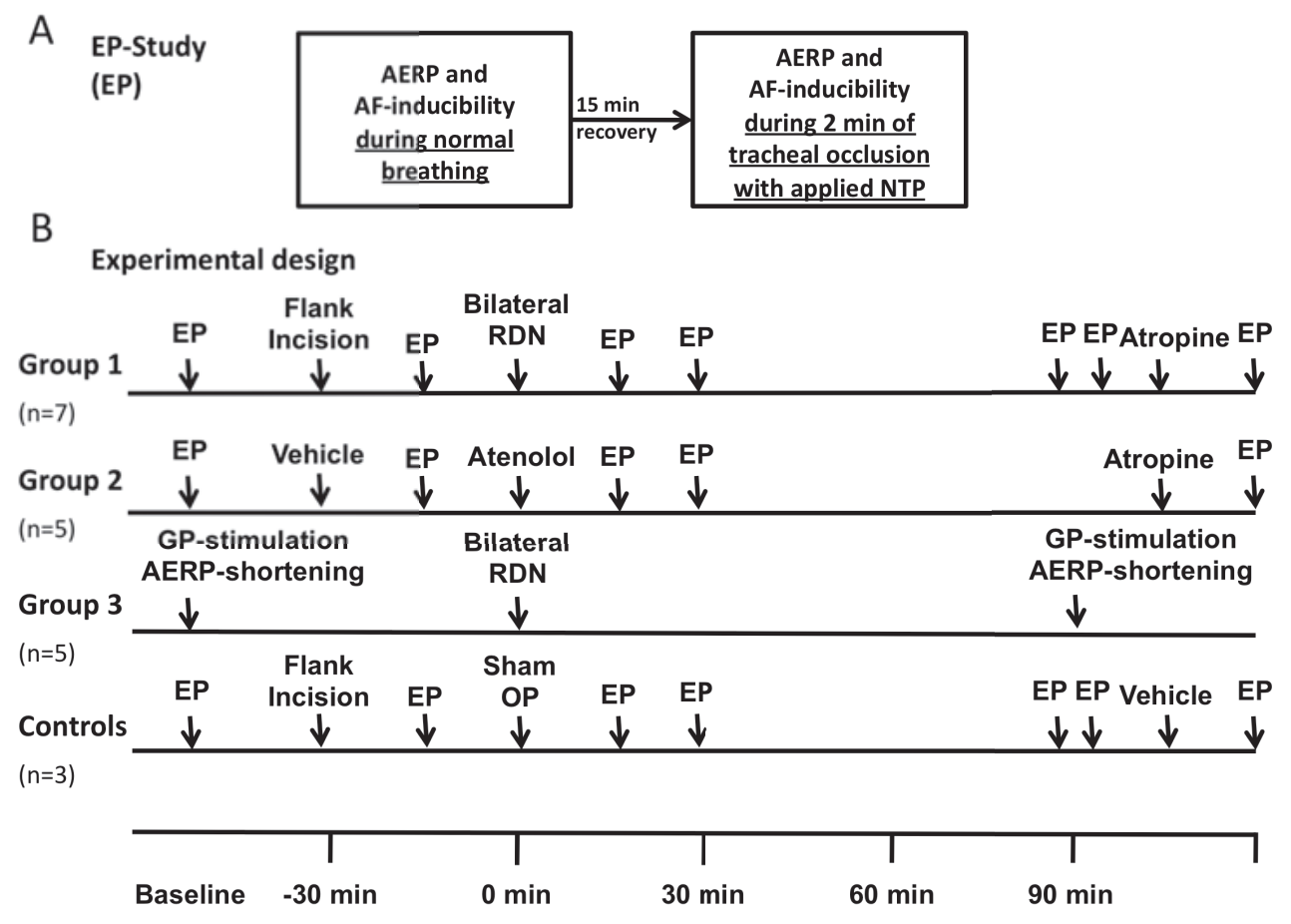

Figure 1: Experimental Design Flow Chart: (A) A complete electrophysiological study protocol (EP-study, EP) included atrial effective refractory period (AERP)- and atrial fibrillation (AF)-inducibility-measurement during normal breathing and every 30 seconds during 2 minutes of tracheal occlusion with applied negative tracheal pressure (NTP) at -80 mbar. (B) Experimental design. Group 1: Effect of bilateral renal denervation (RDN) on NTP-induced AERP-shortening and AF-inducibility. Group 2: Effect of atenolol on NTP-induced AERP-shortening and AF-inducibility. Group 3: Effect of bilateral RDN on AERP-shortening induced by high frequently stimulation of ganglionated plexi (GP). 


\section{Results}

\section{Electrophysiological effects of RDN and atenolol during normal breathing}

Figure 2 shows the effects of bilateral RDN (A) and atenolol (B) on AERP during normal breathing. Atenolol increased the AERP by $27.2 \pm 3.2 \mathrm{~ms}$ at $300 \mathrm{~ms} \mathrm{BCL}$ $(p=0.0001)$. RDN did not influence AERP significantly during normal breathing. During normal breathing, PQ-interval was prolonged and heart rate was reduced by RDN and atenolol. P-wave duration, QRS-duration and cQT-time were not modified by RDN or atenolol. (figure S1, please see the online Data Supplement).

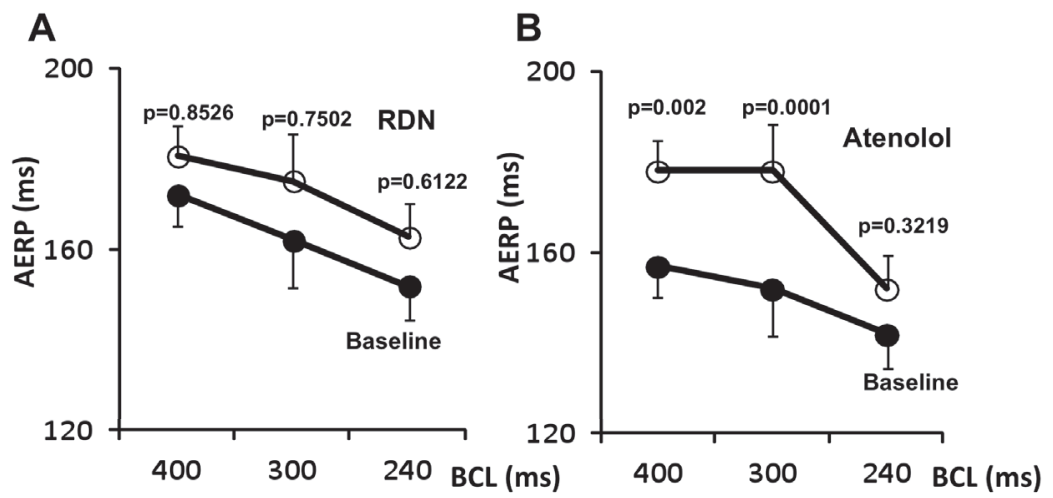

Figure 2: Changes in Atrial Effective Refractory Period During Normal Breathing: Effect of renal denervation (RDN) (A) and atenolol $(3 \mathrm{mg} / \mathrm{kg})(B)$ on atrial effective refractory period (AERP) at different basic cycle lengths (BCL: $400 \mathrm{~ms}, 300$ $\mathrm{ms}$ and $240 \mathrm{~ms}$ ) (filled symbols: baseline; white symbols: after treatment). 


\section{Effect of RDN and atenolol on NTP-induced AERP-shortening}

Tracheal occlusion without applied NTP did not change the AERP, neither before nor after RDN or atenolol. In contrast, the application of NTP during tracheal occlusion induced a pronounced AERP-shortening (from $167.8 \pm 7.9 \mathrm{~ms}$ to $105.2 \pm 10.6 \mathrm{~ms}$, $\mathrm{p}=0.0001)$. In 3 control animals, repetitive full electrophysiological study protocols including NTP-maneuvers and sham operation were conducted over the time frame similar to those of the treated animals. NTP-induced changes in electrophysiology and recovery at each repetition over time were constant. In figure 3 , the effect of RDN and atenolol followed by atropine on NTP-induced AERP-shortening during tracheal occlusion is shown.

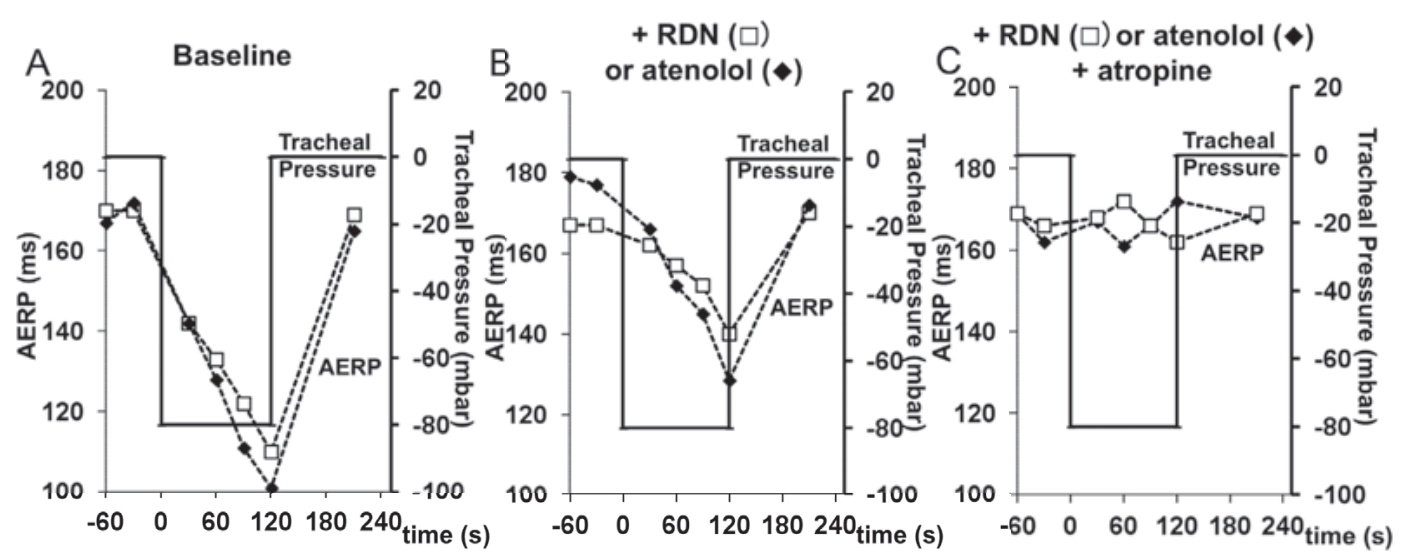

Figure 3: Changes in Atrial Effective Refractory Period During Tracheal Occlusion: Changes in atrial effective refractory period (AERP) (dotted line, left ordinate) during tracheal occlusion with applied negative tracheal pressure (NTP) at 80 mbar for 120 seconds (continuous line, right ordinate) before (A) and after renal denervation (RDN) or atenolol (B) followed by atropine (C). Representative time curves of a pig of group 1 (RDN followed by atropine, white squares) and a pig of group 2 (atenolol followed by atropine, black diamonds) are shown. 
RDN reduced NTP-induced AERP-shortening (AERP-shortening: $27 \pm 5$ ms after RDN vs. $43 \pm 3$ ms after atenolol, $p=0.0272$ ) (figure $4 \mathrm{~A}$ ) and NTP-induced MAP-shortening at $70 \%$ of repolarisation (MAP-shortening: $22 \pm 2.1 \mathrm{~ms}$ after RDN vs. $47 \pm 7.2 \mathrm{~ms}$ after atenolol, $\mathrm{p}=0.0024$ ) (figure $4 \mathrm{~B}$ ) more than atenolol. However, atropine applied after the administration of atenolol or RDN completely inhibited NTP-induced AERPshortening and MAP-shortening.
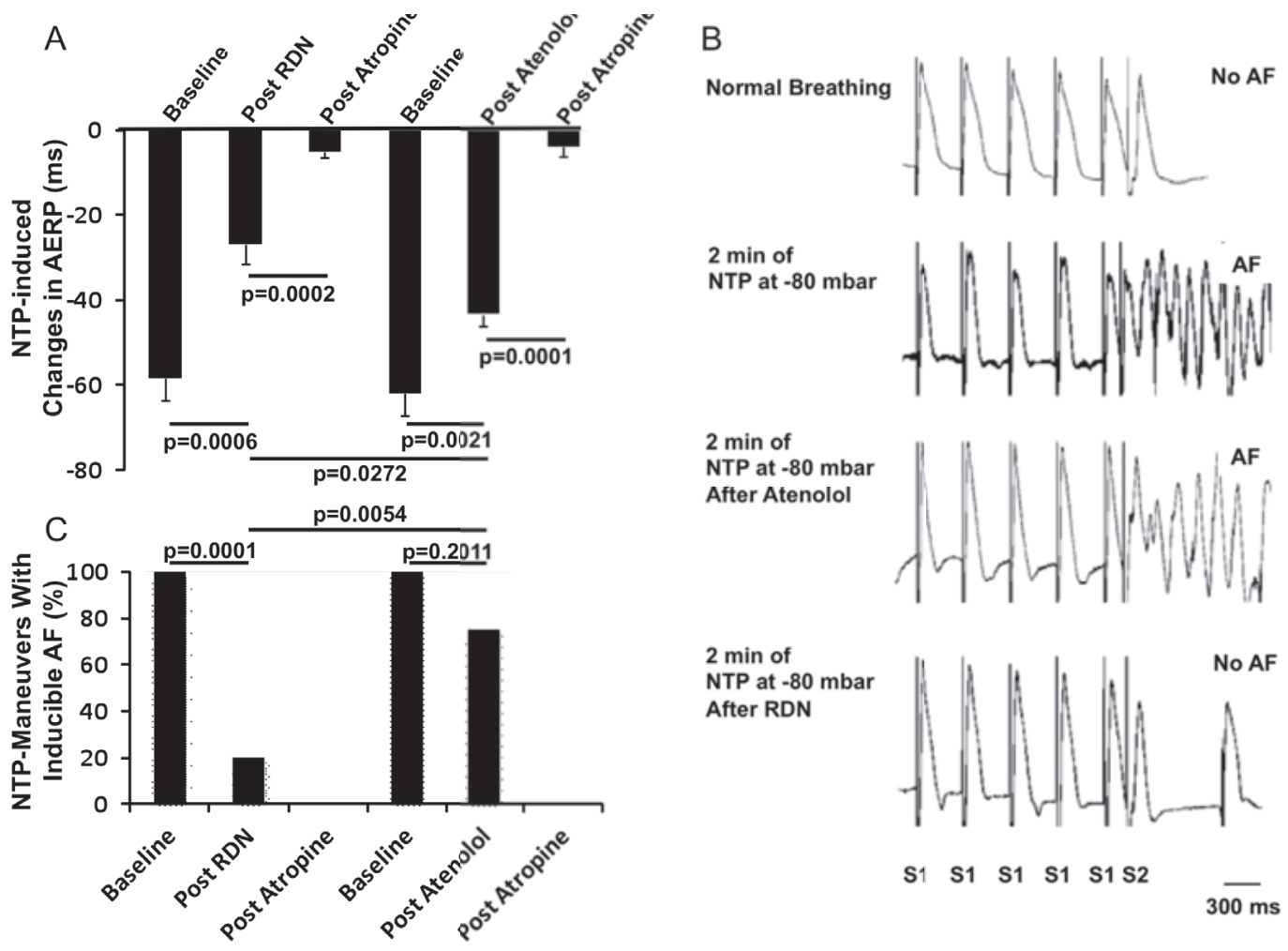

Figure 4: Electrophysiological Changes During Tracheal Occlusion: (A) Effect of renal denervation (RDN) and atenolol followed by atropine on changes in atrial effective refractory period (AERP) induced by tracheal occlusion with applied negative tracheal pressure (NTP) at -80 mbar (ordinate). (B) Representative atrial multiple action potential (MAP) recordings during AERP-measurements before and after RDN or atenolol. (C) Percent of tracheal occlusions with inducible atrial fibrillation (AF) (ordinate) and effect of RDN and atenolol followed by atropine. 


\section{Effect of RDN and atenolol on NTP-induced AF-inducibility}

AF was not inducible by a single premature stimulus during the AERP-measurement procedure during normal breathing. NTP-induced AERP-shortening and MAPshortening were associated with increased inducibility of AF by a single premature stimulus during the AERP-measurement from 0\% during normal breathing to $100 \%$ during tracheal occlusion with applied NTP $(p=0.0001)$. In figure $4 \mathrm{C}$, the effect of RDN and atenolol on NTP-induced AF-inducibility is shown. RDN inhibited NTPinduced AF-inducibility. Atenolol did not reduce NTP-induced AF-inducibility significantly but inducible AF-duration was shortened (27 s vs. $122 \mathrm{~s}$ at baseline, $\mathrm{p}=0.0037)$. After administration of atropine following RDN or atenolol no episode of AF was inducible.

\section{Effect of RDN on sensitivity of right atria to stimulation of autonomic ganglionated plexi}

RDN did not influence AERP-shortening induced by GP-stimulation as a result of programmed electrical stimulation (AERP-shortening: $51 \pm 4 \mathrm{~ms}$ vs. $56 \pm 5 \mathrm{~ms}$ after RDN, $p=0.2915)$.

\section{Effect of RDN and atenolol on postapneic blood pressure rises and blood gases}

Postapneic changes in blood pressure (BP) after bilateral RDN or atenolol are shown in figure 5. RDN abolished post-apneic BP surge. Suppression of the post-apneic BP rise was not observed after atenolol. NTP-induced changes in blood gases were not modified by RDN or atenolol (table S1, please see the online Data Supplement). 


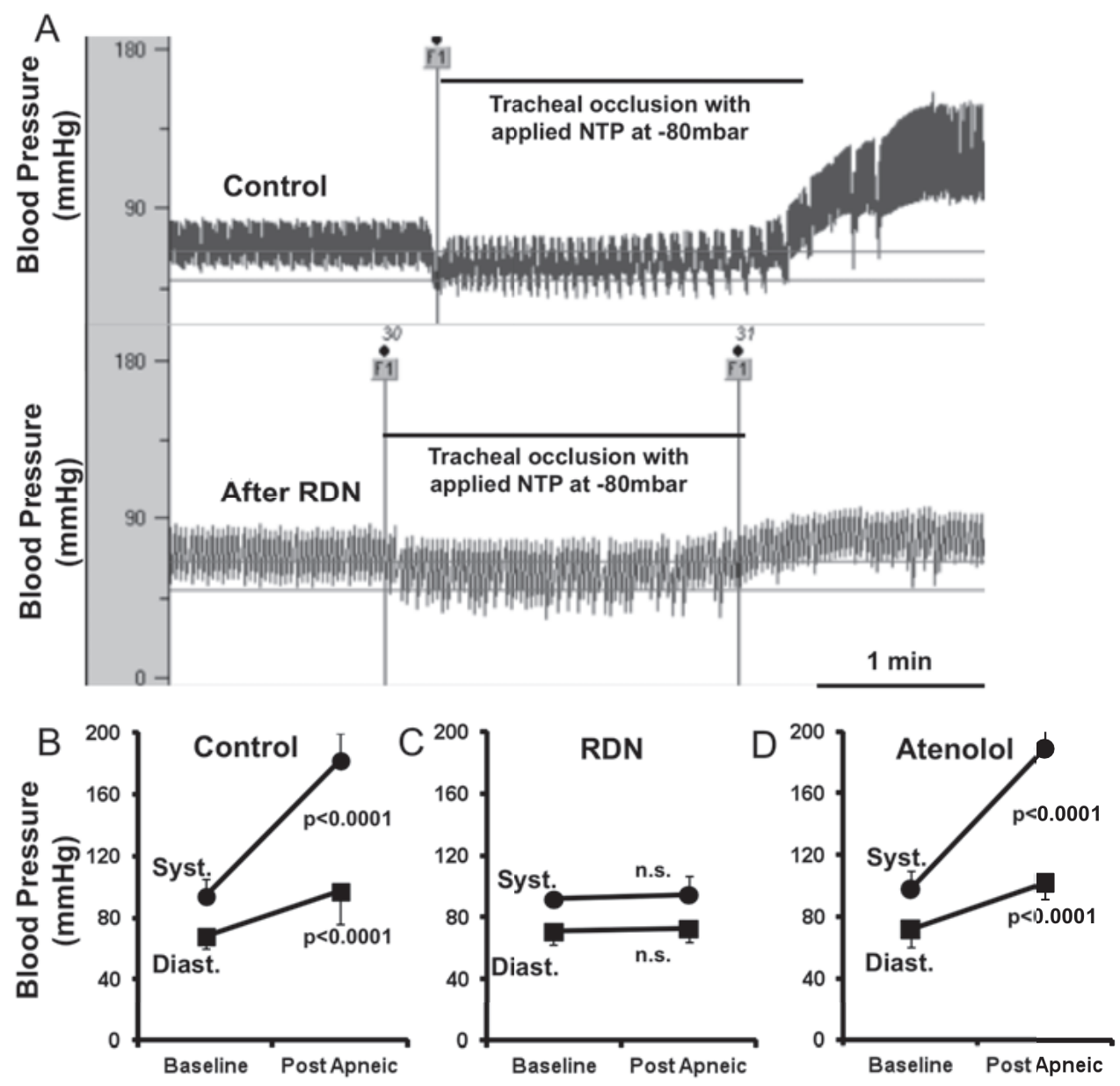

Figure 5: Postapneic Blood Pressure Rise: Original registration of postapneic blood pressure changes in a pig before (control) and 1.5 hours after renal denervation (RDN) (A). Postapneic blood pressure changes (ordinate) before (control) (B) and 1.5 hours after RDN (C) and 15 minutes after atenolol (D). 


\section{Discussion}

In this study we showed that postapneic blood pressure rises were abolished by RDN but not by atenolol. Arrhythmogenic electrophysiological changes during tracheal occlusion with applied NTP, mainly characterized by a pronounced shortening of AERP, can be modified by RDN and atenolol. However, AF-inducibility was inhibited by RDN only and not by atenolol. AERP-shortening induced by high-frequency stimulation of ganglionated plexi (GP) was not modified by RDN.

Several observations suggest that the autonomic nervous system plays an important role in both the initiation and the maintenance of $A F$ in humans. Vagal nerve stimulation and acetylcholine infusion shortens the AERP ${ }^{8}$. By contrast, activation of sympathetic activity showed variable effects on AERP $8,18,19$. Interestingly, studies in lone AF patients and in animal models of both intermittent rapid atrial pacing and congestive heart failure have indicated that AF onset is associated with simultaneous sympathovagal activation rather than with an increase in vagal or sympathetic drive alone ${ }^{20-23}$. Previously we have shown increased AF-susceptibility and AF-duration caused by pronounced AERP-shortening in a pig model for OSA ${ }^{9,14}$. Negative thoracic pressure (NTP) during breathing attempts against the obstructed upper airways was identified to be a trigger for AERP-shortening. AERP-shortening was mediated by pronounced vagal activation since it was completely inhibited by atropine and bilateral vagotomy. However, increased negative thoracic pressure, hypoxia and hypercapnia during obstructive apnea are associated with severe bradycardia and atrioventricular block together with post-apneic blood pressure rises suggesting simultaneous sympathovagal activation ${ }^{6,7}$. Direct recordings of muscle sympathetic nerve activity showed increased sympathetic activation during apneic 
episodes in OSA patients ${ }^{16}$ or in apnea divers ${ }^{24}$. Sympathetic activity may increase profoundly during the application of negative tracheal pressure resulting in the pronounced postapneic blood pressure rise.

The sympathetic nervous system can be modulated by blockade of beta-receptors and RDN in humans ${ }^{10-12}$. NTP-induced AERP- and MAP-shortening were significantly reduced by RDN and atenolol. This indicates that sympathetic modulation affects NTP-associated electrophysiological changes. Atropine after RDN or atenolol completely inhibited NTP-induced AERP- and MAP-shortening, showing that vagal activation beyond sympathetic activation is one significant determinant of NTP-induced shortening of atrial refractoriness.

The suppression of vagally mediated AERP- and MAP-shortening during obstructive events by RDN or atenolol indicates an interaction between the acetylcholine (ACh)induced AERP-shortening and the reduced activation of the sympathetic nervous system. Consistently, Sharifov et al. ${ }^{25}$ showed that ACh-mediated AF was facilitated by isoproterenol, which decreased the threshold of $\mathrm{ACh}$ concentration for $\mathrm{AF}$ induction and increased AF duration. Patterson et al. ${ }^{26}$ showed that the synergistic action of both the sympathetic and the parasympathetic neurotransmitter are required to initiate rapid ectopic discharges in superfused pulmonary vein preparations. Indeed, we observed that AF was induced by spontaneous atrial premature beats during tracheal occlusion in some animals, which may represent the occurrence of ectopic discharges triggering AF. However, this spontaneous induction of AF was too rare for a systematic evaluation. 
RDN but not atenolol reduced NTP-induced AF-inducibility. Catheter-based denervation of the kidneys by ablation of the efferent sympathetic and afferent somatic fibers has been shown to be effective in reducing both renal and whole-body norepinephrine spillover and muscle sympathetic nerve activity ${ }^{10-12}$. Altering the signals from the kidney to the hypothalamus is expected to impact down-stream organ systems including the vasculature and the heart ${ }^{27}$. Catheter-based RDN in patients with resistant hypertension effectively reduced blood pressure 10-12, improved insulin resistance ${ }^{28}$ and sleep apnea severity ${ }^{13}$. Interestingly, NTPinduced post-apneic blood pressure surges were attenuated by RDN suggesting a sympatho-inhibitory effect. However, similar effects have not been reported for betablocker treatment clearly indicating a different mechanism of action. Possibly, RDN might additionally modify alpha- adrenoceptors pathways which are not influenced by atenolol. Interestingly, activation of the intrinsic cardiac nervous system results in atrial arrhythmias that involve intrinsic cardiac neuronal alpha-adrenoceptors ${ }^{29}$.

RDN resulted in a more pronounced inhibition of NTP-induced AERP-shortening compared to atenolol, which might explain the superior antiarrhythmic effect of RDN compared to atenolol. Changes in blood gases during tracheal occlusion or $p$-wave duration as a marker of atrial conduction were neither modified by RDN nor by atenolol suggesting an antiarrhythmic effect independent of changes in blood gases or atrial conduction. RDN and atenolol prolonged PQ-interval and reduced heart rate which may represent the modulation of sympathetic activity. First supportive data for potential antiarrhythmic effect of acute RDN has recently been published by Ukena et al. ${ }^{30}$. They reported reduced ventricular tachyarrhythmias in patients with electrical storm and congestive heart failure after catheter-based RDN. However, data from clinical studies powered to detect these effects are lacking. 
Importantly, long-term OSA has been shown to be associated with significant atrial remodeling characterized by atrial enlargement, reduction in voltage, site-specific and widespread conduction abnormalities, and longer sinus node recovery in humans ${ }^{31}$. Apart the described antiarrhythmic electrophysiological effects of RDN, RDN might be able to attenuate the development of an atrial proarrhythmic substrate by inhibiting postapneic blood pressure surges as shown in this study. Additionally, RDN has been proven to reduce OSA severity in OSA-patients ${ }^{13}$.

We tested, whether parts of the antiarrhythmic effects of RDN can be attributed to a modulation of the sensitivity of the intracardiac autonomic nervous system containing ganglionated plexi (GPs). GPs may modulate the interactions between extrinsic and intrinsic cardiac autonomic nervous system ${ }^{32}$ and contain efferent cholinergic and adrenergic neurons influencing the atrial myocardium. GP-ablation combined with a pulmonary vein ablation procedure has been shown to further improve success rates of reversion in paroxysmal AF-patients ${ }^{33}$. In a dog model for central sleep apnea GPablation inhibited AF-inducibility ${ }^{34}$. In this study, AERP-shortening induced by highfrequency stimulation of GPs was not modified by RDN. This suggests that changes in sensitivity of GPs do not play a role in the antiarrhythmic effect of RDN.

\section{Limitations}

Spontaneous induction of $\mathrm{AF}$ was too rare for a systematic evaluation. Therefore we applied a premature beat during tracheal occlusion with applied NTP to induce AF. AERP-measurement in the left atrium and the investigation of the spatial distribution of refractoriness, of inhomogeneity in conduction and conduction velocity in the 
atrium would have required thoracotomy. In pigs with thoracotomy application of NTP would not be possible. Additionally, catheter based renal nerve ablation in humans is possibly not complete, as indicated by a norepinephrine spillover reduction by only about $50 \%{ }^{10}$. We performed complete surgical renal nerve ablation in this study. The precise measurement of sympathetic activity and GP-activation during and after obstructive events deserves further studies.

\section{Conclusions and Perspectives}

RDN reduced postapneic blood pressure rises, which may attenuate OSA-associated structural atrial arrhythmogenic remodeling. Additionally, vagally mediated NTPinduced AERP- and MAP-shortening is modulated by RDN or atenolol, which emphasizes the importance of autonomic dysbalance in OSA associated AF. RDN but not atenolol reduced AF-inducibility. RDN displays antiarrhythmic effects independent of a modulation of sensitivity of intracardiac ganglionated plexi, suggesting that ganglionated plexi do not play a relevant role in the antiarrhythmic effect of RDN. Modulation of the autonomic nervous system by RDN might be useful to reduce atrial arrhytmogenesis and deserves to be tested in clinical studies and in AF-models for structural and/or electrical atrial remodelling. 


\section{References}

1. Kanagala R, Murali NS, Friedman PA, Ammash NM, Gersh BJ, Ballman KV, Shamsuzzaman AS, Somers VK. Obstructive sleep apnea and the recurrence of atrial fibrillation. Circulation. 2003;107:25892594.

2. Gami AS, Hodge DO, Herges RM, Olson EJ, Nykodym J, Kara T, Somers VK. Obstructive sleep apnea, obesity, and the risk of incident atrial fibrillation. J Am Coll Cardiol. 2007;49:565-571.

3. Jongnarangsin K, Chugh A, Good E, Mukerji S, Dey S, Crawford T, Sarrazin JF, Kuhne M, Chalfoun N, Wells D, Boonyapisit W, Pelosi F Jr, Bogun F, Morady F, Oral H. Body mass index, obstructive sleep apnea, and outcomes of catheter ablation of atrial fibrillation. J Cardiovasc Electrophysiol. 2008;19:668-672.

4. Peppard PE, Young T, Palta M, Skatrud J. Prospective study of the association between sleep-disordered breathing and hypertension. $N$ Engl J Med. 2000;342:1378-1384.

5. Logan AG, Perlikowski SM, Mente A, Tisler A, Tkacova R, Niroumand M, Leung RS, Bradley TD. High prevalence of unrecognized sleep apnoea in drug-resistant hypertension. J Hypertens. 2001;19:22712277.

6. Kohler M, Stradling JR. Mechanisms of vascular damage in obstructive sleep apnea. Nat Rev Cardiol. 2010;7:677-685. 
7. Kasai T, Bradley TD. Obstructive sleep apnea and heart failure: pathophysiologic and therapeutic implications. J Am Coll Cardiol. 2011;57:119-127.

8. Schotten U, Verheule S, Kirchhof P, Götte A. Pathophysiological mechanisms of atrial fibrillation: a translational appraisal. Physiol Rev. 2011;91:265-325.

9. Linz D, Schotten U, Neuberger HR, Böhm M, Wirth K. Negative Tracheal Pressure During Obstructive Respiratory Events Promotes Atrial Fibrillation by Vagal Activation. Heart Rhythm. 2011;8:1436-1443.

10. Krum H, Schlaich M, Whitbourn R, Sobotka PA, Sadowski J, Bartus K, Walton A, Sievert H, Thambar S, Abraham WT, Esler M. Catheterbased renal sympathetic denervation for resistant hypertension: a multicentre safety and proof-of-principle cohort study. Lancet. 2009;373:1275-1281.

11. Schlaich MP, Sobotka PA, Krum H, Lambert E, Esler MD. Renal sympathetic-nerve ablation for uncontrolled hypertension. $N$ Engl $J$ Med. 2009;361:932-934.

12. Esler MD, Krum H, Sobotka PA, Schlaich MP, Schmieder RE, Böhm M. Renal sympathetic denervation in patients with treatment-resistant hypertension (The Symplicity HTN-2 Trial): a randomised controlled trial. Lancet. 2010;376:1903-1909.

13. Witkowski A, Prejbisz A, Florczak E, Kadziela J, Sliwinski P, Bielen P, Michalowska I, Kabat M, Warchol E, Januszewicz M, Narkiewicz K, 
Somers VK, Sobotka PA, Januszewicz A. Effects of renal sympathetic denervation on blood pressure, sleep apnea course, and glycemic control in patients with resistant hypertension and sleep apnea. Hypertension. 2011;58:559-565.

14. Linz D, Schotten U, Neuberger HR, Böhm M, Wirth K. Combined blockade of early and late activated atrial potassium currents suppresses atrial fibrillation in a pig model of obstructive apnea. Heart Rhythm. 2011;8:1933-1939.

15. Orban M, Bruce CJ, Pressman GS, Leinveber P, Romero-Corral A, Korinek J, Konecny T, Villarraga HR, Kara T, Caples SM, Somers VK. Dynamic changes of left ventricular performance and left atrial volume induced by the mueller maneuver in healthy young adults and implications for obstructive sleep apnea, atrial fibrillation, and heart failure. Am J Cardiol. 2008;102:1557-1561.

16. Parish JM, Somers VK. Obstructive sleep apnea and cardiovascular disease. Mayo Clin Proc. 2004;79:1036-1046.

17. Linz DK, Afkham F, Itter G, Rütten H, Wirth KJ. Effect of atrial electrical remodeling on the efficacy of antiarrhythmic drugs: comparison of amiodarone with I(Kr)- and I(to)/IKur-blockade in vivo. J Cardiovasc Electrophysiol. 2007;18:1313-1320.

18. Inoue H, Zipes DP. Changes in atrial and ventricular refractoriness and in atrioventricular nodal conduction produced by combinations of vagal and sympathetic stimulation that result in a constant spontaneous sinus cycle length. Circ Res. 1987;60:942-951. 
19. Liang BT, Frame LH, Molinoff PB. Beta 2-adrenergic receptors contribute to catecholamine-stimulated shortening of action potential duration in dog atrial muscle. Proc Natl Acad Sci U S A. 1985;82:45214525 .

20. de Vos CB, Nieuwlaat R, Crijns HJ, Camm AJ, LeHeuzey JY, Kirchhof CJ, Capucci A, Breithardt G, Vardas PE, Pisters R, Tieleman RG. Autonomic trigger patterns and anti-arrhythmic treatment of paroxysmal atrial fibrillation: data from the Euro Heart Survey. Eur Heart $\mathrm{J}$. 2008;29:632-639.

21. Ogawa M, Zhou S, Tan AY, Song J, Gholmieh G, Fishbein MC, Luo H, Siegel RJ, Karagueuzian HS, Chen LS, Lin SF, Chen PS. Left stellate ganglion and vagal nerve activity and cardiac arrhythmias in ambulatory dogs with pacing-induced congestive heart failure. J Am Coll Cardiol. 2007;50:335-343.

22. Tan AY, Zhou S, Ogawa M, Song J, Chu M, Li H, Fishbein MC, Lin SF, Chen LS, Chen PS. Neural mechanisms of paroxysmal atrial fibrillation and paroxysmal atrial tachycardia in ambulatory canines. Circulation. 2008;118:916-925.

23. Piccirillo G, Ogawa M, Song J, Chong VJ, Joung B, Han S, Magri D, Chen LS, Lin SF, Chen PS. Power spectral analysis of heart rate variability and autonomic nervous system activity measured directly in healthy dogs and dogs with tachycardia-induced heart failure. Heart Rhythm. 2009;6:546-552. 
24. Heusser K, Dzamonja G, Breskovic T, Steinback CD, Diedrich A, Tank J, Jordan J, Dujic Z. Sympathetic and cardiovascular responses to glossopharyngeal insufflation in trained apnea divers. J Appl Physiol. 2010. 109:1728-1735.

25. Sharifov OF, Fedorov VV, Beloshapko GG, Glukhov AV, Yushmanova AV, Rosenshtraukh LV. Roles of adrenergic and cholinergic stimulation in spontaneous atrial fibrillation in dogs. $J$ Am Coll Cardiol. 2004;43:483-490.

26. Patterson E, Lazzara R, Szabo B, Liu H, Tang D, Li YH, Scherlag BJ, Po SS. Sodium-calcium exchange initiated by the $\mathrm{Ca} 2+$ transient: an arrhythmia trigger within pulmonary veins. J Am Coll Cardiol. 2006;47:1196-1206.

27. Sobotka PA, Mahfoud F, Schlaich MP, Hoppe UC, Böhm M, Krum H. Sympatho-renal axis in chronic disease. Clin Res Cardiol. 2011;100:1049-1057.

28. Mahfoud F, Schlaich M, Kindermann I, Ukena C, Cremers B, Brandt MC, Hoppe UC, Vonend O, Rump LC, Sobotka PA, Krum H, Esler M, Böhm M. Effect of renal sympathetic denervation on glucose metabolism in patients with resistant hypertension: a pilot study. Circulation. 2011;123:1940-1946.

29. Richer LP, Vinet A, Kus T, Cardinal R, Ardell JL, Armour JA. AlphaAdrenoceptor blockade modifies neurally induced atrial arrhythmias. Am J Physiol Regul Integr Comp Physiol. 2008. 295: R1175-R1180. 
30. Ukena C, Bauer A, Mahfoud F, Schreieck J, Neuberger HR, Eick C, Sobotka PA, Gawaz M, Böhm M. Renal sympathetic denervation for treatment of electrical storm: first-in-man experience. Clin Res Cardiol. 2011;101:63-67.

31. Dimitri H, Ng M, Brooks AG, Kuklik P, Stiles MK, Lau DH, Antik N, Thornton A, Saint DA, McEvoy D, Antic R, Kalman JM, Sanders P. Atrial remodeling in obstructive sleep apnea: Implications for atrial fibrillation. Heart Rhythm. 2011;9:321-327.

32. Hou Y, Scherlag BJ, Lin J, Zhang Y, Lu Z, Truong K, Patterson E, Lazzara R, Jackman WM, Po SS. Ganglionated plexi modulate extrinsic cardiac autonomic nerve input: effects on sinus rate, atrioventricular conduction, refractoriness, and inducibility of atrial fibrillation. J Am Coll Cardiol. 2007;50:61-68.

33. Po S, Nakagawa H, Jackman WM. Localization of left atrial ganglionated plexi in patients with atrial fibrillation. $J$ Cardiovasc Electrophysiol. 2009;20:1186-1189.

34. Ghias M, Scherlag BJ, Lu Z, Niu G, Moers A, Jackman WM, Lazzara R, Po SS. The role of ganglionated plexi in apnea-related atrial fibrillation. J Am Coll Cardiol. 2009;54:2075-2083. 
Chapter 6

Zooming in on a Focus: High Density Mapping of the Interaction between Ectopy and Re-entry during

\section{Atrial Fibrillation}

Dominik Linz (1,2), Jens Eckstein (1), Bart Maesen (1), Arne v. Hunnik (1), Marion Kuiper (1), Stef Zeemering (1), Sander Verheule (1), Ulrich Schotten (1). 


\section{Abstract}

Background: The contribution of ectopic focal discharges to the perpetuation of atrial fibrillation (AF) is debated. The interaction of ectopic focal discharges with reentry mechanisms during $A F$ is unknown and criteria for the identification of ectopy during $\mathrm{AF}$ have not been developed.

Methods: In open chest experiments in goats $(n=6)$, stationary ectopic focal discharges were elicited by topical application of aconitine (ACO) on the epicardial surface. AF was induced by burst stimulation of the atria. We performed high density endo-epicardial mapping to investigate the interaction of focal tachycardia induced by the topical epicardial application of ACO with activation pattern during AF.

Results: The topical application of ACO-crystals on the left atrium in the middle of the mapping area induced rapid focal discharges with radial spread of activation exactly at the place of ACO-application (ACO cycle length (CL): $242 \pm 15 \mathrm{~ms}$ ). At the site of earliest activation local electrograms did not show R-waves. At the same site, atrial effective refractory period (BCL=200ms: $135 \pm 2$ ms vs. $135 \pm 4$ ms, n.s.) was unchanged. During overdrive stimulation local conduction velocity (CV) was also not affected by the topical application of aconitine $(80 \pm 3 \mathrm{~cm} / \mathrm{s}$ vs. $80 \pm 3 \mathrm{~cm} / \mathrm{s}$, n.s.). However, episodes of burst-induced AF lasted longer than before aconitine application ( $470 \pm 85 \mathrm{sec}$. vs. $26 \pm 19$ sec., $\mathrm{p}<0.01$ ) while $\mathrm{AFCL}$ was unchanged (133 $\pm 5 \mathrm{~ms}$ vs. $134 \pm 5 \mathrm{~ms}$, n.s.). During AF the rate of breakthrough was more than 10fold higher $(1.14 \pm 0.14$ per cycle vs. $0.08 \pm 0.03$ per cycle before aconitine, $p<0.01)$. However, breakthroughs occurred wide-spread throughout the mapping area, remote from the site of ACO-application. The mean of epicardial coupling intervals of breakthroughs and the mean of AFCL did not differ significantly (131 \pm 3 ms vs. 131 \pm 5 ms, n.s.). More than $80 \%$ of all local electrograms at the site of earliest activation of breakthroughs showed R-waves, but only $44 \%$ of them could be explained by 
transmural conduction. Flecainide inhibited ACO-induced rapid focal discharges at the site of ACO-application and reduced inducibility of AF.

Conclusions: The existence of a single localized site with high propensity to ectopy causes a pronounced increase in AF stability and in the breakthrough rate during AF despite the fact that the cycle length of the ectopic tachycardia is far slower than the AFCL during AF. However, breakthroughs related to localized ectopy may not be stationary, but wide-spread, do not show prematurity in coupling interval, and in most cases do show R-waves in the electrograms. Thus, identification of ectopic focal discharges during $\mathrm{AF}$ remains a challenge. 


\section{Introduction}

The mechanisms of initiation and perpetuation of atrial fibrillation (AF) are still unclear. A variety of re-entry mechanisms have been proposed to contribute to the perpetuation of $\mathrm{AF}^{1,2}$ including multiple wavelets, meandering or stationary re-entry circuits or rotors. ${ }^{3-7}$ While these models consider continuously conducted excitation as the source of local atrial activation, other experiments suggested that also ectopic discharges alone could contribute to AF perpetuation. ${ }^{8,9}$ Interestingly, epicardial mapping studies demonstrated that in patients with persistent AF the rate of activations showing radial spread of activation (breakthroughs) was 2-3 fold higher than in patients with acutely induced AF. ${ }^{2,10}$ However radial spread of activation from a localized site during AF does not allow direct conclusion regarding the mechanism of these activations. Breakthroughs might either be explained by transmural conduction of fibrillation waves or by ectopic focal discharges. ${ }^{2,11,12}$ Ectopic focal discharges might be caused by changes in atrial calcium-handling as observed AF patients and animal models for AF. ${ }^{13-15}$

Flecainide has been shown to open ryanodine receptors thereby reducing calciumspark mass. ${ }^{16}$ Therefore, flecainide might be effective to suppress ectopic focal discharges during AF.

In this study, we used a model of focal AF suggested by Scherf et al.. ${ }^{8}$ We applied aconitine on the left atrial epicardium in goats to induce stationary ectopic focal discharges. The objectives of this study were: 1 . to study the complex interaction between localized focal discharges and fibrillation waves during AF, 2. to identify criteria for detection of ectopic focal discharges during $\mathrm{AF}$, and 3 . to study the effect of flecainide on AF maintained by ectopic focal discharges. 


\section{Methods}

All experimental procedures were approved by the local ethical committee for animal experiments of Maastricht University.

\section{Goat Model: Open chest experiments}

In this study, 6 goats were anesthetized with sufentanyl $(6 \mathrm{mg} / \mathrm{kg} / \mathrm{h})$, midazolam $(0.8$ $\mathrm{mg} / \mathrm{kg} / \mathrm{h})$ and pancuronium $(0.3 \mathrm{mg} / \mathrm{kg} / \mathrm{h})$. After left-sided thoracotomy, a high-density endo-epicardial mapping device was positioned in the left atrium as described previously. ${ }^{10,11}$ The device was carefully closed to make contact with the endocardial bundle network and the epicardial surface. When satisfactory signals were present (sharp deflections in the absence of injury currents) the distance between the endocardial and the epicardial part of the device was fixated with a screw $(2.5-3.0$ $\mathrm{mm})$. A silver plate in the thoracic cavity served as indifferent electrode. Unipolar signals were recorded during AF and during atrial pacing using a custom-made 256channel mapping amplifier (filtering bandwidth 0.1-400 Hz, sampling rate $1 \mathrm{kHz}, \mathrm{A} / \mathrm{D}$ resolution 16 bits). A train of 10 basic stimuli (S1, pulse duration $1 \mathrm{~ms}$, BCL $200 \mathrm{~ms}$ ) at twice diastolic pacing threshold was followed by an extra-stimulus (S2) starting about 30 ms below the expected atrial effective refractory period (AERP) with a 2-ms increment. The shortest coupling interval able to elicit a propagated atrial response was taken as the AERP. AF was induced by atrial burst pacing.

After baseline measurements of refractory period, conduction velocity during atrial pacing and AF duration, aconitine $(0.5 \mathrm{mg})$ was applied at the centre of the mapping area of the same animal that had previously received burst pacing for AF induction. Epicardial aconitine application in the middle of the mapping area caused a regular and stable atrial tachycardia (CL: 200-230 ms) after 10min. 20-30min after aconitine application, AERP was determined in the middle of the mapping area and at two 
opposing sites outside the mapping area. Thereafter, AF was induced by atrial burst pacing.

\section{Analysis of fibrillation electrograms}

AF-electrogram files of 4 seconds were analyzed. Local activation times were identified as points of maximum negative $\mathrm{dV} / \mathrm{dt}$ in each unipolar electrogram. Median AF cycle length (AFCL) was calculated using all fibrillation intervals of all electrodes. Conduction times were determined by calculating the difference in activation time between each electrode and its neighbors.

Identification of individual fibrillation waves followed an algorithm described earlier. ${ }^{11,12}$ Waves were classified as:

a) Peripheral waves if their earliest activation time point was at the border of the mapping area or

b) Breakthrough if their earliest activation time point was within the mapping area.

The starting points of all breakthroughs were then tested for their plausibility to result from transmural conduction using a previously published algorithm. ${ }^{12}$ For this purpose the closest contralateral activation time points within an area of $5 \times 5$ electrodes $(8.0 \times 8.0 \mathrm{~mm})$ in the opposing layer of the atrial wall were determined. Breakthroughs were considered to be due to transmural conduction of existing fibrillation waves if the calculated conduction velocity between the contralateral source and the site of breakthrough was between $100-20 \mathrm{~cm} / \mathrm{s}$, questionable if it was between $20-5 \mathrm{~cm} / \mathrm{s}$ and not explainable by transmural conduction if no preceding activity within this given time range was found. ${ }^{11,12}$ Additionally, the presence of a unipolar R-wave (>1 mV) at the breakthrough sites was determined. Electrograms with an unstable baseline or with large superimposed ventricular far-field complexes 
were excluded. The degree of prematurity of "focal" fibrillation waves was determined by comparing their coupling interval with the average AF cycle length (AFCL) at the site of origin. ${ }^{17}$

\section{Quantification of endo-epicardial dissociation}

Electrical dissociation of between the epicardial layer and the endocardial bundle network was determined based on differences in activation time or direction of propagation of fibrillation waves between the two layers as described previously..$^{11,12}$ The endo-epicardial dissociation was expressed as percentage of the number and percentage of the surface area of dissociated and non-dissociated fibrillation waves.

\section{Flecainide experiments}

Flecainide $(0.2 \mathrm{mg} / \mathrm{kg} / \mathrm{min}$, i.v. $)$ was infused $60 \mathrm{~min}$ after aconitine-application. Endpoints of the cardioversion experiments were cardioversion to sinus rhythm, ventricular arrhythmias, QRS prolongation of $>100 \%$. Measurements of AFinducibility and AERP-measurement were repeated after cardionversion to sinus rhythm.

\section{Statistical analysis}

All data are expressed as mean \pm SEM. Significance of differences in means between baseline and after aconitine application was calculated using a one way ANOVA with Newman-Keuls post-test and a paired Student t-test for significance of differences in means between the endocardial and the epicardial layer in the same specimen. $\mathrm{P}$ values $<0.05$ were considered significant. 


\section{Results}

\section{Atrial tachycardia induced by epicardial application of aconitine}

Approximately 10min after topical application of aconitine in the middle of the mapping area a regular and stable atrial tachycardia (CL: 200-230 ms) occurred (Fig. 1). The earliest atrial activation time of each atrial tachycardia was localized in the middle of the epicardial mapping area at the site of aconitine application. The tachycardias were characterized by homogeneous, radial spread of activation. Electrograms at the site of earliest activation showed S-wave morphology. The earliest atrial activation time in the endocardial mapping area was registered with a transmural time delay of $6.6 \pm 0.2 \mathrm{~ms}$. However, epicardial aconitine-application did not induce AF in our model.

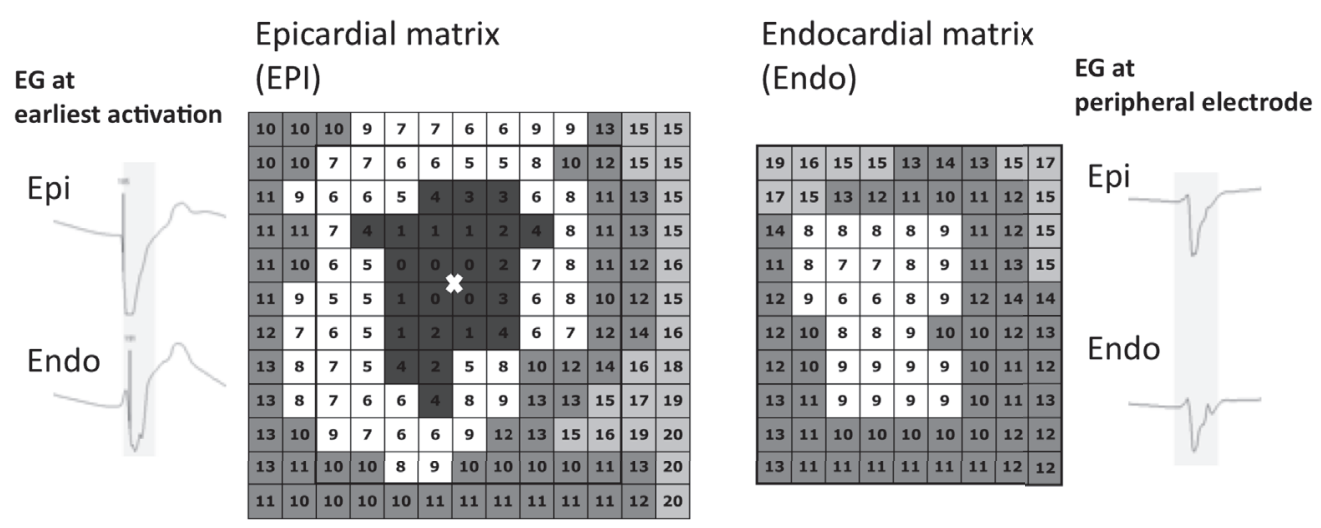

Figure 1: Isochronal map of atrial electrical activation pattern from simultaneous endo-epicardial recordings of aconitine-induced atrial tachycardia (time between isochrones $5 \mathrm{~ms}$ ). Point of earliest activation is on the epicardial plane in the middle of the mapping area at the site of aconitine application followed by a homogenious radial spread of activation. The earliest atrial activation time in the endocardial mapping area was registered with a transmural time delay of $6.6 \mathrm{~ms}$. 


\section{Effect of aconitine on refractory period and conduction velocity}

The effect of aconitine on refractoriness and conduction velocity is summarized in figure 2. Atrial regular pacing (BCL 200ms) from 2 different directions did not reveal any changes in atrial local conduction velocity by aconitine (Fig. 2A). Epicardial aconitine application did not change AERP at a BCL of $200 \mathrm{~ms}$, neither at the site of aconitine application nor in its surrounding in the left atrium (Fig. 2B).
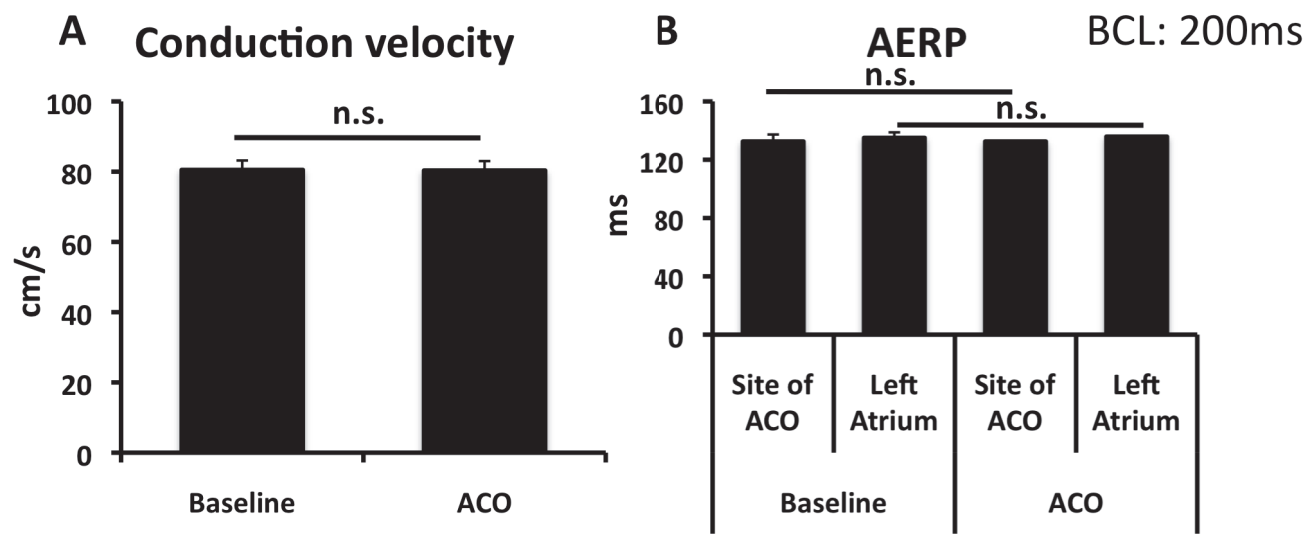

Figure 2: Effect of aconitine on the substrate for AF. (A) Atrial local conduction velocity (BCL: $200 \mathrm{~ms}$ ) before and after aconitine. (B) AERP (BCL: $200 \mathrm{~ms}$ ) at the site of aconitine application and in its surrounding in the left atrium. 


\section{AF before and after epicardial aconitine application}

Figure 3 shows the influence of aconitine on fibrillatory process during AF. Burstpacing induced AF-episodes after aconitine application were significantly longer compared to AF-episodes before aconitine application (Fig. 3A). AFCL was not changed by aconitine (Fig. 3B). The number and the surface area of fibrillation waves showing endo-epicardial dissociation was not altered significantly by aconitine (Fig. 3C). After aconitine application the rate of breakthroughs was 10-fold higher than before aconitine application (Fig. 3D).
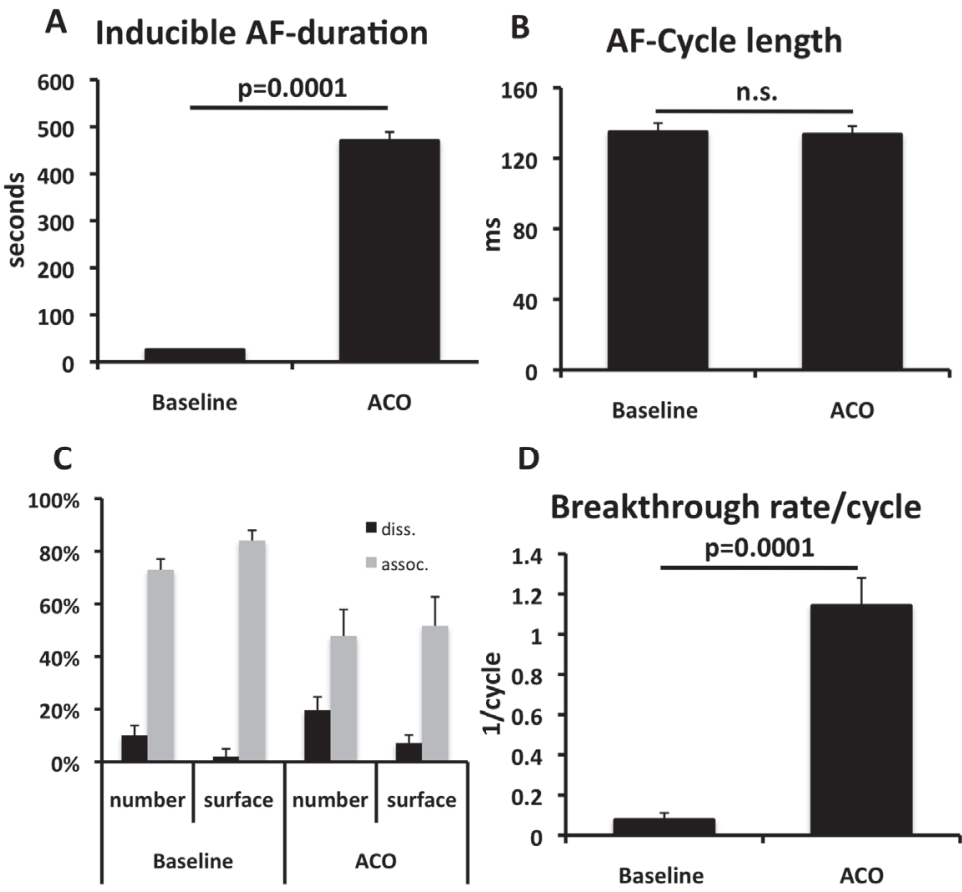

D

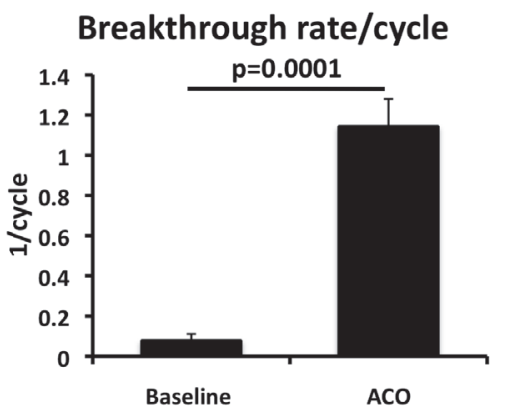

Figure 3: Influence of aconitine on fibrillatory process during AF: (A) Inducible AF-duration, (B) AF-cycle length and (C) number and the surface of dissociated or non-dissociated waves and (D) the breakthrough rate/cycle before and after aconitine are shown. 


\section{Characterization of breakthroughs}

While the site of earliest activation during the aconitine induced atrial tachycardia occurred exactly at the site of aconite application, after induction of AF the breakthroughs were spread around this site (mean distance from site of aconitine application: $4.7 \pm 1.1 \mathrm{~mm}$ ) (Fig. 4). The degree of prematurity breakthroughs was determined by comparing their coupling interval preceding the breakthroughs with the average $A F C L$ at the site of origin. Epicardial coupling intervals preceding the breakthroughs at the site of earliest activation did not differ significantly from the average AFCL at this site (Fig. 5A). Additionally, a high percentage (82\%) (Fig. 5B) of unipolar atrial electrograms at the site of earliest activation did show R-waves $(>1 \mathrm{mV})$. Representative EGs are shown in $3 \mathrm{C}$. However, based on the assumption of physiological endo-epicardial conduction velocities (between $100-20 \mathrm{~cm} / \mathrm{s}$ ) only $44 \%$ of all breakthroughs could be explained by transmural activation (breakthrough) from preceding contralateral waves. The remaining $56 \%$ could not be explained by transmural activation. 

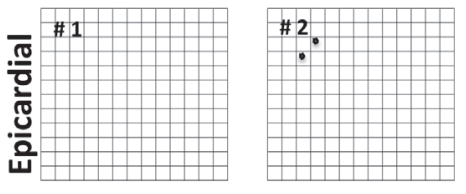

Breakthroughs during AF before ACO
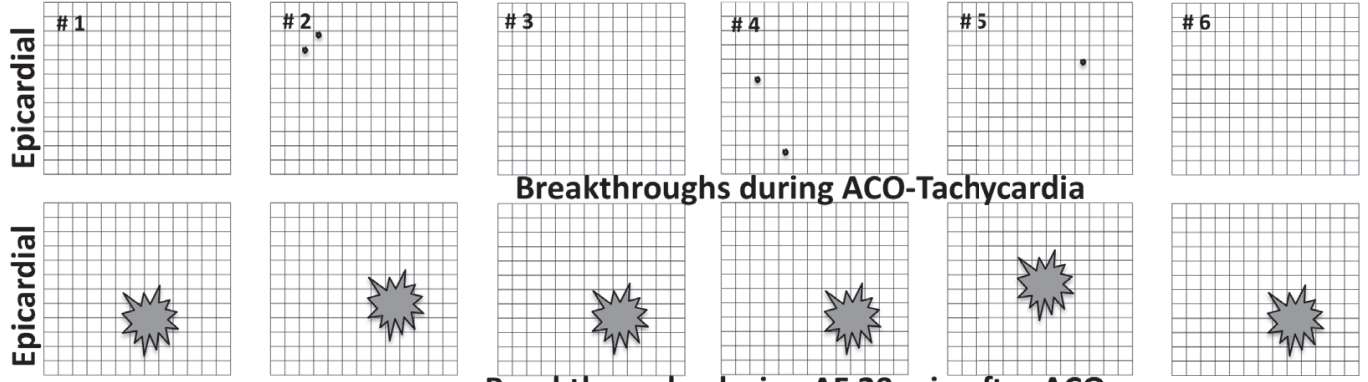

\section{Breakthroughs during ACO-Tachycardia}
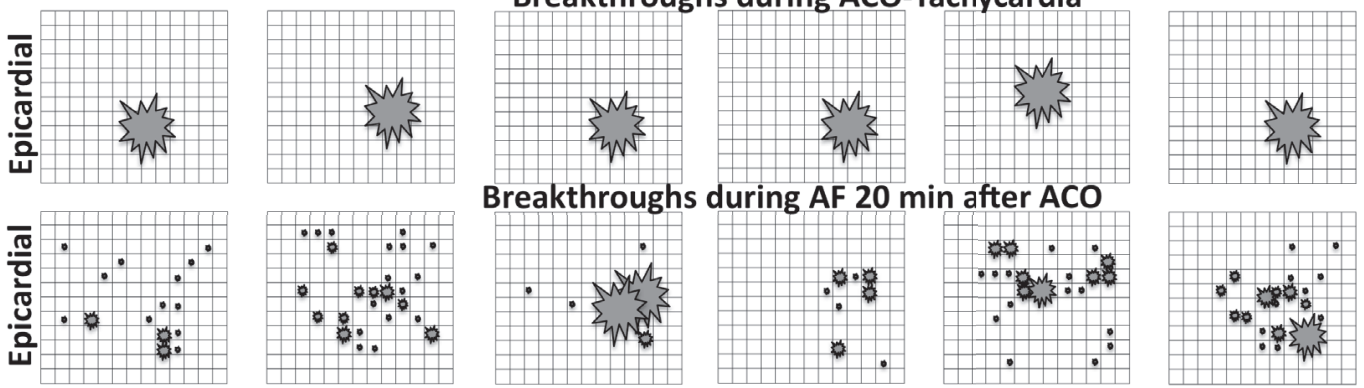

Figure 4: Local distribution of new appearing waves (breakthroughs, baseline (at the top), during atrial tachycardia induced by aconitine application in the middle of the mapping area (second row) and during AF after epicardial aconitine-

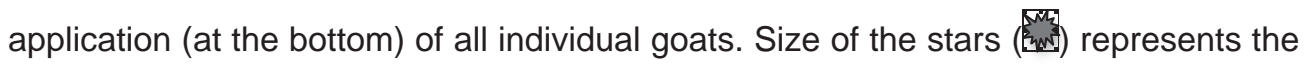
number of breakthroughs at one location.

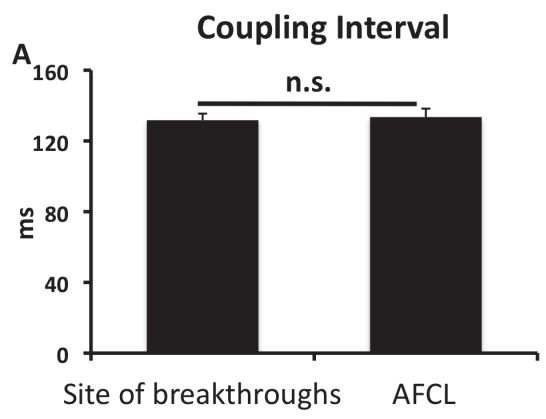

B EG at site of earliest activation

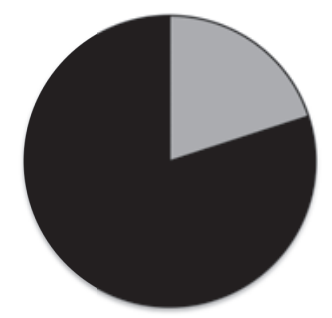

口no R-Wave

- R-Wave

Figure 5: (A) Epicardial coupling intervals of earliest activation at the site of breakthroughs compared to AFCL of the left atrium. (B) Percentage of unipolar atrial electrograms at the site of earliest activation with or without R-waves. (C) Representative electrograms at the site of earliest activation. 


\section{Effect of flecainide}

Flecainide completely inhibited the focal atrial tachycardia induced by aconitine and prolonged atrial cycle length (Fig. 6A). Burst-pacing induced AF-episodes after aconitine application were shortened from $470 \mathrm{~s}$ to $22 \mathrm{~s}$ after flecainide (Fig. 6B). AERP was prolonged (Fig. 6C) and local conduction velocity during regular pacing ( $\mathrm{BCL} 200 \mathrm{~ms}$ ) was reduced $(53 \mathrm{~cm} / \mathrm{s}$ vs. $79 \mathrm{~cm} / \mathrm{s}$ before flecainide, $p<0.01$ ) by flecainide.

Figure 6: Effect of flecainide:
(A) Atrial-cycle length,
(B) inducible AF-duration and
(C) AERP (BCL: $200 \mathrm{~ms})$

at baseline, after aconitine and after flecainide.
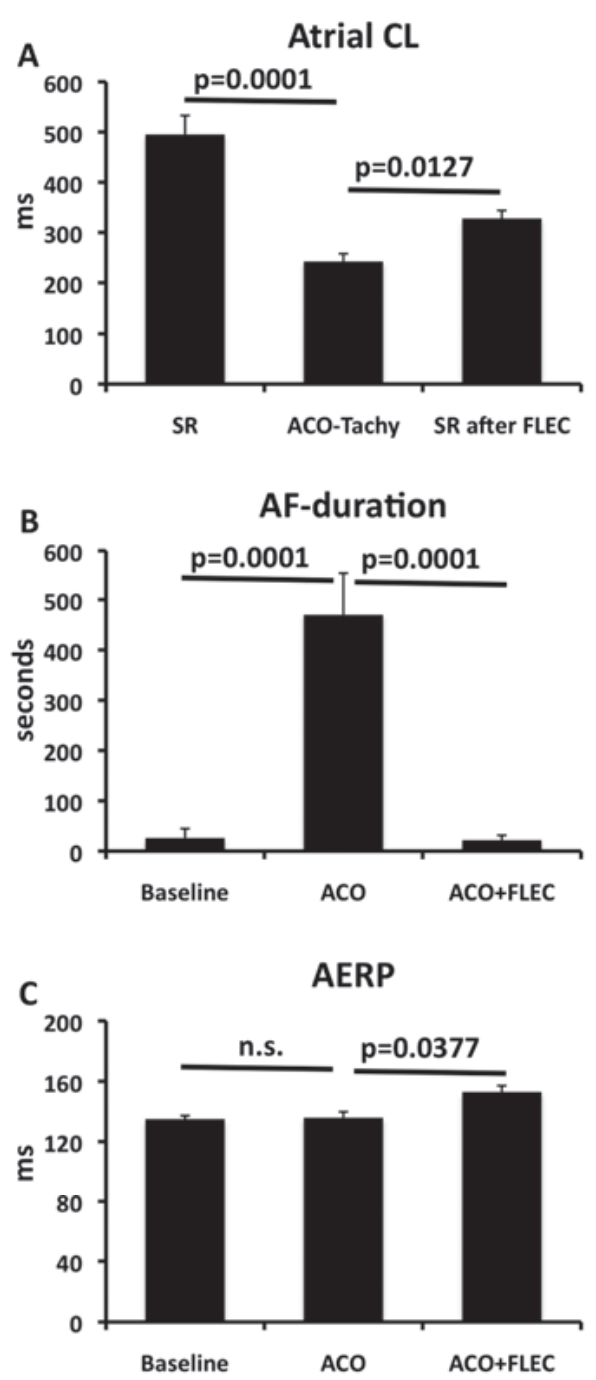


\section{Discussion}

Epicardial aconitine application elicited a regular and rapid atrial tachycardia originating from the site of aconitine application with a rapid radial spread of activation. Aconitine application did not influence AERP or local conduction velocity. However, despite the lack of changes in refractoriness and conduction velocity, the duration of AF-episodes induced by burst stimulation was longer and the number of breakthroughs increased by a factor 10 after aconitine application. The degree of endo-epicardial dissociation did not change after aconitine application. Breakthroughs were not stationary but wide-spread around the site of aconitine application, lacked prematurity, and most of them showed an R-wave morphology in the electrograms. Flecainide suppressed aconitine-induced atrial tachycardia and shortened the duration of $\mathrm{AF}$ episodes.

\section{Potential mechanisms of AF}

After decades of basic and clinical research, the mechanisms of initiation and perpetuation of atrial fibrillation are still not fully understood. ${ }^{1}$ Different types of reentry like multiple wavelets, rotors, or their combination have been described as perpetuators of $\mathrm{AF}^{3-7}$ In the 1990s, Haissaguerre et al. introduced the concept of "focal activity" as a trigger and perpetuator of paroxysmal forms of AF. ${ }^{18}$ In a group of 45 patients with AF they could demonstrate that the paroxysms were induced by focal activity (bursts) mainly originating from the myocardial sleeves in the pulmonary veins. The local nature of these depolarizations is suggestive for ectopic focal discharges in the pulmonary veins as the source of the fibrillatory process. Patterson et al. demonstrated that bursts from the pulmonary veins in patients often are initiated by atrial tachycardia followed by a pause. Endocardial mapping of the 
pulmonary veins revealed focal spread of activation of pulmonary vein firing. ${ }^{19}$ However, radial spread of activation from a localized site during AF does not allow direct determination of the mechanism of an arrhythmia. Therefore, in this study, we investigated the complex interaction between stationary ectopic focal discharges induced by aconitine and fibrillation waves. Furthermore, we tested the validity of traditionally accepted criteria to identify ectopic focal discharges during AF.

\section{Aconitine produces AF maintained by ectopic focal discharges}

The first in-vivo model of ectopic focal discharges triggering atrial tachyarrhythmias goes back to experiments of Scherf et al. In 1949, they applied aconitine to atria of dogs and induced thereby $\mathrm{AF}^{8}$ In this study, we used a modification of this model. Local epicardial application of aconitine on the left atrium of goats caused a rapid regular atrial tachycardia with a rapid radial spread of activation originating from the site of aconitine-application. Unipolar local electrograms at the site of earliest activation did show S-wave morphology demonstrating that ectopic focal discharges at this site generate this tachycardia. To investigate the effect of stationary localized ectopic focal discharges induced by aconitine on the fibrillatory process during AF, we induced AF by atrial burst pacing after aconitine application. AF-episodes were much longer than during the baseline measurement before aconitine application. Also, aconitine caused a strong increase in breakthrough rate during AF. Longer AF episodes after aconitine application might either be caused by disseminated ectopic discharges induced by aconitine or by changes in the substrate for AF e.g. by changes in refractoriness or atrial conduction. ${ }^{1,20-23}$ Additionally, increased endoepicardial dissociation during AF after aconitine application might increase the likelihood for transmural conduction, which could increase the number of breakthroughs. ${ }^{11,12}$ Endo-epicardial mapping studies revealed no changes in 
refractoriness, atrial conduction or endo-epicardial dissociation during $\mathrm{AF}$ after aconitine application suggesting an unchanged substrate for AF. To test for the plausibility of transmural conduction versus ectopic focal discharges as sources of breakthroughs, we used high-resolution simultaneous endo-epicardial in-vivo mapping. ${ }^{11,12}$ Only $44 \%$ of all breakthroughs could be explained by transmural conduction. Together with the fact that aconitine increased the rate of breakthrough by factor 10 by enhancing the propensity to ectopic focal discharges these data suggest that the far majority of breakthroughs during AF after aconitine application is caused by ectopic focal discharges. Furthermore, in this model the strong increase of AF stability is due to ectopic focal discharges induced by aconitine but not due to changes in refractoriness or conduction velocity.

\section{A single and low frequency site of ectopy is sufficient to increase AF stability}

A very remarkable finding in this study is that if only one site shows an enhanced propensity to ectopy AF duration may become much longer. This even holds true if as it is the case here - the cycle length of this ectopy is actually longer than the cycle length during AF. The presence of ectopic focal discharges during AF has indirectly been postulated by many studies on alterations of calcium $\left(\mathrm{Ca}^{2+}\right)$ handling during AF. ${ }^{1}$ Very recently we could directly demonstrate the existence of ectopic focal discharges during AF by high density simultaneous endo-epicardial mapping in goats with persistent AF. This study demonstrated that $13 \%$ of all breakthroughs are likely due to ectopic focal discharges (not more than $\sim 1.5 \%$ of all fibrillation waves). It remained unclear however what the quantitative contribution of ectopic focal discharges to the perpetuation of AF actually is. The present study demonstrates that a single site of ectopy might already be sufficient to very significantly prolong duration of AF episodes. 


\section{Traditional ectopy criteria fail to detect ectopic focal discharges during AF}

Subsequently, we studied whether the electrophysiological behavior of the breakthroughs observed in this study - largely caused by ectopic focal discharges would meet the traditional criteria for ectopic activity. ${ }^{17}$ We first tested for the presence of unipolar R-wave at the breakthrough sites. In the case of ectopic focal discharges, a unipolar electrogram recorded at the site of ectopy should show Swave morphology, whereas in the case of transmural conduction of pre-existing fibrillation waves an R-wave is expected. Secondly, the degree of prematurity of "focal" fibrillation waves was determined to identify a rapidly firing focus during AF. Finally, we tested local stationarity as a criterion, which would be particularly applicable in the case of this study because we knew where the site of maximal ectopic propensity was localized. To our surprise, breakthroughs related to localized ectopy induced by aconitine were not stationary, but wide-spread around the site of aconitine application. They did not show prematurity in coupling interval and the majority of them did show R-waves. This indicates that traditionally used parameters to identify ectopic focal discharges as a source of breakthroughs during AF failed to identify aconitine-induced "focal" breakthroughs during AF. Several factors might contribute to this unexpected finding. Ectopic discharges occurring at the surface of the atrium in the middle of the mapping area should generate electrograms with typical configuration for focal discharges (S-wave). This morphology was clearly visible in the sustained atrial tachycardia induced by aconitine before induction of AF. During AF, however, the gradient of ectopic propensity surrounding the crystal interacts with the heterogeneity in regaining excitability. This complex interaction of the sequence of recovery and the spatial gradient in ectopic propensity might have caused ectopic focal discharges occurring not just at the very site of aconitine application but also more remote from it. This includes the possibility that ectopic 
focal discharges may occur in deeper layers of the atrial wall so that the resulting fibrillation waves propagates a small distance before in encounters the surface of the atrium. This small distance might be sufficient to produce local source currents generating an R-wave. Finally, the fact that there is no prematurity of the activations leading to breakthroughs caused by ectopic focal discharges might be explained by the short excitable gap in this model and also by the fact that as soon as a fibrillation wave originated by a very early ectopic focal discharge starts to propagate the prematurity will get lost very quickly because of the low conduction velocity in an area with low excitability. Thus, our data imply that identification of an ectopic focal discharges during the fibrillatory process of AF based on electrogram morphology, stationarity, and prematurity might be less reliable than previously believed.

\section{Effect of flecainide on focal AF}

To further support the hypothesis that the occurrence of focal breakthroughs may contribute to the stabilization of AF after aconitine application, we investigated the effect of inhibition of aconitine-induced ectopic discharges on AF-duration. At the molecular level, aconitine binds to sodium channels and prolongs their open state favoring entry of a large quantity of sodium into the cytosol, which may result in $\mathrm{Ca}^{2+}$ overload and triggered activity. ${ }^{13,14,24-26}$ Sodium channel blockade has been shown to inhibit AF in the presence of aconitine. ${ }^{27}$ Additionally, the sodium blocker flecainide blocks open ryanodine receptors, increases the frequency of spontaneous $\mathrm{Ca}^{2+}$ release which reduces the $\mathrm{Ca}^{2+}$-spark mass. ${ }^{16}$ This reduced spark mass has been shown to lower the likelihood for proarrhythmic $\mathrm{Ca}^{2+}$ waves. In our study, flecainide completely inhibited the occurrence of spontaneous atrial tachycardia after aconitine and inducible AF-episodes were significantly shortened. This generally suggests an important role of focal discharges for increased breakthrough rate and longer AF- 
episodes after aconitine-application. However, in our study flecainide also elicited additional effects, like AERP-prolongation and conduction-velocity. Several different mechanisms have already been proposed to be responsible for the antiarrhythmic effect of flecainide. Wijffels et al. suggested that the antiarrhythmic effect of flecainide was due to a widening of the excitable gap during $A F .^{28}$ In the goat model of AF, they demonstrated that the 'wavelength' of fibrillation waves (atrial refractory period $x$ conduction velocity) was shortened by flecainide, which in itself would favor the persistence of AF. However, they found that cardioversion of AF by a variety of antiarrhythmic compounds (including flecainide) always went along with widening of the temporal excitable gap (difference between AF cycle length and atrial refractoriness during $\mathrm{AF}$ ) despite very different effects on refractory period and conduction velocity. A related mechanism was suggested by Kneller et al. in computer modeling studies. They demonstrated that because flecainide reduces conduction velocity preferentially at sites of wave turning fibrillation waves are more likely to collide with boundaries of the available substrate. ${ }^{29}$ In goats with 6 months of AF, Eckstein et al. showed that flecainide partially resynchronized electrical activity between the thin subepicardial layer and the endocardial bundle network, reduced transmural conduction thus the breakthrough rate. ${ }^{30}$ Inhibition of focal discharges by flecainide as described in our study might be an additional mechanism, which might contribute to the potent antiarrhythmic effect of flecainide.

\section{Summary and conclusion}

The existence of a single localized site with high propensity to ectopy induced by aconitine causes a strong increase in AF stability. While the breakthrough rate strongly increases other parameters of the $\mathrm{AF}$ substrate remain unchanged supporting the hypothesis that the breakthroughs are due to ectopic focal discharges 
and that they significantly contribute to the high stability of AF. Breakthroughs related to localized ectopy during AF may not be stationary, but wide-spread, may lack prematurity in coupling interval, and may show R-waves. This suggests a complex interaction between ectopy and re-entry during the fibrillatory process. Identification of ectopic focal discharges during $\mathrm{AF}$ remains a challenge.

\section{References}

1. Schotten U, Verheule S, Kirchhof P, Goette A. Pathophysiological mechanisms of atrial fibrillation: a translational appraisal. Physiol Rev. 2011; $91: 265-325$.

2. Eckstein J, Verheule S, de Groot NM, Allessie M, Schotten U. Mechanisms of perpetuation of atrial fibrillation in chronically dilated atria. Prog Biophys Mol Biol. 2008; 97:435-451.

3. Mines GR. On dynamic equilibrium in the heart. J Physiol. 1913; 46:349-383.

4. Moe GK, Abildskov JA. Atrial fibrillation as a self-sustaining arrhythmia independent of focal discharge. Am Heart J. 1959; 58:59-70.

5. Kumagai K, Khrestian C, Waldo AL. Simultaneous multisite mapping studies during induced atrial fibrillation in the sterile pericarditis model. Insights into the mechanism of its maintenance. Circulation. 1997; 95:511-521. 
6. Mandapati R, Skanes A, Chen J, Berenfeld O, Jalife J. Stable microreentrant sources as a mechanism of atrial fibrillation in the isolated sheep heart. Circulation. 2000; 101:194-199.

7. Jalife J, Berenfeld O, Mansour M. Mother rotors and fibrillatory conduction: a mechanism of atrial fibrillation. Cardiovasc Res. 2002; 54:204-216.

8. Scherf D, Terranova R. Mechanism of auricular flutter and fibrillation. Am J Physiol. 1949; 159:137-142.

9. Lee S, Sahadevan J, Khrestian CM, Durand DM, Waldo AL. High density mapping of atrial fibrillation during vagal nerve stimulation in the canine heart: restudying the moe hypothesis. J Cardiovasc Electrophysiol. 2013; 24:328335.

10. Allessie MA, de Groot NM, Houben RP, Schotten U, Boersma E, Smeets JL, Crijns HJ. Electropathological substrate of long-standing persistent atrial fibrillation in patients with structural heart disease: longitudinal dissociation. Circ Arrhythm Electrophysiol. 2010; 3:606-615.

11. Eckstein J, Maesen B, Linz D, Zeemering S, van Hunnik A, Verheule S, Allessie M, Schotten U. Time course and mechanisms of endo-epicardial electrical dissociation during atrial fibrillation in the goat. Cardiovasc Res. 2011; 89:816-824.

12. Eckstein J, Zeemering S, Linz D, Maesen B, Verheule S, van Hunnik A, Crijns $\mathrm{H}$, Allessie MA, Schotten U. Transmural Conduction is the Predominant Mechanism of Breakthrough during Atrial Fibrillation: Evidence from Simultaneous Endo-epicardial High Density Activation Mapping. Circ Arrhythm Electrophysiol. 2013:6:334-341. 
13. Vest JA, Wehrens XH, Reiken SR, Lehnart SE, Dobrev D, Chandra P, Danilo P, Ravens U, Rosen MR, Marks AR. Defective cardiac ryanodine receptor regulation during atrial fibrillation. Circulation. 2005; 111:2025-2032.

14. Hove-Madsen L, Llach A, Bayes-Genis A, Roura S, Rodriguez Font E, Aris A, Cinca J. Atrial fibrillation is associated with increased spontaneous calcium release from the sarcoplasmic reticulum in human atrial myocytes. Circulation. $2004 ; 110: 1358-1363$.

15. Neef S, Dybkova N, Sossalla S, Ort KR, Fluschnik N, Neumann K, Seipelt R, Schondube FA, Hasenfuss G, Maier LS. CaMKII-dependent diastolic SR Ca2+ leak and elevated diastolic $\mathrm{Ca} 2+$ levels in right atrial myocardium of patients with atrial fibrillation. Circ Res. 2010; 106:1134-1144.

16. Hilliard FA, Steele DS, Laver D, Yang Z, Le Marchand SJ, Chopra N, Piston DW, Huke S, Knollmann BC. Flecainide inhibits arrhythmogenic $\mathrm{Ca} 2+$ waves by open state block of ryanodine receptor $\mathrm{Ca} 2+$ release channels and reduction of Ca2+ spark mass. J Mol Cell Cardiol. 2010; 48:293-301.

17. de Groot NM, Houben RP, Smeets JL, Boersma E, Schotten U, Schalij MJ, Crijns $\mathrm{H}$, Allessie MA. Electropathological substrate of longstanding persistent atrial fibrillation in patients with structural heart disease: epicardial breakthrough. Circulation. 2010; 122:1674-1682.

18. Haissaguerre $M$, Jais $P$, Shah DC, Takahashi A, Hocini M, Quiniou G, Garrigue S, Le Mouroux A, Le Metayer P, Clementy J. Spontaneous initiation of atrial fibrillation by ectopic beats originating in the pulmonary veins. $\mathrm{N}$ Engl J Med. 1998; 339:659-666. 
19. Patterson E, Jackman WM, Beckman KJ, Lazzara R, Lockwood D, Scherlag BJ, Wu R, Po S. Spontaneous pulmonary vein firing in man: relationship to tachycardia-pause early afterdepolarizations and triggered arrhythmia in canine pulmonary veins in vitro. J Cardiovasc Electrophysiol. 2007; 18:10671075.

20. Li D, Fareh S, Leung TK, Nattel S. Promotion of atrial fibrillation by heart failure in dogs: atrial remodeling of a different sort. Circulation. 1999; 100:8795.

21. Verheule S, Wilson E, Everett T 4t, Shanbhag S, Golden C, Olgin J. Alterations in atrial electrophysiology and tissue structure in a canine model of chronic atrial dilatation due to mitral regurgitation. Circulation. 2003; 107:26152622.

22. Verheule S, Sato T, Everett T 4t, Engle SK, Otten D, Rubart-von der LoM, Nakajima HO, Nakajima H, Field LJ, Olgin JE. Increased vulnerability to atrial fibrillation in transgenic mice with selective atrial fibrosis caused by overexpression of TGF-beta1. Circ Res. 2004; 94:1458-1465.

23. Neuberger HR, Schotten U, Verheule S, Eijsbouts S, Blaauw Y, van Hunnik A, Allessie M. Development of a substrate of atrial fibrillation during chronic atrioventricular block in the goat. Circulation. 2005; 111:30-37.

24. Sawanobori T, Hirano Y, Hiraoka M. Aconitine-induced delayed afterdepolarization in frog atrium and guinea pig papillary muscles in the presence of low concentrations of Ca2+. Jpn J Physiol. 1987; 37:59-79. 
25. Amran MS, Hashimoto K, Homma N. Effects of sodium-calcium exchange inhibitors, KB-R7943 and SEA0400, on aconitine-induced arrhythmias in guinea pigs in vivo, in vitro, and in computer simulation studies. J Pharmacol Exp Ther. 2004; 310:83-89.

26. Fu M, Li RX, Fan L, He GW, Thornburg KL, Wang Z. Sarcoplasmic reticulum $\mathrm{Ca} 2+$ release channel ryanodine receptor (RyR2) plays a crucial role in aconitine-induced arrhythmias. Biochem Pharmacol. 2008; 75:2147-2156.

27. Hashimoto N, Yamashita T, Fujikura N, Tsuruzoe N. NIP-141, a multiple ion channel blocker, terminates aconitine-induced atrial fibrillation and prevents the rapid pacing-induced atrial effective refractory period shortening in dogs. Europace. 2007; 9:246-251.

28. Wijfels MC, Dorland R, Mast F, Allessie MA. Widening of the excitable gap during pharmacological cardioversion of atrial fibrillation in the goat: effects of cibenzoline, hydroquinidine, flecainide, and d-sotalol. Circulation. 2000; 102:260-267.

29. Kneller J, Kalifa J, Zou R, Zaitsev AV, Warren M, Berenfeld O, Vigmond EJ, Leon LJ, Nattel S, Jalife J. Mechanisms of atrial fibrillation termination by pure sodium channel blockade in an ionically-realistic mathematical model. Circ Res. 2005; 96:e35-e47.

30. Eckstein J. The Three-dimensional Substarte of Atrial Fibrillation in the Goat. A Simultaneous Endo-Epicardial Mapping Study. Maastricht: Fysiology, Maastricht University; 2012. 
Chapter 7:

General Discussion 


\section{General Discussion}

Understanding the mechanisms involved in the initiation and progression of AF is important to optimize treatment of AF. The present study represents an integrative approach to assess arrhythmogenic mechanisms for the development and progression of AF under different pathophysiological conditions. The focus of this study lies on the characterization of the substrate for $A F$ in diabetes and obstructive sleep apnea, both associated with increased recurrences of AF and failure of pharmacological and catheter based ablation therapy. Subsequently, targeted intervention of newly identified pathophysiological mechanisms was tested on the induction and progression of AF. Importantly, atrial arrhythmogenic substrate is not just characterized by structural changes and alterations in atrial refractoriness but also by ectopic focal discharges inducing and perpetuating AF. Up to very recently, clear parameters to reliably identify ectopic discharges during AF were lacking complicating the development of targeted intervention. High-density endo-epicardial mapping was deployed to characterize the complex interaction between ectopic focal discharges and reentry mechanisms during $\mathrm{AF}^{1}$ but this technique has not been developed so far for clinical routine practice.

\section{Development of an atrial substrate for $\mathrm{AF}$ in diabetes}

Diabetes is a strong independent risk factor for AF and stroke..$^{2-4}$ Impaired LAemptying function might underlie reduced exercise tolerance and enhanced risk for strokes in diabetic patients. ${ }^{5-7}$ The mechanisms leading to the development of an arrhythmogenic atrial substrate and impaired LA-emptying function in diabetes are unknown. 
In this thesis, long-term diabetic conditions lead to a structural arrhythmogenic remodeling associated with local conduction disturbances and impairment of atrial emptying function. The activity of the protease CathepsinA was increased in atrial tissue. CathepsinA has been shown to be upregulated in the heart under several pathophysiological conditions and peptide hormones like angiotensin I and bradykinin have been described as substrates for CathepsinA in vitro. ${ }^{8,9}$ Inhibition of atrial CathepsinA-activity by a new CathepsinA inhibitor ${ }^{8}$ attenuated the development of arrhythmogenic substrate in diabetic rats. CathepsinA inhibition was associated with a reduction of left atrial bradykinin consumption, which may explain its antiremodeling effect. Pharmacological CathepsinA-inhibition might provide a new promising atrial up-steam therapy strategy in diabetes (Chapter 2).

\section{Arrhythmogenic mechanisms involved in atrial arrhythmogenisis in obstructive sleep apnea}

Patients with obstructive sleep apnea (OSA) show a high prevalence of atrial fibrillation (AF) ranging from 32 to $49 \%$ and patients with severe OSA have a 4 -fold increased risk for $\mathrm{AF}^{10-12} \mathrm{OSA}$ was the strongest predictor of recurrent $\mathrm{AF}$ following catheter ablation ${ }^{13}$ and patients with untreated OSA have a higher risk of recurrence of AF after successful cardioversion. ${ }^{14}$ Additionally, OSA is associated with failure of pharmacological antiarrhythmic treatment. ${ }^{15}$ The finding that appropriate treatment of OSA with continuous positive airway pressure (CPAP) is associated with lower

recurrence of $\mathrm{AF}$ suggests a causal association of OSA inducing AF ${ }^{16}$ However, the mechanisms causing AF in OSA are not completely understood. Obstructive apneas are caused by collapse of the upper airway during sleep resulting in repetitive forced inspiration against the obstructed upper airway. Correspondingly substantial negative changes in intratracheal pressure down to -100 mbar were observed in OSA 
patients. ${ }^{12}$ Besides, obstructive respiratory events are associated with hypoxia and hypercapnia.

In this thesis, applied negative tracheal pressure (NTP) during tracheal obstruction in obstructive sleep apnea was identified as the main contributor to arrhythmogenic electrophysiological changes during obstructive respiratory events. NTP led to pronounced shortening of atrial effective refractory. This was mediated by increased vagal activation as indicated by complete prevention by atropin and vagotomy. By contrast, hypoxia and hypercapnia without applied NTP did not result in shortening of atrial refractoriness (Chapter 3). Selective blockade of single atrial potassium channels by antiarrhythmic drugs currently used in the clinic (sotalol, amiodarone) failed to inhibit the arrhythmogenic electrophysiological changes. Just the combined blockade of several potassium channels resulted in a sufficient antiarrhythmic effect, supporting the need for multichannel blockers (Chapter 4). This suggests that obstructive respiratory events create a highly arrhythmogenic substrate in the atrium that is difficult to suppress using available AADs and differs significantly from other conditions for which AADs have shown sufficient efficacy. ${ }^{17,18}$ This is in line with a resent clinical study, which showed that patients with severe OSA respond less likely to antiarrhythmic drug therapy for AF than those with milder forms of OSA. Interestingly, non-responders to ADDs had higher apnea-hypopnea indexes than responders. Minimum oxygen saturation did not differ between the two groups. ${ }^{15}$ In line with our study, this study suggests that not hypoxia but the obstructive events seem to be important for AF recurrence, limiting AAD efficacy in patients with OSA. ${ }^{18}$ In our pig model for obstructive sleep apnea, we showed antiarrhythmic effects of modulation of sympathetic system by renal sympathetic denervation (Chapter 5). Interestingly, renal sympathetic denervation displayed a substantially more 
pronounced antiarrhythmic effect in this pig model for sleep apnea compared to clinically used antiarrhythmic drugs like sotalol or amiodarone. Renal denervation might represent a promising antiarrhythmic treatment strategy, particularly in patients with obstructive sleep apnea, which has been shown to be associated with failure of pharmacological antiarrhythmic treatment. ${ }^{15}$

In a small study in humans, the atrial antiarrhythmic effects of RDN combined with circumferential pulmonary vein isolation was investigated. ${ }^{19}$ Patients who received both procedures showed significant reductions in average systolic and diastolic blood pressure, whereas those in the circumferential pulmonary vein isolation-only group did not show any significant improvement in blood pressure. Moreover, at one-year follow-up, $69 \%$ of patients who received both procedures no longer had AF, compared to only $29 \%$ of those in the circumferential pulmonary vein isolation-only group. In a case report, even RDN without circumferential pulmonary vein isolation reduced blood pressure and attenuated paroxysmal AF-episodes, which were symptomatic and drug-resistant before $\mathrm{RDN}{ }^{20}$ Whether renal denervation can also display antiarrhythmic effects in normotensive patients in the clinical setting needs to be investigated in future studies.

\section{Identification of ectopic discharges during AF}

Besides structural changes and alterations in atrial refractoriness, also ectopic focal discharges are discussed to play a relevant role for the induction and perpetuation of AF. Epicardial mapping studies in patients with acute and persistent AF revealed a 2-3 fold increase in wavefronts with radial spread of activation appearing in the middle of the mapping area, which cannot be explained by propagation in the epicardial plane. ${ }^{21}$ Origin of these waves could either be ectopic focal discharges triggerd by delayed after-depolarisations or waves originating from the opposing layer 
of the atrial wall encountering excitable tissue at the site of breakthrough. ${ }^{1,22-23}$ Simultaneous epi-endocardial mapping of atrial activation in goats revealed that the majority of breakthrough events could be explained by transmural conduction from the contralateral wall. ${ }^{1,23}$ Ectopic discharges might be caused by changes in atrial calcium-handling as observed in both, AF-patients and AF animal models. ${ }^{24-26}$ However, simultaneous endo-epicardial high density mapping can at the present time not be implemented in clinical routine of $\mathrm{AF}$ ablation. Although parameters to reliably identify ectopic discharges during AF are highly desirable they are currently not available. Also, the precise interaction between ectopic focal discharges and reentry mechanisms in AF are unknown.

In this thesis, ectopic focal discharges were induced by epicardial application of aconitine, which increases the propensity to focal discharges (chapter 6). Subsequently, the interaction between $\mathrm{AF}$ and ectopic focal discharges was characterized. Epicardial application of aconitine resulted in focal discharges not strictly limited to the site of aconitine application. AF-episodes after aconitine application lasted longer and were associated with increased occurrence of breakthroughs independent of changes in atrial refractoriness and atrial conduction. Simultaneous epi-endocardial mapping revealed that just a minority of breakthroughs could be explained by transmural conduction. Therefore the majority of breakthroughs must have been caused by ectopic focal discharges induced by aconitine. However, traditionally used parameters to characterize focal discharges during AF failed to identify breakthroughs after aconitine application, suggesting a complex interaction between focal discharges and the fibrillatory process. Hove Madsen reported increased incidences of spontaneous calcium release events ("sparks") in patients with AF. ${ }^{25}$ Sparks can trigger intracellular calcium waves, which then can trigger delayed after-depolarisations and ectopic focal discharges. 
Knollmann reported on the effect of flecainide on sparks and could demonstrate that flecainide blocks ryonadine receptor calcium release channels in the open state and thereby reduces the spark mass. ${ }^{27,28}$ In our study, flecainide suppressed focal discharges induced by aconitine and reduced AF-duration supporting the concept that focal discharges play an important role in maintaining AF and that its inhibition (as shown by flecainide) results in an antiarrhythmic effect. Valid criteria to identify focal discharges during AF would largely facilitate the development of new treatment strategies targeting focal discharges (Chapter 6). Unfortunately, our study demonstrates that traditional criteria for ectopy are insufficient to identify focal discharges during AF.

\section{Conclusions}

The arrhythmogenic substrate differs significantly dependent on the underlying pathophysiological conditions. Diabetes and OSA are both associated with an arrhythmogenic substrate for AF, which differs from other conditions like lone-AF, congestive heart failure or hypertension. As treatment of AF should depend on the underlying arrhythmogenic mechanisms, we tested the antiarrhythmic effects of interventions targeting the proposed mechanisms.

\section{As a result of this thesis, we conclude the following:}

1. Inhibition of the protease CathepsinA, which was highly upregulated in the atrium of diabetic rats attenuated the development of an arrhythmogenic atrial substrate. This suggests that CathepsinA inhibition might represent a promising target for antiarrhythmic upstream treatment in diabetes. 
2. Pronounced shortening in atrial refractoriness during obstructive respiratory events was associated with increased AF-inducibility in a pig model for obstructive sleep apnea. Importantly, antiarrhythmic drugs currently used in the clinic failed to inhibit the arrhythmogenic electrophysiological changes. Modification of the sympatovagal system by renal sympathetic denervation showed potent antiarrhythmic effects and provides a promising strategy to treat vagally mediated AF in OSA.

3. In a goat model for AF maintained by aconitine, we demonstrated a complex interaction between focal discharges and the fibrillatory process. Identification of focal discharges during $\mathrm{AF}$ is challenging, as traditional parameters failed to clearly discriminate between focal discharges and transmural conduction as the source of breakthroughs. Reduction of focal discharges by flecainide showed pronounced antiarrhythmic effects.

\section{References}

1. Eckstein J, Zeemering S, Linz D, Maesen B, Verheule S, van Hunnik A, Crijns $H$, Allessie MA, Schotten U. Transmural Conduction is the Predominant Mechanism of Breakthrough during Atrial Fibrillation: Evidence from Simultaneous Endo-epicardial High Density Activation Mapping. Circ Arrhythm Electrophysiol. 2013:6:334-341.

2. Kannel WB, Wolf PA, Benjamin EJ, Levy D. Prevalence, incidence, prognosis, and predisposing conditions for atrial fibrillation: population-based estimates. Am J Cardiol. 1998;82:2N-9N.

3. Brand FN, Abbott RD, Kannel WB, Wolf PA. Characteristics and prognosis of lone atrial fibrillation. 30-year follow-up in the Framingham Study. JAMA. 1985;254:3449-3453. 
4. Movahed MR, Hashemzadeh M, Jamal MM. Diabetes mellitus is a strong, independent risk for atrial fibrillation and flutter in addition to other cardiovascular disease. Int J Cardiol. 2005;105:315-318.

5. Regensteiner JG, Sippel J, McFarling ET, Wolfel EE, Hiatt WR. Effects of noninsulin-dependent diabetes on oxygen consumption during treadmill exercise. Med Sci Sports Exerc. 1995;27:875-881.

6. Regensteiner JG, Bauer TA, Reusch JE, Brandenburg SL, Sippel JM, Vogelsong AM, Smith S, Wolfel EE, Eckel RH, Hiatt WR. Abnormal oxygen uptake kinetic responses in women with type II diabetes mellitus. J Appl Physiol. 1998;85:310-317.

7. Wei M, Gibbons LW, Mitchell TL, Kampert JB, Lee CD, Blair SN. The association between cardiorespiratory fitness and impaired fasting glucose and type 2 diabetes mellitus in men. Ann Intern Med. 1999;130:89-96.

8. Ruf S, Buning C, Schreuder H, Horstick G, Linz W, Olpp T, Pernerstorfer J, Hiss K, Kroll K, Kannt A, Kohlmann M, Linz D, Hübschle T, Rütten H, Wirth K, Schmidt T, Sadowski T. Novel B-amino acid derivatives as inhibitors of cathepsin A. J Med Chem. 2012;55:7636-7649.

9. Ruf S, Buning C, Schreuder H, Linz W, Hübschle T, Linz D, Ruetten H, Wirth $\mathrm{K}$, Sadowski T. Inhibition of CatA: an emerging strategy for the treatment of heart failure. Future Medicinal Chemistry, 2013;5:399-409.

10. Johnson KG, Johnson DC: Obstructive sleep apnea is a risk factor for stroke and atrial fibrillation. Chest 2010; 138:239.

11. Gami AS, Pressman G, Caples SM, et al.: Association of atrial fibrillation and obstructive sleep apnea. Circulation 2004; 110:364-367.

12. Kasai T, Bradley TD: Obstructive sleep apnea and heart failure: pathophysiologic and therapeutic implications. J Am Coll Cardiol 2011; 57:119- 
127.

13. Matiello M, Nadal M, Tamborero D, et al.: Low efficacy of atrial fibrillation ablation in severe obstructive sleep apnea patients. Europace 2010; 2:10841089.

14. Kanagala R, Murali NS, Friedman PA, et al.: Obstructive sleep apnea and the recurrence of atrial fibrillation. Circulation 2003; 107:2589-2594.

15. Monahan K, Brewster J, Wang L, Parvez B, Goyal S, Roden DM, Darbar D. Relation of the severity of obstructive sleep apnea in response to antiarrhythmic drugs in patients with atrial fibrillation or atrial flutter. Am J Cardiol. 2012 Aug 1;110(3):369-72.

16. Kanagala R, Murali NS, Friedman PA, et al.: Obstructive sleep apnea and the recurrence of atrial fibrillation. Circulation 2003; 107:2589-2594.

17. Ehrlich JR, Biliczki P, Hohnloser SH, Nattel S. Atrial-selective approaches for the treatment of atrial fibrillation. J Am Coll Cardiol. 2008;51:787-792.

18. Linz D. Atrial fibrillation in obstructive sleep apnea: atrial arrhythmogenic substrate of a different sort. American Journal of Cardiology, 2012;110:1071.

19. Pokushalov E, Romanov A, Corbucci G, Artyomenko S, Baranova V, Turov A, Shirokova N, Karaskov A, Mittal S, Steinberg JS. A randomized comparison of pulmonary vein isolation with versus without concomitant renal artery denervation in patients with refractory symptomatic atrial fibrillation and resistant hypertension. J Am Coll Cardiol. 2012; 60:1163-1170.

20. Vollmann D, Sossalla S, Schroeter MR, Zabel M. Renal artery ablation instead of pulmonary vein ablation in a hypertensive patient with symptomatic, drugresistant, persistent atrial fibrillation. Clin Res Cardiol. 2012 [Epub ahead of print]

21. de Groot NM, Houben RP, Smeets JL, Boersma E, Schotten U, Schalij MJ, 
Crijns $\mathrm{H}$, Allessie MA. Electropathological substrate of longstanding persistent atrial fibrillation in patients with structural heart disease: epicardial breakthrough. Circulation. 2010;122:1674-1682.

22. Eckstein J, Verheule S, de Groot NM, Allessie M, Schotten U. Mechanisms of perpetuation of atrial fibrillation in chronically dilated atria. Prog Biophys Mol Biol. 2008; 97:435-451.

23. Eckstein J, Maesen B, Linz D, Zeemering S, van Hunnik A, Verheule S, Allessie M, Schotten U. Time course and mechanisms of endo-epicardial electrical dissociation during atrial fibrillation in the goat. Cardiovasc Res. 2011;89:816-824.

24. Vest JA, Wehrens XH, Reiken SR, Lehnart SE, Dobrev D, Chandra P, Danilo P, Ravens U, Rosen MR, Marks AR. Defective cardiac ryanodine receptor regulation during atrial fibrillation. Circulation. 2005; 111:2025-2032.

25. Hove-Madsen L, Llach A, Bayes-Genis A, Roura S, Rodriguez Font E, Aris A, Cinca J. Atrial fibrillation is associated with increased spontaneous calcium release from the sarcoplasmic reticulum in human atrial myocytes. Circulation. 2004; 110:1358-1363.

26. Neef S, Dybkova N, Sossalla S, Ort KR, Fluschnik N, Neumann K, Seipelt R, Schondube FA, Hasenfuss G, Maier LS. CaMKII-dependent diastolic SR Ca2+ leak and elevated diastolic $\mathrm{Ca} 2+$ levels in right atrial myocardium of patients with atrial fibrillation. Circ Res. 2010; 106:1134-1144.

27. Hilliard FA, Steele DS, Laver D, Yang Z, Le Marchand SJ, Chopra N, Piston DW, Huke S, Knollmann BC. Flecainide inhibits arrhythmogenic Ca2+ waves by open state block of ryanodine receptor $\mathrm{Ca} 2+$ release channels and reduction of Ca2+ spark mass. J Mol Cell Cardiol. 2010; 48:293-301. 
28. Watanabe H, Chopra N, Laver D, Hwang HS, Davies SS, Roach DE, Duff HJ, Roden DM, Wilde AA, Knollmann BC. Flecainide prevents catecholaminergic polymorphic ventricular tachycardia in mice and humans. Nat Med. 2009; 15:380-383. 


\section{Summary}

Diabetes and obstructive sleep apnea are associated with increased recurrence of atrial fibrillation (AF) and failure of pharmacological and catheter based ablation therapy. The present study was conducted to characterize the atrial substrate for AF in animal models for diabetes and obstructive sleep apnea and to identify new potential antiarrhythmic therapy strategies.

In Zucker diabetic fatty rats, a rat model for type 2 diabetes, we identified the development of an arrhythmogenic substrate mainly characterized by structural alterations leading to increased AF-susceptibility and impaired atrial emptying function. In atrial tissue, the enzyme CathepsinA, involved in angiotensin and bradykinin metabolism, was highly upregulated. Pharmacological inhibition of CathepsinA by SAR1 attenuated the development of a functional and arrhythmogenic atrial substrate representing a promising target for atrial upstream therapy in diabetes (Chapter 2).

To identify mechanisms involved in the increased AF-susceptibility in obstructive sleep apnea, we developed a pig model for OSA. In anesthetized pigs, applied negative tracheal pressure mimicking obstructive respiratory events lead to pronounced shortening of atrial effective refractory mediated by increased vagal activation (Chapter 3). Selective blockade of single atrial potassium channels by antiarrhythmic drugs currently used in the clinic (sotalol, amiodarone) failed to inhibit the arrhythmogenic electrophysiological changes. Just the combined blockade of several potassium channels resulted in a sufficient antiarrhythmic effect, supporting 
the need for multichannel blockers (Chapter 4). Interestingly, also modulation of autonomic nervous system by renal sympathetic denervation reduced OSA associated shortening in refractoriness. Therefore, renal sympathetic denervation might provide a promising tool to treat AF in obstructive sleep apnea (Chapter 5).

The interaction between ectopy and reentry mechanisms during AF is unclear. In a goat model, the existence of a single localized site with high propensity to ectopy induced by aconitine caused a strong increase in AF stability. While the breakthrough rate strongly increases other parameters of the AF substrate remain unchanged supporting the hypothesis that the breakthroughs are due to ectopic focal discharges and that they significantly contribute to the high stability of AF. However, breakthroughs related to localized ectopy induced by aconitine were not stationary, but wide-spread around the site of aconitine application. This suggests a complex interaction between ectopy and re-entry during the fibrillatory process. Identification of ectopic focal discharges during AF remains a challenge. New and valid mapping criteria are necessary to identify focal discharges during AF, which may be important for the development of new treatment strategies targeting focal discharges (Chapter $6)$. 


\section{Samenvatting}

Diabetes en het obstructief slaapapneusyndroom worden beide geassocieerd met een toename in het heroptreden van atriumfibrilleren (AF) en met het falen van medicamenteuze behandeling en catheterablatie. De huidige studie werd uitgevoerd om het atriale substraat van AF te karakteriseren in diermodellen van diabetes en van het obstructief slaapapneusyndroom, en om nieuwe potentiële anti-aritmische therapieën te identificeren.

In Zucker diabetische ratten, een diermodel voor type 2 diabetes, hebben we de ontwikkeling van een aritmogeen substraat bestudeerd. Deze ontwikkeling werd voornamelijk gekarakteriseerd door structurele veranderingen die leidden tot een verhoogde gevoeligheid voor $\mathrm{AF}$ en tot een verminderde atriale lediging (pompfunctie). In atriaal weefsel was de functie van het enzyme CathepsinA, dat deel uitmaakt van het angiotensine en bradykinine metabolisme, sterk opgewaardeerd. Medicamenteuze inhibitie van CathepsinA door SAR1 versterkte de ontwikkeling van een functioneel en aritmogeen atriaal substraat. Als zodanig vertegenwoordigt CathepsinA een veelbelovend aangrijppunt voor atriale 'upstream therapy' in de behandeling van diabetes (Hoofdstuk 2).

Om de mechanismes vast te leggen die betrokken zijn in de toegenomen gevoeligheid voor AF in het obstructief slaapapneusyndroom hebben we een varkensmodel voor obstructief slaapapneu ontwikkeld. In verdoofde varkens werd negatieve tracheale druk toegepast om de obstructieve ademhalingsepisodes na te bootsen. Dit leidde tot een uitgesproken verkorting van de atriale effectieve refractaire periode, door een toename in vagale activatie (Hoofdstuk 3). Het selectief blokkeren van geïsoleerde atriale kaliumkanalen door anti-aritmische medicijnen die gebruikt worden in de huidige klinische praktijk (zoals sotalol en amiodarone) was 
niet in staat om de aritmogene elektrofysiologische veranderingen te inhiberen. Enkel het simultaan blokkeren van meerdere kaliumkanalen leidde tot een afdoende antiaritmisch effect. Dit feit onderstreept de nood aan multikanaal blokkers (Hoofdstuk 4). Een interessante bevinding was dat ook het moduleren van het autonome zenuwstelsel door renale denervatie de verkorting van de refractaire periode, geassocieerd met obstructieve slaapapneu, bewerkstelligde. Hierdoor zou renale sympathische denervatie ook een veelbelovende therapie kunnen zijn om AF te behandelen in patiënten met obstructief slaapapneu (Hoofdstuk 5).

De interactie tussen ectopie- en 'reentry'-mechanismes gedurende AF is nog niet uitgeklaard. In een geit-model leidde de aanwezigheid van een enkele site met een hoge neiging tot aconitine-geïnduceerde ectopie tot een sterke toename in AFstabiliteit. Tevens observeerden we een sterke toename in het aantal 'breakthroughs' terwijl de overige parameters van het AF-substraat onveranderd bleven. Dit ondersteunt de hypothese dat het optreden van 'breakthroughs' een gevolg is van ectopische focale elektrische ontladingen en dat deze 'breakthroughs' gevoelig bijdragen tot de hoge stabiliteit van AF. Echter, 'breakthroughs' gerelateerd aan de aconitine-geïnduceerde plaatselijke ectopie waren niet stationair, maar traden op in een wijdverspreid gebied rondom de applicatieplaats van aconitine. Deze vaststelling veronderstelt een complexe interactie tussen ectopie en 'reentry' tijdens het fibrillatieproces. Het vastleggen van ectopische focale elektrische ontladingen blijt een uitdaging. Nieuwe en gevalideerde mappingcriteria zijn noodzakelijk om ectopische focale elektrische ontladingen tijdens AF te identificiëren. Deze nieuwe mappingcriteria zijn mogelijk een belangrijk onderdeel voor de ontwikkeling van nieuwe behandelingsstrategieën die focale elektrische ontladingen aanpakken (Hoofdstuk 6). 


\section{Acknowledgements / Nawoord}

My adventure in Maastricht started in 2009. During this time I worked in the lab of Klaus Wirth in Frankfurt and prepared my medical doctor thesis. I still remember when I met Uli the first time. He gave a talk about his work on atrial contractility and atrial function in Frankfurt. Although I already knew most of his research from his publications, I directly noticed that a very relevant part of Uli's work cannot be transferred in a written manuscript: His motivation to explain and convince other people of his ideas and his enthusiasm to question and test all own data before trusting them. I still think that this is the most relevant thing, which I learned during my time in Maastricht. Never trust your own data before questioning and reanalyzing them at least 2-4 times. This scepsis is the secret of getting reproducible and high quality data.

Uli, thank you for giving me the opportunity to become a member of your group, for your continuous support of my work during my time in Maastricht and also afterwards. I am happy about the collaborations, which are lasting until today. I enjoy working together with you.

CARIM is indeed a nice place to work: it profits from several things: the nice city Maastricht, and the extensive local, national and international networks and collaborations. However, the most important thing is the atmosphere in the group and the colleagues: Sander, Stef, Ali, Maura, Arne, Bart and Kelly. I thank you all for the nice time in Maastricht and that I am still everytime welcome when I come back to Maastricht.

Sander, thank you for a lot of nice discussions and for helping me with histological analysis and for introducing me to optical mapping. I think, if I stayed longer in 
Maastricht, I would have continued optical mapping experiments. I am happy to have you as my Co-promotor of my PhD.

Arne, I am very impressed by your way of working, by your organizing talent and by the way you can solve technical problems. When I heard that you also started your own $\mathrm{PhD}$ in Maastricht, I was very happy. You are scientist and technician in one person. This will help you to finish a perfect PhD. I really enjoyed the race bike tours together with you and Bart.

Bart, I am happy that we got to know each other during my time in Maastricht. Not just from the professional but particularly from the personal perspective. I hank you for welcome me several times at your place. We had a nice time and some good bottles of wine and beer together. I will never forget our tandem tour in San Francisco...

Our lab technicians Arne van Hunnik, Marion Kuipers and Anniek Lampert took care for the animals and performed perfect surgery and experiments. Your skill and ideas are essential for the research and experiments done in our group.

During my time in Maastricht, I had the opportunity to get to know Maurits Allessie. Maurit's door was everytime open and everyone was welcome. Maurits, you tought us to retest and question ones own data and to accept ones own failure. Still when we meet at conferences, I enjoy talking and listening to you. Your experience helps us young people to find our way in the knowadays research society. One thing what I learned from you is not to be mainstream but to be different and at the same time authentic.

Prof. Michael Böhm, thank you for beeing the supervisor of my PhD-thesis and supporting my research plans in Homburg/Saar. 
Dear Klaus, without your support and the help of Björn, Thomas and Jenny, this thesis would never have been be possible. Thank you for giving me the oppertunity to perform a lot of experiments in your lab.

Furthermore I would like to thank the assessment committee for reading and evaluating this thesis: Prof. Frits Prinzen, Dr. Klaus Wirth, Prof. Thomas Unger, Prof. Harry Crijns and Prof. Isabelle van Gelder. Your participation in the defense is also very much appreciated.

Last but for sure not least I thank my parents and my brothers Julian and Benedikt and Martina. Thank you for supporting me in all regards during my time in Maastricht and afterwards. I know that you are everytime there and that I can count on you. 


\section{Curriculum Vitae}

26.12.1982

2002

$2002-2009$

2009

02.09.2009

$01.08 .09-30.10 .10$

Since 1.11 .10
Born in Giessen/Germany

Abitur (High School Diploma)

Medicine degree in Mainz, Germany

State examination in medicin (MD)

Conferral of doctorate (Dr. med.)

Research Fellowship

(Cardiovascular Research Institute Maastricht)

Resident of cardiology

(University Homburg/Saar)

\section{Awards:}

2011

2011

2011

2012

2012

2012

2012

2013

2013
Traveller award, Deutschen Gesellschaft für Kardiologie

Young Investigator Award, Deutsche Hochdruckliga

Poster Prize, Deutschen Hochdruckliga

Innovationspreis der Deutschen Hochschulmedizin

Traveller award, European Society of Cardiology,

Young Investigator Award, Deutsche Hochdruckliga

Poster Prize, Deutschen Hochdruckliga

Traveller award, EHRA EUROPACE

Best Poster, ESC-Congress 


\section{Publications}

\section{Journal Articles}

1. Wirth KJ, Brendel J, Steinmeyer K, Linz D, Gögelein H. (2007) In vitro and in vivo effects of the atrial selective antiarrhythmic compound AVE1231.

Journal of Cardiovascular Pharmacology 49:197-206.

2. Linz DK, Afkham F, Itter G, Rütten H, Wirth KJ. (2007) Effect of atrial electrical remodelling on the efficacy of antiarrhythmic drugs: comparison of Amiodarone with IKr- and Ito/IKur-blockade in vivo.

Journal of Cardiovascular Electrophysiology 18:1313-1320.

3. Eckstein J, Maesen B, Linz D, Zeemering S, van Hunnik A, Verheule S, Allessie M, Schotten U. (2010) Time course and mechanisms of endo-epicardial electrical dissociation during atrial fibrillation in the goat.

Cardiovascular Research 89:816-824.

4. Linz D, Schotten U, Neuberger HR, Böhm M, Wirth K. (2011) Negative tracheal pressure during obstructive respiratory events promotes atrial fibrillation by vagal activation.

Heart Rhythm 8:1436-1443.

5. Linz D, Schotten U, Neuberger HR, Böhm M, Wirth K. (2011) Combined blockade of early and late activated atrial potassium currents suppresses atrial fibrillation in a pig model of obstructive apnea.

Heart Rhythm 8:1933-1939.

6. Linz D, Neuberger HR. (2012) Obstructive sleep apnea: The suffocated atrium? Heart Rhythm 9:328-329. 
7. Daniels $A^{*}$, Linz $D^{\star}$, van Bilsen M, Sadowski T, Ruf $S$, Juretschke HP, NeumannHaefelin C, Munts C, van der Vusse G, van Nieuwenhoven F. (2012) Long-term severe diabetes only leads to mild cardiac diastolic dysfunction in Zucker diabetic fatty rats.

\section{European Journal of Heart Failure 14:193-201. (equal contribution*)}

8. Linz D, Mahfoud F, Schotten U, Ukena C, Neuberger HR, Wirth K, Böhm M. (2012) Renal sympathetic denervation suppresses postapneic blood pressure rises and atrial fibrillation in a model for sleep apnea.

Hypertension, 60:172-178.

9. Mahfoud F, Cremers B, Janker J, Link B, Vonend O, Ukena C, Linz D, Schmieder R, Rump LC, Kindermann I, Sobotka PA, Krum H, Scheller B, Schlaich M, Laufs U, Böhm M. (2012) Renal hemodynamics and renal function after catheter-based renal sympathetic denervation in patients with resistant hypertension.

Hypertension 60:419-424.

10. Ruf S, Buning C, Schreuder H, Horstick G, Linz W, Olpp T, Pernerstorfer J, Hiss K, Kroll K, Kannt A, Kohlmann M, Linz D, Hübschle T, Rütten H, Wirth K, Schmidt T, Sadowski T. (2012) Novel B-amino acid derivatives as inhibitors of cathepsin A. Journal of Medicinal Chemistry, 2012;55:7636-7649.

11. Ukena C, Mahfoud F, Kindermann I, Spies A, Linz D, Cremers B, Laufs U, Neuberger HR, Böhm M. (2012) Effects of renal sympathetic denervation on heart rate and atrioventricular conduction in patients with resistant hypertension. International Journal of Cardiology, in press.

12. Linz D, Wirth K. (2012) Intrathoracic pressure oscillations during obstructive apneas disturb ventricular repolarisation.

European Journal of Applied Physiology, 2012;112:4181. 
13. Linz D, Hohl M, Mahfoud F, Reil JC, Linz W, Hübschle T, Juretschke HP, Neumann-Haefelin C, Ruetten H, Böhm M. (2012) Cardiac remodeling and myocardial dysfunction in obese spontaneously hypertensive rats.

Journal of Translational Medicine, 2012;10:187.

14. Linz D. (2012) Atrial fibrillation in obstructive sleep apnea: atrial arrhythmogenic substrate of a different sort.

American Journal of Cardiology, 2012;110:1071.

15. Linz D, Neuberger HR. (2012) Chronic kidney disease and atrial fibrillation. Heart Rhythm, 2012;9:2032-2033.

16. Linz D, Wirth K, Linz W, Heuer HOO, Frick W, Hofmeister A, Heinelt U, Arndt P, Schwahn U, Böhm M, Rütten H. (2012) Antihypertensive and laxative effects by pharmacological inhibition of NHE3-mediated sodium absorption in the gut. Hypertension, 2012;60:1560-1567.

17. Linz D, Mahfoud F, Schotten U, Ukena C, Hohl M, Neuberger HR, Wirth K, Böhm M. (2013) Renal Sympathetic Denervation Provides Ventricular Rate Control But Does Not Prevent Atrial Electrical Remodeling During Atrial Fibrillation. Hypertension, 2013;61:225-231.

18. Eckstein J, Zeemering S, Linz D, Maesen B, Verheule S, van Hunnik A, Crijns H, Allessie MA, Schotten U. (2013) Transmural Conduction is the Predominant Mechanism of Breakthrough during Atrial Fibrillation: Evidence from Simultaneous Endo-epicardial High Density Activation Mapping.

Circulation: Arrhythmia and Electrophysiology, 2013;6:334-341.

19. Wohlfart P, Linz W, Hübschle T, Linz D, Huber J, Hess S, Crowther D, Werner U, Ruetten H. (2013) Cardioprotective effects of lixisenatide in rat myocardial ischemiareperfusion injury studies.

Journal of Translational Medicine. 2013;11:84. 
20. Linz D, Mahfoud F, Schotten U, Ukena C, Neuberger HR, Wirth K, Böhm M. (2013) Effects of Electrical Stimulation of Carotid Baroreflex and Renal Denervation on Atrial Electrophysiology.

Journal of Cardiovascular Electrophysiology. Accepted manuscript online: DOI: 10.1111/jce. 12171 .

21. Linz D, Wirth K, Ukena C, Mahfoud F, Pöss J, Linz B, Böhm M, Neuberger HR. (2013) Renal Denervation Suppresses Ventricular Arrhythmias During Acute Ventricular Ischemia in Pigs.

Heart Rhythm. Accepted manuscript online: DOI:pii: S1547-5271(13)00740-6.

22. Linz D, Hohl M, Nickel A, Mahfoud F, Wagner M, Ewen S, Schotten U, Maack C, Wirth K, Böhm M. (2013) Effect of renal denervation on neurohumoral activation triggering atrial fibrillation in obstructive sleep apnea.

Hypertension. 2013. Accepted.

23. Linz D, Ukena C, Neuberger HR. (2013) Treatment of obstructive sleep apnea improves metabolic conditions and prevents initiation of antiarrhythmic therapy in a patient with atrial fibrillation. Europace. 2013. Accepted.

\section{Reviews:}

1. Linz D, Schmidt J, Zimmermann S, Dauer M, Lammert F, Böhm M. (2011) Kardiovaskuläre Erkrankungen: Folgen für den Gastrointestinaltrakt.

Der Gastroenterologe, $2011 ; 6: 292-299$.

2. Mahfoud F, Linz D, Böhm M. (2013) Heart and kidneys: Renal denervation as therapy for hypertension.

Herz, 2013;38:67-78. 
3. Ruf S, Buning C, Schreuder H, Linz W, Hübschle T, Linz D, Ruetten H, Wirth K, Sadowski T. (2013) Inhibition of CatA: an emerging strategy for the treatment of heart failure.

Future Medicinal Chemistry, 2013;5:399-409.

4. Ewen S, Ukena C, Linz D, Schmieder RE, Böhm M, Mahfoud F. (2013) The Sympathetic Nervous System in Chronic Kidney Disease.

Curr Hypertens Rep. 2013;15:370-376.

5. Ukena C, Mahfoud F, Linz D, Böhm M, Neuberger H-R. (2013) Potential role of renal sympathetic denervation for the treatment of cardiac arrhythmias.

Eurolntervention, 2013;9: Suppl R110-R116.

6. Mahfoud F, Ewen S, Ukena C, Linz D, Sobotka P, Cremers B, Böhm M. (2013) Expanding the indication spectrum: renal denervation in diabetes.

Eurolntervention, 2013;9: Suppl R117-R121.

7. Böhm M, Ewen S, Linz D, Reil J-C, Schirmer S, Ukena C, Mahfoud F. (2013) Therapeutic potential of renal sympathetic denervation in patients with chronic heart failure.

Eurolntervention, 2013;9: Suppl R122-R126.

8. Böhm M, Linz D, Urban D, Mahfoud F, Ukena C. (2013) Renal sympathetic denervation: applications in hypertension and beyond.

Nat Rev Cardiol, 2013;10:465-476. 